University of Wollongong

Research Online

Faculty of Science - Papers (Archive)

Faculty of Science, Medicine and Health

January 2009

\title{
In situ U-Pb, $\mathrm{O}$ and $\mathrm{Hf}$ isotopic compositions of zircon and olivine from Eoarchaean rocks, West Greenland: new insights to making old crust
}

Joe Hiess

NERC Isotope Geosciences Laboratory

Vickie C. Bennett

Australian National University

Allen P. Nutman

University of Wollongong, anutman@uow.edu.au

Ian S. Williams

Follow this and additional works at: https://ro.uow.edu.au/scipapers

Part of the Life Sciences Commons, Physical Sciences and Mathematics Commons, and the Social and Behavioral Sciences Commons

\section{Recommended Citation}

Hiess, Joe; Bennett, Vickie C.; Nutman, Allen P.; and Williams, lan S.: In situ U-Pb, O and Hf isotopic compositions of zircon and olivine from Eoarchaean rocks, West Greenland: new insights to making old crust 2009, 4489-4516.

https://ro.uow.edu.au/scipapers/985

Research Online is the open access institutional repository for the University of Wollongong. For further information contact the UOW Library: research-pubs@uow.edu.au 


\title{
In situ U-Pb, $\mathrm{O}$ and $\mathrm{Hf}$ isotopic compositions of zircon and olivine from Eoarchaean rocks, West Greenland: new insights to making old crust
}

\begin{abstract}
The sources and petrogenetic processes that generated some of the Earth's oldest continental crust have been more tightly constrained via an integrated, in situ (U-Pb, $\mathrm{O}$ and $\mathrm{Hf}$ ) isotopic approach. The minerals analysed were representative zircon from four Eoarchaean TTG tonalites and two felsic volcanic rocks, and olivine from one harzburgite/dunite of the Itsaq Gneiss Complex (IGC), southern West Greenland. The samples were carefully chosen from localities with least migmatisation, metasomatism and strain. Zircon was thoroughly characterized prior to analysis using cathodoluminescence, scanning electron, reflected and transmitted light imaging. The zircon from all but one sample showed only minor post-magmatic recrystallisation. (207) Pb/(106) Pb dating of oscillatory-zoned zircon using SHRIMP RG $(n=142)$ indicates derivation of the felsic igneous rocks from different batches of magma at 3.88, 3.85, 3.81, 3.80 and 3.69 $\mathrm{Ga}$. Analyses of (18)0/(16)O compositions of olivine from a harzburgite/dunite $(n=8)$ using SHRIMP II in multi-collector mode, indicate that the oxygen isotopic composition of this sample of Eoarchaean mantle $($ delta $(18) \mathrm{O}(\mathrm{OI})=6.0+/-0.4$ parts per thousand) was slightly enriched in (18)0, but not significantly different from that of the modern mantle. Zircon delta(18)0 measurements from the six felsic rocks $(\mathrm{n}=$ 93) record mean or weighted mean compositions ranging from $4.9+/-0.7$ parts per thousand to $5.1+/-$ 0.4 parts per thousand, with recrystallised domains showing no indication of oxygen isotopic exchange during younger tectonothermal events. delta(18)O(Zr) compositions indicate that the primary magmas were largely in equilibrium with the mantle or mantle-derived melts generated at similar high temperatures, while calculated tonalite delta(18)O(WR) compositions (6.7-6.9 parts per thousand) resemble those of modern adakites. LA-MC-ICPMS zircon $(176) \mathrm{Hf} /(177) \mathrm{Hf}$ analyses were obtained from six samples $(n=122)$, Five samples record weighted mean initial epsilon $(\mathrm{Hf})$ compositions ranging from to $0.5+/-0.6$ to $-0.1+/-0.7$ (calculated using lambda(176) Lu $=1.867 \times 10(-11) \mathrm{yr}(-1)$ ), while one sample records a composition of $1.3+/-0.7$, indicating the magmas were generated from a reservoir with a time averaged, near chondritic Lu/Hf. The derivation of TTG magmas from a chondritic Lu/Hf source implies either that there was not voluminous continental crustal growth nor major mantle differentiation leading to $\mathrm{Lu} / \mathrm{Hf}$ fractionation during the Hadean or Eoarchaean, or alternatively that rapid recycling of an early formed crust allowed the early mantle to maintain a chondritic Lu/Hf. Previous studies have demonstrated that ancient TTG rocks were mostly produced by dehydration melting of mafic rocks within the stability field of garnet, probably in flatly-subducted or buried oceanic crust. The oxygen isotopic signatures measured here at high spatial resolution allow the source materials to be better defined. Melting of a mixed mafic source consisting of similar to $80 \%$ unaltered gabbro (delta(18)O(WR) $=5.5 \%$.) with similar to $20 \%$ hydrothermally altered gabbro/basalt (delta(18)O(WR) $=4.0$ parts per thousand) would produce tonalite magmas within the average compositional range observed. (18)0-enriched components such as altered shallow basaltic oceanic crust and pelagic or continental sediments were not present in the sources of these TTG melts. The absence of high (18)0 signatures may indicate either the rarity of low temperature altered sediments, or their effective removal from the down-going slab. (C) 2009 Elsevier Ltd. All rights reserved.
\end{abstract}

\section{Keywords}

olivine, zircon, compositions, isotopic, hf, o, pb, u, situ, crust, making, old, insights, greenland, west, rocks, eoarchaean, GeoQUEST

\section{Disciplines}

Life Sciences | Physical Sciences and Mathematics | Social and Behavioral Sciences 


\section{Publication Details}

Hiess, J., Bennett, V. C., Nutman, A. P. \& Williams, I. S. (2009). In situ U-Pb, O and Hf isotopic compositions of zircon and olivine from Eoarchaean rocks, West Greenland: new insights to making old crust.

Geochimica et Cosmochimica Acta, 73 (15), 4489-4516. 


\title{
In situ $\mathrm{U}-\mathrm{Pb}, \mathrm{O}$ and $\mathrm{Hf}$ isotopic compositions of zircon and olivine from Eoarchaean rocks, West Greenland: New insights to making old crust
}

\author{
Joe Hiess $^{\mathrm{a}, *}$, Vickie C. Bennett ${ }^{\mathrm{a}}$, Allen P. Nutman ${ }^{\mathrm{b}}$, Ian S. Williams ${ }^{\text {a }}$ \\ ${ }^{a}$ Research School of Earth Sciences, Australian National University, Canberra, ACT 0200, Australia \\ ${ }^{\mathrm{b}}$ Institute of Geology, Chinese Academy of Geological Sciences, 26 Baiwanzhuang Road, Beijing 100037, China
}

Received 29 July 2008; accepted in revised form 17 April 2009; available online 3 May 2009

\begin{abstract}
The sources and petrogenetic processes that generated some of the Earth's oldest continental crust have been more tightly constrained via an integrated, in situ ( $\mathrm{U}-\mathrm{Pb}, \mathrm{O}$ and $\mathrm{Hf}$ ) isotopic approach. The minerals analysed were representative zircon from four Eoarchaean TTG tonalites and two felsic volcanic rocks, and olivine from one harzburgite/dunite of the Itsaq Gneiss Complex (IGC), southern West Greenland. The samples were carefully chosen from localities with least migmatisation, metasomatism and strain. Zircon was thoroughly characterized prior to analysis using cathodoluminescence, scanning electron, reflected and transmitted light imaging. The zircon from all but one sample showed only minor post-magmatic recrystallisation. ${ }^{207} \mathrm{~Pb} /{ }^{206} \mathrm{~Pb}$ dating of oscillatory-zoned zircon using SHRIMP RG $(n=142)$ indicates derivation of the felsic igneous rocks from different batches of magma at 3.88, 3.85, 3.81, 3.80 and $3.69 \mathrm{Ga}$.

Analyses of ${ }^{18} \mathrm{O} /{ }^{16} \mathrm{O}$ compositions of olivine from a harzburgite/dunite $(n=8)$ using SHRIMP II in multi-collector mode, indicate that the oxygen isotopic composition of this sample of Eoarchaean mantle $\left(\delta^{18} \mathrm{O}_{\mathrm{Ol}}=6.0 \pm 0.4 \%\right.$ was slightly enriched in ${ }^{18} \mathrm{O}$, but not significantly different from that of the modern mantle. Zircon $\delta^{18} \mathrm{O}$ measurements from the six felsic rocks $(n=93)$ record mean or weighted mean compositions ranging from $4.9 \pm 0.7 \%$ to $5.1 \pm 0.4 \%$, with recrystallised domains showing no indication of oxygen isotopic exchange during younger tectonothermal events. $\delta^{18} \mathrm{O}_{\mathrm{Zr}}$ compositions indicate that the primary magmas were largely in equilibrium with the mantle or mantle-derived melts generated at similar high temperatures, while calculated tonalite $\delta^{18} \mathrm{O}_{\mathrm{WR}}$ compositions $(6.7-6.9 \%$ ) resemble those of modern adakites.

LA-MC-ICPMS zircon ${ }^{176} \mathrm{Hf} /{ }^{177} \mathrm{Hf}$ analyses were obtained from six samples $(n=122)$. Five samples record weighted mean initial $\varepsilon_{\mathrm{Hf}}$ compositions ranging from to $0.5 \pm 0.6$ to $-0.1 \pm 0.7$ (calculated using $\lambda^{176} \mathrm{Lu}=1.867 \times 10^{-11} \mathrm{yr}^{-1}$ ), while one sample records a composition of $1.3 \pm 0.7$, indicating the magmas were generated from a reservoir with a time averaged, near chondritic Lu/Hf. The derivation of TTG magmas from a chondritic Lu/Hf source implies either that there was not voluminous continental crustal growth nor major mantle differentiation leading to Lu/Hf fractionation during the Hadean or Eoarchaean, or alternatively that rapid recycling of an early formed crust allowed the early mantle to maintain a chondritic $\mathrm{Lu} / \mathrm{Hf}$.

Previous studies have demonstrated that ancient TTG rocks were mostly produced by dehydration melting of mafic rocks within the stability field of garnet, probably in flatly-subducted or buried oceanic crust. The oxygen isotopic signatures measured here at high spatial resolution allow the source materials to be better defined. Melting of a mixed mafic source consisting of $\sim 80 \%$ unaltered gabbro $\left(\delta^{18} \mathrm{O}_{\mathrm{WR}}=5.5 \%\right.$ ) with $\sim 20 \%$ hydrothermally altered gabbro/basalt $\left(\delta^{18} \mathrm{O}_{\mathrm{WR}}=4.0 \%\right.$ would produce tonalite magmas within the average compositional range observed. ${ }^{18} \mathrm{O}$-enriched components such as altered shallow basaltic oceanic crust and pelagic or continental sediments were not present in the sources of these TTG melts. The absence
\end{abstract}

\footnotetext{
* Corresponding author. Present address: Korea Basic Science Institute, 804-1 Yangcheong-ri, Ochang, Cheongwon-gun, Chungbuk 363883, Republic of Korea. Fax: +82432405179.

E-mail address: joehiess@kbsi.re.kr (J. Hiess).
} 
of high ${ }^{18} \mathrm{O}$ signatures may indicate either the rarity of low temperature altered sediments, or their effective removal from the down-going slab.

(C) 2009 Elsevier Ltd. All rights reserved.

\section{INTRODUCTION}

Archaean tonalite-trondhjemite-granodiorite (TTG) granitoids (Jahn et al., 1981) comprise $\sim 90 \%$ of the juvenile continental crust generated between 4.0 and $2.5 \mathrm{Ga}$ (Martin et al., 1983). It is now widely accepted that the silica and sodium rich, heavy rare earth element (HREE) depleted tonalitic magmas of TTG suites were generated by partial melting of a hydrated, low-K basaltic crust at pressures sufficient to stabilize garnet, i.e. transformed to eclogite or garnet amphibolite (e.g. Barker and Arth, 1976; Martin, 1986, 1987; Drummond and Defant, 1990; Rapp et al., 1991; Rapp and Watson, 1995; Rapp et al., 2003; Martin et al., 2005). Condie (1981) applied the concept of modern plate tectonics in proposing that the basaltic source of these ancient magmas was subducted oceanic crust. Contrasting mechanisms for the formation of TTG-like magmas have also been suggested, for example partially melting of the basal section of a magmatically thickened, mafic arc-crust (Atherton and Petford, 1993) or partial melting of a delaminated mafic lower-crustal underplate outside an arc setting (Xu et al., 2002). Both scenarios are complicated by the need to identify mechanisms to hydrate the basaltic crust, prior to partial melting within the stability field of garnet. Regardless, most models for the genesis of TTG magmas contrast with those for producing the most common types of magmas in modern arcs (e.g. Kamber et al., 2002), where hydrous fluxing off a subducting slab provides fluids for melting in the overlying mantle wedge (Gill, 1981). Archaean TTG magmas, however, have some affinity to modern adakites, a less common variety of modern arc magmas. It has been suggested that these magmas are produced by melting hot oceanic crust transformed to garnet amphibolite or eclogite during shallow subduction (Kay, 1978; Martin, 1986; Defant and Drummond, 1990; Martin, 1999).

In order to better constrain the petrogenetic processes operating during the formation of early continents as represented by Eoarchaean tonalitic magmas, we have undertaken U-Pb, O (SHRIMP) and Hf (LA-MC-ICPMS) in situ isotopic analysis of well characterized zircon from four well-preserved 3.88-3.69 Ga tonalites and two $3.8 \mathrm{Ga}$ felsic volcanic rocks, and olivine from one sample of $>3.8 \mathrm{Ga}$ mantle harzburgite/dunite from the Itsaq Gneiss Complex (IGC), southern West Greenland (Nutman et al., 1996). These rocks have undergone either amphibolite or granulite facies metamorphism (e.g. Griffin et al., 1980; Friend and Nutman, 2005) and are therefore mostly recrystallised since their igneous emplacement, except for relict oscillatory-zoned domains within zircon and rare relict olivine such as in the one ultramafic sample studied here (Friend et al., 2002). Combining imaging with high spatial resolution analytical techniques, we have tried to analyse the best-preserved domains within these relict igneous zircon and mantle olivine crystals in order to determine the isotopic composition of the magmas from which the ancient TTG rocks crystallised.

If shallow crustal-level contaminants, with their distinctly non mantle-like ${ }^{18} \mathrm{O} /{ }^{16} \mathrm{O}$ ratios were present in the source region of Eoarchaean tonalite magmas at $>1000{ }^{\circ} \mathrm{C}$ and $>20$ kbar (Rapp and Watson, 1995; Moyen and Stevens, 2006), it would imply recycling of crustal materials back into the mantle during Eoarchaean convergent plate-boundary magmatism. This would have important implications for early geodynamics, the distribution of heat-producing elements and several geochemical cycles. Using high spatial resolution $\mathrm{U}-\mathrm{Pb}, \mathrm{O}$ and $\mathrm{Hf}$ isotopic analysis of igneous zircon from Eoarchaean tonalites we have tested whether such recycling occurred, placed firmer constraints on the petrogenesis of Eoarchaean felsic magmas, and thereby have increased our understanding of the broad processes that operated early in the history of the Earth.

\section{ZIRCON AS A GEOCHEMICAL TOOL TO UNRAVEL ANCIENT PROCESSES}

Zircon is an ideal phase for Hf isotopic tracing in ancient rocks (Kinny and Maas, 2003). Typically zircon contains tens of ppm of Lu but $\sim 1 \%$ Hf. It is thereby the major host for Hf in granitoids (Hoskin and Schaltegger, 2003). Most importantly, very low $\mathrm{Lu} / \mathrm{Hf}$ ratios result in small corrections for the in-growth of radiogenic ${ }^{176} \mathrm{Hf}$ from ${ }^{176} \mathrm{Lu}$ decay, and $\mathrm{Hf}$ is generally immobile unless the zircon is completely destroyed (Pettingill and Patchett, 1981). Consequently, $\mathrm{Hf}$ in zircon can be used as a robust isotopic tracer to track terrestrial crustal growth and mantle evolution (Patchett et al., 1981).

The initially uniform, chondritic Lu/Hf of the Earth has become progressively modified by silicate differentiation over time, with Hf-enriched crustal reservoirs ( $\mathrm{Lu} / \mathrm{Hf}<$ chondrites) evolving to negative measured $\varepsilon_{\mathrm{Hf}}$ compositions, where $\quad \varepsilon_{\mathrm{Hf}(\mathrm{T})}=\left({ }^{176} \mathrm{Hf} /{ }^{177} \mathrm{Hf}_{\text {sample }(\mathrm{T})} /{ }^{176} \mathrm{Hf} /{ }^{177} \mathrm{Hf}_{\mathrm{CHUR}(\mathrm{T})}\right)-$ $1 \times 10^{4}$. Extraction of continental crust has subsequently resulted in the formation of a complementary mantle reservoir that is depleted in hafnium ( $\mathrm{Lu} / \mathrm{Hf}>$ chondrites), leading to a modern depleted mantle $\varepsilon_{\mathrm{Hf}}$ of ca. +16 (e.g. Chauvel and Blichert-Toft, 2001). The time-corrected ${ }^{176} \mathrm{Hf} /{ }^{177} \mathrm{Hf}$ composition of an igneous zircon (established using its $\mathrm{U}-\mathrm{Pb}$ age) can be used to distinguish between derivation of the host magma from an undifferentiated chondritic reservoir (CHUR) in terms of $\mathrm{Lu} / \mathrm{Hf}$, a depleted mantle reservoir that has experienced previous crustal extraction, a reservoir consisting of older crustal components, or some mixture of these (e.g. Amelin et al., 1999, 2000).

Oxygen isotope ratios in pristine igneous zircon are sensitive indicators of magma contamination by lithologies that have been subject to water-rock interaction (Valley, 2003). Crustal materials that have been subject to 
low-temperature or moderate-temperature $\left(0-100^{\circ} \mathrm{C}\right)$ water interaction, such as weathered continental crust, oceanic sediments, or altered shallow oceanic crust, carry a distinctly ${ }^{18} \mathrm{O}$-enriched signature (Kolodny and Epstein, 1976; Alt et al., 1986). In contrast, ${ }^{18} \mathrm{O}$-depleted signatures result from high-temperature $\left(>100^{\circ} \mathrm{C}\right)$ or very-low-temperature $\left(<0{ }^{\circ} \mathrm{C}\right)$ water interaction, and occur in materials such as hydrothermally altered deep oceanic crust, or rocks altered by cold meteoric water (Gregory and Taylor, 1981; Criss and Taylor, 1986). Progressive addition of ${ }^{18} \mathrm{O}$-enriched or ${ }^{18} \mathrm{O}$-depleted contaminants to a primitive igneous system can result in the divergence of ${ }^{18} \mathrm{O} /{ }^{16} \mathrm{O}$ ratios from that of mantle-derived melts, or magmas equilibrated at similar temperatures (Eiler, 2001; Valley et al., 2005).

Taken together, the $\mathrm{Hf}$ and $\mathrm{O}$ isotopic signatures of precisely dated zoned zircon can reveal both source environments and near surface fluid history of magma protoliths (e.g. Kemp et al., 2007; Bolhar et al., 2008). Not only do they provide critical new evidence for the origin of TTG magmas, the integrated application of these relatively new in situ techniques to zircon extracted from previously well studied Eoarchaean rocks also allows for examination of the behavior of each isotope system within the zircon population over extensive crustal residence times $(>3.7 \mathrm{Ga})$ and can be used to reveal the post-crystallisation history of overprinting metamorphic events and migmatisation. The range of $\mathrm{U}-\mathrm{Pb}, \mathrm{O}$ and $\mathrm{Hf}$ isotopic compositions for multiple zircon grains derived from a single rock is also of relevance when interpreting the significance of complex variations present in detrital zircon populations (e.g. Amelin et al., 1999; Harrison et al., 2005; Kemp et al., 2006).

\section{SAMPLES AND THEIR GEOLOGICAL SETTING}

\subsection{The Itsaq Gneiss Complex (IGC)}

The zircon and olivine samples examined for this study were extracted from meta-tonalites, felsic meta-volcanic rocks and one ultramafic rock of the IGC (Nutman et al., 1996, 2007a), southern West Greenland. The complex extends over $\sim 3000 \mathrm{~km}^{2}$ in the Nuuk region, and offers rare examples of well-preserved Eoarchaean rocks from granitic to ultramafic composition that provide direct information on early Earth processes (e.g. Nutman et al., 1999; Whitehouse et al., 1999; Bennett et al., 2002; Polat et al., 2002; Whitehouse and Kamber, 2002; Polat and Hofmann, 2003; Frei and Polat, 2007; Bennett et al. 2007). The IGC is dominated by quartzo-feldspathic rocks (largely TTG tonalites) that were intruded into ultramafic, mafic and sedimentary rocks during several periods of igneous activity between 〜3.88 and 3.62 Ga (Nutman et al., 1999, 2000, 2007b).

The IGC has been severely overprinted by a complex and protracted series of orogenic events with associated amphibolite to granulite facies metamorphism. Eoarchaean events $(\sim 3.65-3.54 \mathrm{Ga})$ included in situ partial melting of the tonalites in the south of the complex, the generation of crustally derived granites (sensu stricto), and the tectonic intercalation of unrelated rocks along major mylonites reflecting crustal shortening at convergent plate margins (Nutman and Bridgwater, 1986; Nutman et al., 2002;
Friend and Nutman, 2005; Nutman et al., 2007a). Consequently, primary igneous textures and intrusive relationships were mostly obliterated during heterogeneous ductile deformation and replaced by tectonic fabrics and amphibolite facies mineral assemblages (Nutman et al., 2000). Subsequent events include the intrusion of Mesoarchaean dolerite dikes (McGregor, 1973) and polyphase regional ductile deformation during and following terrane amalgamation in the Neoarchaean (Friend et al., 1987; Nutman and Friend, 2007).

Fortuitously, extremely rare, low strain domains survive locally in the amphibolite facies Eoarchaean orthogneisses where metamorphic grade failed to reach migmitisation and overprinting deformation is minimal (Nutman et al., 1999, 2000, 2007b). These are mostly in the northern part of the complex, near the Isua supracrustal belt. At such localities rare single-phase tonalite and rarer ultramafic rocks can be sampled that are devoid of partial melt (G01/113, G97/18, 248228, G93/42), or contain only small amounts of such material (G01/36), and volcanic rocks can be collected where the amount of alteration products can still be observed $(248202,248203)$. This sampling method allows for the most definitive geochronological and geochemical characterization of the rock protoliths, essential for the clearest interpretation of petrogenetic processes operating during the formation of the Eoarchaean crust (Nutman et al., 1999; Bennett et al., 2002; Friend et al., 2002; Polat and Hofmann, 2003; this study).

\subsection{Tonalite samples G01/36, G01/113, G97/18 and 248228}

The four meta-tonalite samples analysed in this study have geochemical characteristics typical of Archaean TTG suites. They are metaluminous, rich in $\mathrm{SiO}_{2}$ and $\mathrm{Al}_{2} \mathrm{O}_{3}$, yet poor in $\mathrm{MgO}$ and $\mathrm{K}_{2} \mathrm{O}$. They show LREE enrichment, HREE depletion, small positive Eu anomalies and high $\mathrm{Sr} /$ Y. Major and trace element data are presented in Nutman et al. (1996, 1999, 2007b). Bulk compositions can be explained by $30-40 \%$ melting of a hydrated mafic source under pressures sufficient for stabilization of residual garnet, clinopyroxene and rutile (eclogite), with variable degrees of plagioclase fractionation superimposed during emplacement (Nutman et al., 1999).

The Eoarchaean IGC tonalites have low initial ${ }^{87} \mathrm{Sr} /{ }^{86} \mathrm{Sr}$ (e.g. Moorbath et al., 1972), positive initial $\varepsilon_{143 \mathrm{Nd}}$ (e.g. Bennett et al., 1993), and positive ${ }^{142} \mathrm{Nd}$ anomalies relative to modern rocks due to the early decay of now-extinct ${ }^{146} \mathrm{Sm}$ (Caro et al., 2003, 2006; Bennett et al., 2007). These signatures indicate genesis of the tonalites as additions of juvenile crust, with no evidence for major incorporation of much older pre-existing crustal components. The first oxygen isotopic investigation of IGC rocks was made by Baadsgaard et al. (1986), who presented a mean whole rock $\delta^{18} \mathrm{O}_{\mathrm{WR}}$ of $7.6 \pm 0.3 \%$ for 15 fresh $\sim 3.7 \mathrm{Ga}$ grey (tonalitic) gneisses from the Isukasia area. Cates and Mojzsis (2006) reported whole rock $\left(\delta^{18} \mathrm{O}_{\mathrm{WR}}=7.4 \pm 0.1 \%\right.$ ) and SIMS zircon $\left(\delta^{18} \mathrm{O}_{\mathrm{Zr}}=\right.$ from $6.9 \%$ "core" to $8.5 \%$ "rim") compositions for a single IGC banded tonalitic gneiss sample GR0083 (3617 $\pm 34 \mathrm{Ma})$. The ${ }^{18} \mathrm{O}$-enriched, concordant, oscillatory zoned zircon overgrowths in this sample were 
interpreted as metamorphic zircon grown in equilibrium with crustal fluids (Cates and Mojzsis, 2006).

Igneous zircon populations from the tonalites studied here have been well characterized in previous studies (Baadsgaard, 1983; Nutman et al., 1996, 1999, 2000; Honda et al., 2003; Nutman et al., 2007a,b; Hiess et al., 2008; Nutman and Hiess, 2009). Zircon exhibiting oscillatory zoned growth textures has moderate $\mathrm{Th} / \mathrm{U} \quad(>0.30)$ and ${ }^{207} \mathrm{~Pb} /{ }^{206} \mathrm{~Pb}$ ages corresponding to separate intrusive events at $\sim 3.88,3.85,3.81$ and $3.69 \mathrm{Ga}$. Zircon recrystallisation domains, Pb-loss domains and metamorphic overgrowths have lower $\mathrm{Th} / \mathrm{U}(<0.30)$ and ${ }^{207} \mathrm{~Pb} /{ }^{206} \mathrm{~Pb}$ ages. The ages range from those measured on oscillatory zoned domains to $\sim 3.54 \mathrm{Ga}$, by which time younger Eoarchaean metamorphic events had finished (Friend and Nutman, 2005). Even less common are rims formed later at $\sim 2.72 \mathrm{Ga}$. In all samples except for G01/36 these modifications to the igneous oscillatory zoned zircon are minimal. Published details relevant to each tonalite sample used in this study are summarized in Electronic Annex EA-1.

\subsection{Felsic volcanic samples 248202 and 248203}

Samples 248202 and 248203, were collected from a unit of felsic schist that crops out throughout the length of the southern, $\sim 3.8 \mathrm{Ga}$ section of the Isua supracrustal belt (Fig. 1; $65^{\circ} 05.83^{\prime} \mathrm{N} 50^{\circ} 00.00^{\prime} \mathrm{W}$ ). This unit commonly shows strong enrichment in $\mathrm{K}_{2} \mathrm{O}$, with probable leaching of $\mathrm{Na}_{2} \mathrm{O}$, and locally high carbonate content (Nutman et al., 1984). Multigrain isotope dilution $\mathrm{U}-\mathrm{Pb}$ dating of zircon by Baadsgaard et al. (1984) was followed by ion microprobe analyses by Compston et al. (1986) to provide a crystallisation age of $3807 \pm 2 \mathrm{Ma}$. The oscillatory-zoned igneous zircon grains from these rocks commonly have tubular structures (Fig. 2). Detailed SEM imaging shows that these are voids within the zircon, not inclusions of other minerals. The voids demonstrate that these zircons grew from a melt from which a fluid phase was exsolving. Therefore, the protoliths of these rocks cannot have been deep-seated plutonic rocks, but must have been hypabyssal intrusive or volcanic rocks. These rocks were included in this study to investigate

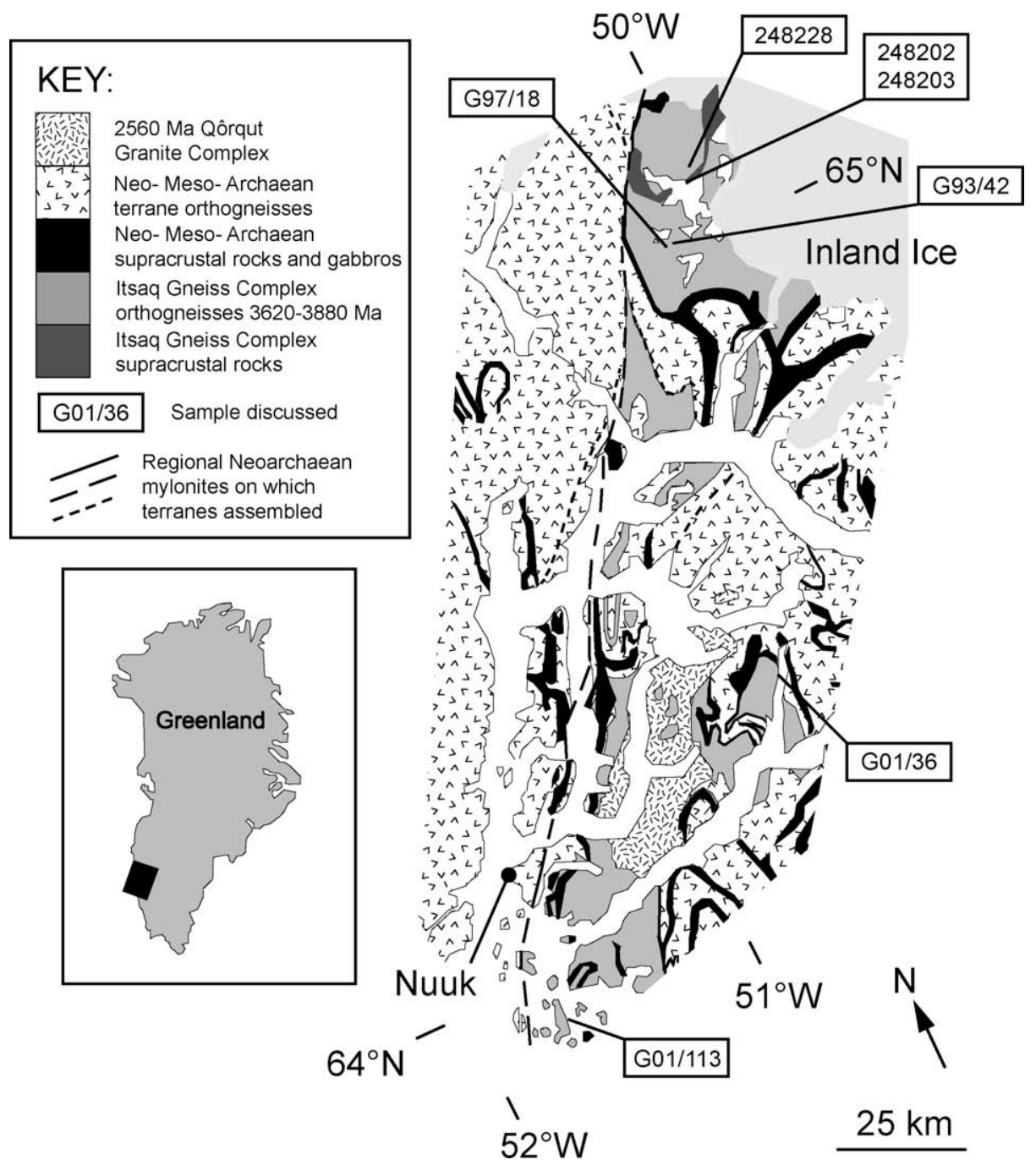

Fig. 1. Sketch geological map of Nuuk region, southern West Greenland with major lithological units and samples discussed. Adapted after Nutman et al. (2007b). 


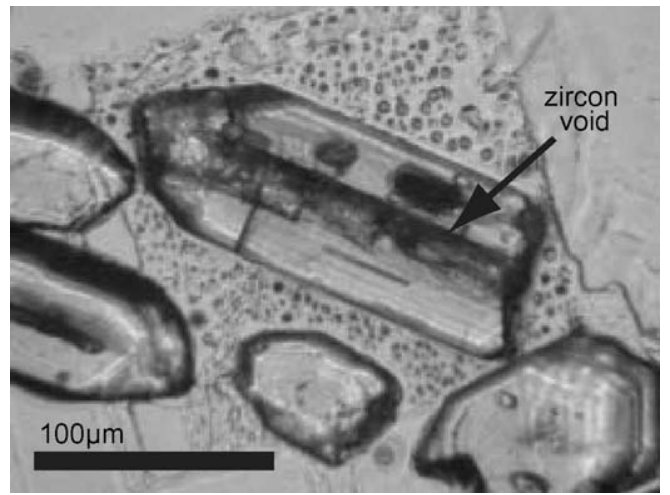

Fig. 2. Transmitted light image of zircon from 248202 with elongate tubular voids.

the possible existence of contrasting $\mathrm{O}$ and $\mathrm{Hf}$ isotopic compositions in shallower, coeval $\sim 3.8 \mathrm{Ga}$ magmatic systems which might be more heavily influenced by incorporation of near-surface altered materials.

\subsection{Ultramafic Sample G93/42}

Dunite and harzburgite occur in ultramafic schist bodies (up to $2 \mathrm{~km} \times 200 \mathrm{~m}$ ) that are a very minor $(\ll 1 \%$ ) component of the IGC. These rocks have major element compositions that conform to the mantle differentiation trend, so are interpreted as slivers of variably-depleted upper mantle caught up in the crust during accretion (Nutman et al., 1996; Friend et al., 2002). In low strain domains these ultramafic enclaves are crosscut by quartzo-feldspathic gneisses that have been dated by SHRIMP $\mathrm{U}-\mathrm{Pb}$ method at ca. $3.8 \mathrm{Ga}$ providing a minimum age of the ultramafic rocks (Nutman et al., 1996; Friend et al., 2002). The ultramafic rocks are typically hydrated, carbonated or serpentinised due to regional metamorphic events throughout the Archaean (Dymek et al., 1988). Some larger bodies, however, contain rare cores that are largely anhydrous, with minimal alteration or hydrous phases, providing the best targets for investigations of early mantle geochemistry (e.g. Bennett et al. 2002; Friend et al. 2002; this study). The olivine from sample G93/42 used in this study is sourced from a rare, least-altered, massive harzburgite/dunite body that is enclosed within TTG gneisses south of Isua (Fig. $1 ; 65^{\circ} 00.58^{\prime} \mathrm{N} 50^{\circ} 12.42^{\prime} \mathrm{W}$ ).

Harzburgite-dunite sample G93/42 is dominated by medium to fine grained, unzoned magnesian olivine $(\mathrm{Mg \#}=89.4)$ with abundant $120^{\circ}$ grain boundary junctions. Other phases include magnesian orthopyroxene $\left(\mathrm{En}_{89}\right)$, green or brown $\mathrm{Al}$ and $\mathrm{Cr}$ rich spinel $(\mathrm{Cr} \#=18.2)$ and rare clinopyroxene or hydrous phases (Friend et al., 2002). Representative petrographic images of G93/42 are located in Electronic Annex EA-2.

Mineral and whole rock major and REE compositions indicate that these rocks resemble low pressure $(<15 \mathrm{kbar})$ mantle restite, following small degrees $(<10 \%)$ of melt extraction (Friend et al., 2002). Such compositions are typical of modern abyssal spinel bearing peridotites, making such samples the least altered direct sample of the Eoarchaean upper mantle yet identified (Bennett et al., 2002; Friend et al., 2002). On the basis of a range of geochemical criteria, including primitive ${ }^{187} \mathrm{Os} /{ }^{188} \mathrm{Os}$ compositions from olivine and spinel separates (Bennett et al., 2002), G93/42 was identified by

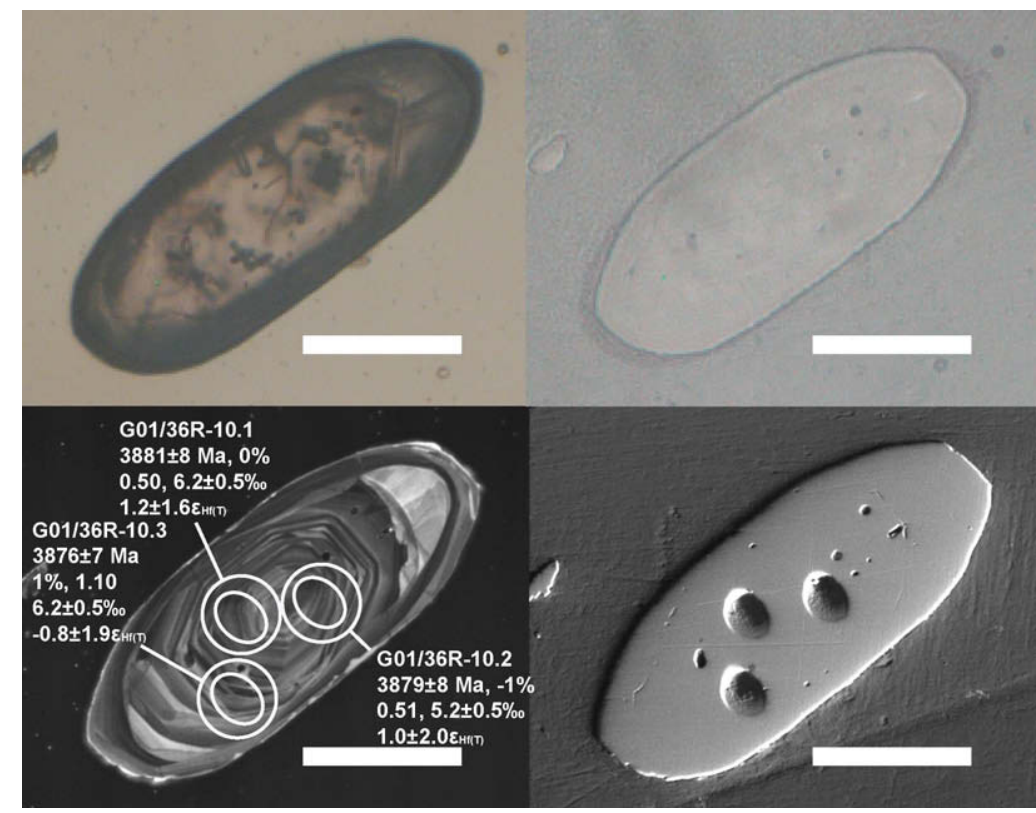

Fig. 3. Tonalite zircon G01/36R-10 as imaged (clockwise from top left) by: transmitted light, reflected light, secondary electrons (SE) and cathodoluminesence (CL). Scale bar is $100 \mu \mathrm{m}$. CL image indicates location of coinciding ${ }^{207} \mathrm{~Pb} /{ }^{206} \mathrm{~Pb}$ age, discordance, Th/U and $\delta^{18} \mathrm{O}$ determinations (separated by mount polishing) with $30 \mu \mathrm{m}$ diameter elliptical spots. Both analyses performed on precisely the same domain of oscillatory zoned zircon. SE image following ${ }^{18} \mathrm{O} /{ }^{16} \mathrm{O}$ measurement indicates the analysis was performed on pristine zircon, free of cracks or inclusions. ${ }^{176} \mathrm{Hf} /{ }^{177} \mathrm{Hf}$ analysis performed on larger, $47 \mu \mathrm{m}$ diameter circular spots from essentially the same crystal growth domain. 
Rollinson (2007) as one of only two samples worldwide (both collected from the same locality) that fit his most stringent criteria for identifying Eoarchaean mantle samples.

\section{RESULTS}

Analytical methods are described at the end of the paper. A summary of zircon and olivine data consisting of $142 \mathrm{U}-$ $\mathrm{Pb}, 101 \mathrm{O}^{18} / \mathrm{O}^{16}$ and $122{ }^{176} \mathrm{Hf} /{ }^{177} \mathrm{Hf}$ analyses is presented in Table 1. Analyses used for weighted mean or mean calculations and the summary statistics for each sample are marked in bold. Details of each O and Hf sample analysis are given in Electronic Annexes EA-6 and EA-7. Representative $\mathrm{CL}$ images with zircon analysis locations are given for G01/36 in Electronic Annex EA-8, and for G01/113 and $\mathrm{G} 97 / 18$ in Fig. 5. Terra-Wasserburg ${ }^{238} \mathrm{U} /{ }^{206} \mathrm{~Pb}-{ }^{207} \mathrm{~Pb} /{ }^{206} \mathrm{~Pb}$ diagrams, plots of $\delta^{18} \mathrm{O}$ and $\varepsilon_{\mathrm{Hf}(\mathrm{T})}$ compositions against corresponding ${ }^{207} \mathrm{~Pb} /{ }^{206} \mathrm{~Pb}$ crystallisation age for each zircon analysis, and sample weighted means or means are illustrated in Fig. 6. The field for mantle zircon $\left(\delta^{18} \mathrm{O}=5.3 \pm 0.3 \%\right)$ is the composition of zircon derived from the mantle or melts equilibrated at similar temperatures (Valley et al., 1998). The field for Archaean and Hadean "supracrustal zircon" $\left(\delta^{18} \mathrm{O}=6.5-7.5 \%\right.$ ) reflects the composition of zircon from igneous protoliths whose source materials were altered by low temperature interaction with liquid water near Earth's surface (Cavosie et al., 2005a). Error estimates for CHUR compositions are from Bouvier et al (2008).

\subsection{G01/36}

The zircon grains from sample G01/36 are typically large (200-400 $\mu \mathrm{m}$ in length), prismatic or slightly oval in habit and display fine-scale oscillatory zoning occasionally cut by domains of recrystallisation (Electronic Annex EA8). Fifty-four $\mathrm{U}-\mathrm{Pb}$ spot analyses on 35 grains provided ${ }^{207} \mathrm{~Pb} /{ }^{206} \mathrm{~Pb}$ crystallisation ages ranging from 3914 to $3617 \mathrm{Ma}$ with a mean of $3880 \pm 8 \mathrm{Ma}(1 \sigma)$ from the 21 spots $>3850 \mathrm{Ma}$ (Fig. 6A). Th/U was typically moderate averaging 0.48 , with five analyses made on oscillatory zoned domains $<0.30$ (G01/36R-1.1, 8.3, 12.1, 17.1, 22.2). Two analyses on apparently recrystallised cores (G01/ 36R-16.3, 22.1) were indistinguishable in age and $\mathrm{Th} / \mathrm{U}$ from that clearly of igneous origin. $\delta^{18} \mathrm{O}$ from 31 analyses ranged from $4.1 \%$ to $6.2 \%$, with a weighted mean from 18 spots of $5.1 \pm 0.4 \%$ ( $95 \%$ c.l., MSWD $=2.3$, Fig. $6 \mathrm{~B})$. Four $\delta^{18} \mathrm{O}$ analyses of oscillatory zoned zircon with ages $<3800$ Ma (G01/36-2.1, 6.1, 7.1, 10.1) recorded compositions from $5.1 \%$ o to $6.1 \%$, consistent with spots dated at $>3800 \mathrm{Ma}$. $\varepsilon_{\mathrm{Hf}(\mathrm{T})}$ from 50 analyses ranged from 1.9 to -4.5 , with a weighted mean from 19 spots of $0.5 \pm 0.5$ $(95 \%$ c.l., MSWD $=0.6$, Fig. 6 C). A population of 39 analyses form a well defined $\varepsilon_{\mathrm{Hf}(\mathrm{T})}-{ }^{207} \mathrm{~Pb} /{ }^{206} \mathrm{~Pb}$ array starting from $0.5 \varepsilon_{\mathrm{Hf}(\mathrm{T})}$ units at $3882 \mathrm{Ma}$ to approximately $\varepsilon_{\mathrm{Hf}(\mathrm{T})}=-4.5$ at $\sim 3720 \mathrm{Ma}(\mathrm{G} 01 / 36-2.1,7.1)$, equivalent to a ${ }^{176} \mathrm{Lu} /{ }^{177} \mathrm{Hf}$ ratio of 0.007 (Fig. 6C). The other 15 analyses plot between this array and CHUR.

Given the large size of zircons in this sample, their extensive oscillatory domains and complex age structure, multiple analyses were performed on several grains to look for systematic trends in chemistry through their growth from core to rim. A compilation of 33 zircon CL images with analysis locations, crystallisation ages, \% discordance, Th/ $\mathrm{U}$ ratios, $\delta^{18} \mathrm{O}$ and $\varepsilon_{\mathrm{Hf}(\mathrm{T})}$ compositions are presented in Electronic Annex EA-8. Multiple analyses on 13 grains showed no clear correlations between $\delta^{18} \mathrm{O}$ or $\varepsilon_{\mathrm{Hf}(\mathrm{T})}$ and $\mathrm{Th} / \mathrm{U}$ ratio or growth domain (i.e. core, median or rim) as identified by CL imaging. Subtle relative isotopic shifts, both increasing and decreasing $\delta^{18} \mathrm{O}$ and $\varepsilon_{\mathrm{Hf}(\mathrm{T})}$ from grains core to rim were observed, however, such shifts were typically within analytical uncertainties.

\subsection{G01/113}

Sample G01/113 zircons are typically $100-200 \mu \mathrm{m}$ in length, prismatic to oval in habit and with fine-scale oscillatory zoning (Fig. 5A). Seventeen ${ }^{207} \mathrm{~Pb} /{ }^{206} \mathrm{~Pb}$ crystallisation ages on oscillatory zoned domains range from 3915 to $3674 \mathrm{Ma}$, with a mean of $3849 \pm 14 \mathrm{Ma}(1 \sigma$, Fig. 6D) and an average $\mathrm{Th} / \mathrm{U}$ ratio of 0.48 . Nine analyses of $\delta^{18} \mathrm{O}$ on oscillatory zoned zircon span a range of compositions from $4.2 \%$ (G01/113-2.1; Fig. 5A) to $6.3 \%$ (G01/113-13.1; Fig. 5A) with a mean of $4.9 \pm 0.7 \%$ oo $(1 \sigma$, Fig. $6 \mathrm{E}) . \varepsilon_{\mathrm{Hf}(\mathrm{T})}$ analyses $(n=13)$ ranged from 2.4 to -2.7 forming a well defined array from the weighted mean of $\varepsilon_{\mathrm{Hf}(\mathrm{T})} 1.3 \pm 0.7$ $(95 \%$ c.l., $\mathrm{MSWD}=0.4)$ at $3849 \mathrm{Ma}$, to $-2.7 \varepsilon_{\mathrm{Hf}(\mathrm{T})}=$ at $\sim 3.67 \mathrm{Ga}$ (oscillatory zoned G01/113-3.2; (Fig. 5A)), equivalent to a ${ }^{176} \mathrm{Lu} /{ }^{177} \mathrm{Hf}$ ratio of 0.009 (Fig. $6 \mathrm{~F}$ ). The analysis of recrystallised domain G01/113-12.1 (Fig. 5A) records a younger ${ }^{207} \mathrm{~Pb} /{ }^{206} \mathrm{~Pb}$ age of $3727 \pm 3 \mathrm{Ma}$ and initial $\varepsilon_{\mathrm{Hf}}$ of $-0.8 \pm 1.2$ units.

\section{3. $G 97 / 18$}

G97/18 zircons are $150-300 \mu \mathrm{m}$ in length, prismatic and with fine-scale oscillatory zoning with minor recrystallisation (Fig. $5 \mathrm{~B}) .{ }^{207} \mathrm{~Pb} /{ }^{206} \mathrm{~Pb}$ ages $(n=16)$ on oscillatory zoned domains ranged from 3850 to $3661 \mathrm{Ma}$, with an average Th/U ratio of 0.45 and a mean ${ }^{207} \mathrm{~Pb} /{ }^{206} \mathrm{~Pb}$ age from 13 analyses of $3816 \pm 7 \mathrm{Ma}(1 \sigma$, Fig. $6 \mathrm{G})$. Compositions from $12 \delta^{18} \mathrm{O}$ analyses ranged from $4.3 \%$ to $5.6 \%$ with a weighted mean of $5.0 \pm 0.2 \%$ o $(95 \%$ c.1., MSWD =0.6, (Fig. $6 \mathrm{H}))$. Initial $\varepsilon_{\mathrm{Hf}}(n=15)$ ranged from 0.7 to -2.7 with a weighted mean from 12 analyses of $-0.1 \pm 0.6(95 \%$ c.l., MSWD $=0.3)$, again defining a tight linear array starting from the chondritic reference line and with an implied ${ }^{176} \mathrm{Lu} /{ }^{177} \mathrm{Hf}$ ratio of 0.012 (Fig. 6I). Three analyses of oscillatory zoned spots with ages $<3.80$ Ga (e.g. G97/18-2.1, 4.1; (Fig. 5B)) recorded $\delta^{18} \mathrm{O}$ within the range of analyses made on oscillatory zoned zircon older than $3.80 \mathrm{Ga}$, but more negative $\varepsilon_{\mathrm{Hf}(\mathrm{T})}$ compositions from -1.2 to -1.6 epsilon units.

\subsection{8}

Zircons from sample 248228 are $100-300 \mu \mathrm{m}$ in length with prismatic to oval habit. Zircon with oscillatory zoning is cut in places by domains of recrystallisation. ${ }^{207} \mathrm{~Pb} /{ }^{206} \mathrm{~Pb}$ ages $(n=22)$ ranged from 3679 to $3710 \mathrm{Ma}$ with a weighted mean of $3693 \pm 3 \mathrm{Ma}(95 \%$ c.1., MSWD $=2.4$, Fig. $6 \mathrm{~J})$ and mean $\mathrm{Th} / \mathrm{U}$ of 0.36 . Thirteen $\delta^{18} \mathrm{O}$ analyses ranged from 
Table 1

Summary of $\mathrm{U}-\mathrm{Pb}, \delta^{18} \mathrm{O}$ and $\varepsilon_{\mathrm{Hf}(\mathrm{T})}$ results with mean and weighted mean ages and compositions for zircon and olivine samples.

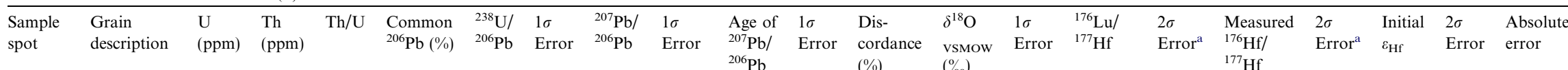

(Ma)

\begin{tabular}{|c|c|c|c|c|c|c|c|c|c|c|c|c|}
\hline \multicolumn{13}{|c|}{ G01/36 Meta-tonalite ( $64^{\circ} 18.21^{\prime} N 50^{\circ} 30.15^{\prime} \mathrm{W}-\mathrm{WGS}-84$ datum) } \\
\hline $1.1^{\mathrm{b}}$ & $\mathrm{pm}$ os & 159 & 75 & 0.48 & 0.018 & 1.215 & 0.031 & 0.3889 & 0.0011 & 3867 & 4 & 0 \\
\hline 1.2 & pe os & 78 & 26 & 0.35 & 0.021 & 1.250 & 0.033 & 0.3759 & 0.0036 & 3815 & 15 & 1 \\
\hline 2.1 & $\mathrm{p} \mathrm{m}$ os & 93 & 57 & 0.63 & 0.003 & 1.336 & 0.035 & 0.3528 & 0.0013 & 3719 & 6 & 3 \\
\hline 3.1 & ov e os & 73 & 38 & 0.54 & 0.000 & 1.252 & 0.033 & 0.3781 & 0.0015 & 3824 & 6 & 1 \\
\hline 4.1 & $\mathrm{pmos}$ & 137 & 94 & 0.71 & 0.104 & 1.320 & 0.034 & 0.3812 & 0.0017 & 3836 & 7 & 6 \\
\hline 4.2 & p mos & 51 & 21 & 0.43 & 0.020 & 1.256 & 0.033 & 0.3777 & 0.0021 & 3823 & 8 & 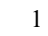 \\
\hline $5.1^{\mathrm{b}}$ & ov $\mathrm{m}$ os & 92 & 52 & 0.59 & 0.334 & 1.152 & 0.035 & 0.3906 & 0.0023 & 3873 & 9 & -4 \\
\hline 6.1 & p mos & 86 & 45 & 0.54 & 0.030 & 1.374 & 0.036 & 0.3497 & 0.0018 & 3706 & 8 & 5 \\
\hline 7.1 & p mos & 76 & 36 & 0.49 & 0.040 & 1.333 & 0.035 & 0.3538 & 0.0028 & 3723 & 12 & 3 \\
\hline $8.1^{\mathrm{b}}$ & $\mathrm{p} \mathrm{mos}$ & 74 & 40 & 0.56 & -0.007 & 1.243 & 0.032 & 0.3896 & 0.0049 & 3869 & 19 & 2 \\
\hline 9.1 & $\mathrm{p} \mathrm{mos}$ & 186 & 127 & 0.71 & 0.034 & 1.262 & 0.032 & 0.3792 & 0.0010 & 3828 & 4 & 2 \\
\hline 10.1 & ov e os & 131 & 50 & 0.39 & 0.018 & 1.336 & 0.034 & 0.3373 & 0.0011 & 3651 & 5 & 1 \\
\hline 11.1 & $\mathrm{pmos}$ & 55 & 22 & 0.42 & 0.000 & 1.248 & 0.034 & 0.3836 & 0.0024 & 3846 & 9 & 1 \\
\hline $12.1^{\mathrm{b}}$ & ov e os & 78 & 24 & 0.32 & 0.006 & 1.279 & 0.033 & 0.3891 & 0.0046 & 3868 & 18 & 4 \\
\hline 13.1 & $\mathrm{p} \mathrm{mos}$ & 75 & 29 & 0.39 & 0.034 & 1.241 & 0.032 & 0.3752 & 0.0021 & 3813 & 9 & 0 \\
\hline $\mathrm{R}-1.1$ & p e os & 146 & 17 & 0.12 & 0.127 & 1.299 & 0.013 & 0.3581 & 0.0027 & 3742 & 12 & 2 \\
\hline $\mathrm{R}-1.2$ & $\mathrm{pcos}$ & 65 & 21 & 0.34 & 0.094 & 1.260 & 0.017 & 0.3612 & 0.0030 & 3755 & 12 & 0 \\
\hline$R-2.1^{\mathrm{b}}$ & $\mathrm{pmos}$ & 146 & 62 & 0.44 & 0.256 & 1.239 & 0.012 & 0.3928 & 0.0025 & 3882 & 9 & 2 \\
\hline $\mathbf{R}-\mathbf{2} .2^{\mathrm{b}}$ & $\mathrm{pcos}$ & 132 & 55 & 0.43 & 0.100 & 1.223 & 0.013 & 0.4013 & 0.0020 & 3914 & 8 & 2 \\
\hline$R-2.3^{\mathrm{b}}$ & $\mathrm{pcos}$ & 183 & 72 & 0.40 & 0.073 & 1.229 & 0.010 & 0.3907 & 0.0024 & 3874 & 9 & 1 \\
\hline $\mathrm{R}-3.1$ & $\mathrm{p} \mathrm{mos}$ & 245 & 119 & 0.50 & 0.021 & 1.225 & 0.010 & 0.3835 & 0.0014 & 3846 & 6 & 0 \\
\hline R-3.3 & p mos & 148 & 52 & 0.36 & 0.052 & 1.264 & 0.013 & 0.3793 & 0.0026 & 3829 & 10 & 2 \\
\hline R-3.4 & $\mathrm{pmos}$ & 163 & 83 & 0.53 & 0.260 & 1.265 & 0.014 & 0.3815 & 0.0019 & 3838 & 7 & 2 \\
\hline$R-4.1^{b}$ & p mos & 263 & 148 & 0.58 & 0.471 & 1.345 & 0.010 & 0.3848 & 0.0015 & 3851 & 6 & 7 \\
\hline $\mathrm{R}-\mathbf{4} \cdot 2^{\mathrm{b}}$ & p mos & 199 & 87 & 0.45 & 0.090 & 1.223 & 0.010 & 0.3963 & 0.0016 & 3895 & 6 & 1 \\
\hline R-5.1 ${ }^{\mathrm{b}}$ & $\mathrm{p} \mathrm{mos}$ & 72 & 31 & 0.45 & 0.079 & 1.237 & 0.016 & 0.3955 & 0.0027 & 3892 & 10 & 2 \\
\hline R-5.2 $2^{\mathrm{b}}$ & p e os & 163 & 79 & 0.50 & 0.089 & 1.182 & 0.023 & 0.3938 & 0.0018 & 3886 & 7 & -2 \\
\hline R-6.1 & p m os & 130 & 55 & 0.44 & 0.083 & 1.312 & 0.013 & 0.3504 & 0.0021 & 3709 & 9 & 2 \\
\hline R-7.1 & $\mathrm{p} \mathrm{mos}$ & 206 & 115 & 0.58 & 0.133 & 1.146 & 0.020 & 0.3685 & 0.0019 & 3785 & 8 & -6 \\
\hline R-8.1 ${ }^{\mathrm{b}}$ & $\mathrm{p} \mathrm{mos}$ & 194 & 75 & 0.40 & 0.057 & 1.186 & 0.010 & 0.3999 & 0.0016 & 3909 & 6 & -1 \\
\hline R-8.2 ${ }^{\mathrm{b}}$ & $\mathrm{pcos}$ & 186 & 110 & 0.61 & 0.000 & 1.219 & 0.020 & 0.3950 & 0.0020 & 3890 & 8 & 1 \\
\hline R- 8.3 & pe os & 108 & 25 & 0.24 & 0.109 & 1.216 & 0.025 & 0.3664 & 0.0021 & 3777 & 9 & -2 \\
\hline R-9.1 ${ }^{\mathrm{b}}$ & $\mathrm{p} \mathrm{mos}$ & 264 & 130 & 0.51 & 0.046 & 1.171 & 0.009 & 0.3987 & 0.0013 & 3904 & 5 & -2 \\
\hline R-9..$^{\mathrm{b}}$ & $\mathrm{p} \mathrm{mos}$ & 180 & 61 & 0.35 & 0.073 & 1.235 & 0.010 & 0.3918 & 0.0017 & 3878 & 7 & 1 \\
\hline R-10.1 ${ }^{\mathrm{b}}$ & $\mathrm{pcos}$ & 118 & 57 & 0.50 & 0.106 & 1.214 & 0.013 & 0.3926 & 0.0020 & 3881 & 8 & 0 \\
\hline$R-10.2^{b}$ & $\mathrm{pcos}$ & 108 & 53 & 0.51 & 0.114 & 1.199 & 0.017 & 0.3920 & 0.0021 & 3879 & 8 & -1 \\
\hline$R-10.3^{b}$ & $\mathrm{p} \mathrm{mos}$ & 161 & 171 & 1.10 & 0.082 & 1.232 & 0.024 & 0.3914 & 0.0017 & 3876 & 7 & 1 \\
\hline$R-11.1^{b}$ & ov $\mathrm{m}$ os & 154 & 73 & 0.49 & 0.073 & 1.235 & 0.012 & 0.3855 & 0.0022 & 3854 & 9 & 1 \\
\hline R-12.1 & p m os & 254 & 31 & 0.1 & 0.0 & 1.262 & 0.010 & 0.3764 & 0.0014 & 3818 & 5 & 1 \\
\hline $\mathrm{R}-12.2$ & $\mathrm{pcos}$ & 322 & 122 & 0.39 & 0.466 & 1.353 & 0.025 & 0.3663 & 0.0016 & 3776 & 6 & 6 \\
\hline R-13.2 ${ }^{\mathrm{b}}$ & p mos & 294 & 199 & 0.70 & 0.089 & 1.189 & 0.008 & 0.3889 & 0.0014 & 3867 & 5 & -2 \\
\hline R-14.1 & p m os & 94 & 53 & 0.58 & 0.044 & 1.302 & 0.029 & 0.3300 & 0.0030 & 3617 & 14 & -2 \\
\hline R-15.1 & $\mathrm{p} \mathrm{mos}$ & 228 & 170 & 0.77 & 0.026 & 1.289 & 0.011 & 0.3756 & 0.0015 & 3814 & 6 & 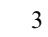 \\
\hline R-16.1 & $\mathrm{pe}$ os & 220 & 151 & 0.71 & 0.085 & 1.224 & 0.011 & 0.3822 & 0.0015 & 3841 & 6 & 0 \\
\hline
\end{tabular}

\begin{tabular}{|c|c|c|c|c|c|c|c|c|}
\hline 5.6 & 1.0 & 0.000348 & 24 & 0.280277 & 21 & -0.7 & 0.7 & 2.1 \\
\hline 5.0 & 0.7 & 0.000342 & 15 & 0.280271 & 21 & -2.1 & 0.8 & 2.1 \\
\hline 6.1 & 1.0 & 0.000443 & 10 & 0.280275 & 23 & -4.5 & 0.8 & 2.1 \\
\hline 6.2 & 1.0 & 0.000408 & 23 & 0.280299 & 28 & -1.0 & 1.0 & 2.2 \\
\hline 4.5 & 0.7 & 0.000412 & 13 & 0.280284 & 28 & -1.3 & 1.0 & 2.2 \\
\hline 5.0 & 0.7 & 0.000444 & 12 & 0.280309 & 25 & -0.8 & 0.9 & 2.1 \\
\hline 5.0 & 0.7 & 0.000433 & 22 & 0.280274 & 21 & -0.8 & 0.8 & 2.1 \\
\hline 5.1 & 0.7 & 0.000587 & 17 & 0.280317 & 23 & -3.7 & 0.8 & 2.1 \\
\hline 5.2 & 0.7 & 0.000567 & 11 & 0.280286 & 27 & -4.3 & 1.0 & 2.2 \\
\hline 4.1 & 0.7 & 0.000518 & 35 & 0.280300 & 23 & -0.2 & 0.8 & 2.1 \\
\hline 4.8 & 0.7 & 0.000814 & 89 & 0.280341 & 25 & -0.5 & 0.9 & 2.1 \\
\hline 5.8 & 0.7 & 0.000435 & 10 & 0.280387 & 24 & -2.1 & 0.9 & 2.1 \\
\hline 5.4 & 0.7 & 0.000270 & 2 & 0.280299 & 20 & -0.2 & 0.7 & 2.1 \\
\hline \multirow[t]{3}{*}{4.5} & 0.7 & 0.000215 & 2 & 0.280305 & 21 & 0.7 & 0.7 & 2.1 \\
\hline & & 0.000191 & 3 & 0.280341 & 41 & -1.0 & 1.5 & 2.4 \\
\hline & & 0.000327 & 8 & 0.280324 & 39 & -1.6 & 1.4 & 2.4 \\
\hline 5.4 & 0.4 & 0.000460 & 14 & 0.280346 & 45 & 1.9 & 1.6 & 2.5 \\
\hline 5.8 & 0.5 & & & & & & & \\
\hline 6.0 & 0.5 & 0.000605 & 16 & 0.280365 & 42 & 1.9 & 1.5 & 2.5 \\
\hline 5.4 & 0.5 & 0.000372 & 4 & 0.280286 & 32 & -0.9 & 1.1 & 2.2 \\
\hline 5.4 & 0.5 & 0.000746 & 22 & 0.280304 & 38 & -1.7 & 1.3 & 2.4 \\
\hline 5.4 & 0.5 & 0.000386 & 5 & 0.280261 & 32 & -2.0 & 1.2 & 2.3 \\
\hline 4.1 & 0.5 & 0.000496 & 7 & 0.280318 & 49 & 0.0 & 1.7 & 2.6 \\
\hline 4.3 & 0.5 & 0.000477 & 4 & 0.280303 & 47 & 0.6 & 1.7 & 2.6 \\
\hline 4.2 & 0.5 & 0.000547 & 11 & 0.280305 & 36 & 0.4 & 1.3 & 2.3 \\
\hline \multirow[t]{3}{*}{5.7} & 0.5 & 0.000240 & 10 & 0.280275 & 30 & 0.0 & 1.1 & 2.2 \\
\hline & & 0.000469 & 10 & 0.280388 & 48 & -0.8 & 1.7 & 2.6 \\
\hline & & 0.000716 & 18 & 0.280318 & 44 & -2.1 & 1.6 & 2.5 \\
\hline 4.4 & 0.5 & 0.000488 & 12 & 0.280309 & 41 & 1.1 & 1.4 & 2.4 \\
\hline \multirow[t]{2}{*}{4.3} & 0.5 & 0.000827 & 12 & 0.280312 & 53 & -0.1 & 1.9 & 2.7 \\
\hline & & 0.000280 & 7 & 0.280299 & 45 & -1.8 & 1.6 & 2.5 \\
\hline 4.6 & 0.5 & 0.000553 & 7 & 0.280334 & 45 & 1.7 & 1.6 & 2.5 \\
\hline 5.8 & 0.5 & & & & & & & \\
\hline 6.2 & 0.5 & 0.000703 & 15 & 0.280347 & 46 & 1.2 & 1.6 & 2.5 \\
\hline 5.2 & 0.5 & 0.000697 & 11 & 0.280343 & 57 & 1.0 & 2.0 & 2.8 \\
\hline \multirow[t]{8}{*}{6.2} & 0.5 & 0.000367 & 7 & 0.280268 & 52 & -0.8 & 1.9 & 2.7 \\
\hline & & 0.000545 & 21 & 0.280351 & 53 & 1.1 & 1.9 & 2.7 \\
\hline & & 0.000325 & 4 & 0.280317 & 38 & -0.3 & 1.4 & 2.4 \\
\hline & & 0.000509 & 9 & 0.280373 & 50 & 0.2 & 1.8 & 2.6 \\
\hline & & 0.000271 & 8 & 0.280321 & 47 & 1.1 & 1.7 & 2.6 \\
\hline & & 0.000433 & 35 & 0.280456 & 49 & -0.4 & 1.8 & 2.6 \\
\hline & & 0.000228 & 16 & 0.280335 & 32 & 0.5 & 1.1 & 2.3 \\
\hline & & 0.000600 & 26 & 0.280333 & 35 & 0.1 & 1.3 & 2.3 \\
\hline
\end{tabular}




\begin{tabular}{|c|c|c|c|c|c|c|c|c|c|c|c|c|c|c|c|c|c|c|c|c|c|}
\hline $\begin{array}{l}\text { Sample } \\
\text { spot }\end{array}$ & $\begin{array}{l}\text { Grain } \\
\text { description }\end{array}$ & $\begin{array}{l}\mathrm{U} \\
(\mathrm{ppm})\end{array}$ & $\begin{array}{l}\text { Th } \\
(\mathrm{ppm})\end{array}$ & $\mathrm{Th} / \mathrm{U}$ & $\begin{array}{l}\text { Common } \\
{ }^{206} \mathrm{~Pb}(\%)\end{array}$ & ${ }^{238} \mathrm{U} /$ & $\begin{array}{l}1 \sigma \\
\text { Error }\end{array}$ & $\begin{array}{l}{ }^{207} \mathrm{~Pb} / \\
{ }^{206} \mathrm{~Pb}\end{array}$ & $\begin{array}{l}1 \sigma \\
\text { Error }\end{array}$ & $\begin{array}{l}\text { Age of } \\
{ }^{207} \mathrm{~Pb} / \\
{ }^{206} \mathrm{~Pb} \\
(\mathrm{Ma})\end{array}$ & $\begin{array}{l}1 \sigma \\
\text { Error }\end{array}$ & $\begin{array}{l}\text { Dis- } \\
\text { cordance } \\
(\%)\end{array}$ & $\begin{array}{l}\delta^{18} \mathrm{O} \\
\text { vSMOw } \\
(\%)\end{array}$ & $\begin{array}{l}1 \sigma \\
\text { Error }\end{array}$ & ${ }^{176} \mathrm{Lu} /$ & $\begin{array}{l}2 \sigma \\
\text { Error }^{\mathrm{a}}\end{array}$ & $\begin{array}{l}\text { Measured } \\
{ }^{176} \mathrm{Hf} / \\
{ }^{177} \mathrm{Hf}\end{array}$ & $\begin{array}{l}2 \sigma \\
\text { Error }^{\mathrm{a}}\end{array}$ & $\begin{array}{l}\text { Initial } \\
\varepsilon_{\mathrm{Hf}}\end{array}$ & $\begin{array}{l}2 \sigma \\
\text { Error }\end{array}$ & $\begin{array}{l}\text { Absolute } \\
\text { error }\end{array}$ \\
\hline R-16.2 & $\mathrm{p} \mathrm{m}$ os & 85 & 48 & 0.59 & 0.496 & 1.259 & 0.017 & 0.3792 & 0.0026 & 3829 & 11 & 2 & & & 0.000805 & 44 & 0.280337 & 46 & -0.6 & 1.6 & 2.5 \\
\hline R-16.3 & $\mathrm{p} \mathrm{c} \mathrm{rx}$ & 41 & 19 & 0.48 & 0.208 & 1.299 & 0.031 & 0.3659 & 0.0035 & 3774 & 14 & 3 & & & 0.000417 & 32 & 0.280342 & 39 & -0.7 & 1.4 & 2.4 \\
\hline R-17.1 & $\mathrm{p} \mathrm{mos}$ & 250 & 62 & 0.26 & 0.302 & 1.313 & 0.011 & 0.3638 & 0.0018 & 3766 & 7 & 3 & & & & & & & & & \\
\hline R-18.1 $1^{b}$ & $\mathrm{pcos}$ & 222 & 112 & 0.52 & 0.224 & 1.205 & 0.009 & 0.3906 & 0.0016 & 3873 & 6 & -1 & & & 0.000738 & 32 & 0.280367 & 46 & 1.7 & 1.6 & 2.5 \\
\hline R-18.2 & $\mathrm{p} \mathrm{m}$ os & 49 & 20 & 0.41 & 0.208 & 1.321 & 0.020 & 0.3417 & 0.0030 & 3670 & 13 & 1 & & & 0.000129 & 6 & 0.280392 & 33 & -0.7 & 1.2 & 2.3 \\
\hline R-19.1 & $\mathrm{pmos}$ & 89 & 44 & 0.51 & 0.194 & 1.346 & 0.015 & 0.3310 & 0.0039 & 3622 & 18 & 1 & & & 0.000276 & 10 & 0.280412 & 43 & -1.5 & 1.5 & 2.5 \\
\hline R-20.1 & $\mathrm{p} \mathrm{m}$ os & 103 & 44 & 0.44 & 0.156 & 1.296 & 0.014 & 0.3363 & 0.0023 & 3646 & 10 & -1 & & & 0.000252 & 2 & 0.280349 & 38 & -3.1 & 1.4 & 2.4 \\
\hline R-21.1 & $\mathrm{p} \mathrm{mos}$ & 136 & 50 & 0.38 & 0.043 & 1.218 & 0.013 & 0.3645 & 0.0029 & 3769 & 12 & -2 & & & 0.000264 & 2 & 0.280290 & 36 & -2.3 & 1.3 & 2.3 \\
\hline $\mathrm{R}-22.1$ & ov c rx & 230 & 154 & 0.69 & 0.074 & 1.210 & 0.023 & 0.3827 & 0.0014 & 3842 & 6 & -1 & & & 0.000544 & 35 & 0.280289 & 36 & -1.3 & 1.3 & 2.3 \\
\hline $\mathrm{R}-22.2$ & ov $\mathrm{m}$ os & 189 & 38 & 0.21 & 0.025 & 1.207 & 0.023 & 0.3834 & 0.0016 & 3845 & 6 & -1 & & & 0.000431 & 13 & 0.280253 & 37 & -2.2 & 1.3 & 2.3 \\
\hline \multicolumn{10}{|c|}{$\begin{array}{l}\text { Weighted mean }{ }^{\mathrm{c}} \pm 95 \% \text { confidence limits or mean }{ }^{\mathrm{d}} \pm 1 \sigma \\
\end{array}$} & $\begin{array}{l}3880^{\mathrm{d}} \\
21 \text { of } 54\end{array}$ & 8 & & $\begin{array}{l}\mathbf{5 . 1} \mathbf{1}^{\mathrm{c}} \\
18 \text { of } 31 \\
\mathbf{2 . 3}\end{array}$ & 0.4 & & & & & $\begin{array}{l}\mathbf{0 . 5} \mathbf{5}^{\mathrm{c}} \\
19 \text { of } 50 \\
\mathbf{0 . 6}\end{array}$ & & 0.5 \\
\hline \multicolumn{22}{|c|}{ G011113 Meta-tonalite $\left(63^{\circ} 55.68^{\prime} \mathrm{N} 51^{\circ} 43.60^{\prime} \mathrm{W}\right)$} \\
\hline $1.1^{\mathrm{b}}$ & $\mathrm{p} \mathrm{mos}$ & 278 & 164 & 0.61 & 0.030 & 1.167 & 0.012 & 0.3866 & 0.0009 & 3858 & 3 & -3 & 4.9 & 0.4 & 0.000589 & 19 & 0.280357 & 35 & 1.3 & 1.3 & 2.3 \\
\hline $2.1^{\mathrm{b}}$ & $\mathrm{pmos}$ & 256 & 130 & 0.52 & 0.205 & 1.189 & 0.012 & 0.3860 & 0.0012 & 3856 & 5 & -2 & 4.2 & 0.4 & 0.000502 & 19 & 0.280381 & 37 & 2.4 & 1.3 & 2.3 \\
\hline $3.1^{\mathrm{b}}$ & $\mathrm{p} \mathrm{m}$ os & 217 & 125 & 0.60 & 0.183 & 1.208 & 0.013 & 0.3795 & 0.0012 & 3830 & 5 & -1 & & & 0.000455 & 21 & 0.280372 & 41 & 1.6 & 1.5 & 2.4 \\
\hline 3.2 & $\mathrm{p} \mathrm{m}$ os & 146 & 30 & 0.21 & 0.011 & 1.426 & 0.016 & 0.3426 & 0.0036 & 3674 & 16 & 7 & & & 0.000427 & 6 & 0.280353 & 41 & -2.7 & 1.5 & 2.4 \\
\hline $4.1^{\mathrm{b}}$ & $\mathrm{p} \mathrm{mos}$ & 153 & 58 & 0.39 & 0.028 & 1.239 & 0.013 & 0.3881 & 0.0009 & 3864 & 3 & 1 & 4.4 & 0.4 & 0.000631 & 9 & 0.280354 & 34 & 1.3 & 1.2 & 2.3 \\
\hline $5.1^{\mathrm{b}}$ & $\mathrm{p} \mathrm{m}$ os & 257 & 129 & 0.52 & 0.034 & 1.191 & 0.013 & 0.3880 & 0.0017 & 3863 & 7 & -2 & 5.6 & 0.4 & 0.000593 & 5 & 0.280378 & 36 & 2.2 & 1.3 & 2.3 \\
\hline $6.1^{\mathrm{b}}$ & ov m os & 239 & 184 & 0.79 & 0.017 & 1.310 & 0.014 & 0.3777 & 0.0012 & 3823 & 5 & 5 & 4.7 & 0.4 & & & & & & & \\
\hline 7.1 & $\mathrm{p} \mathrm{mos}$ & 236 & 147 & 0.64 & 0.031 & 1.216 & 0.013 & 0.3719 & 0.0012 & 3799 & 5 & -2 & & & & & & & & & \\
\hline $7.2^{\mathrm{b}}$ & $\mathrm{p} \mathrm{mos}$ & 250 & 53 & 0.22 & 0.080 & 1.273 & 0.020 & 0.3859 & 0.0016 & 3855 & 6 & 3 & 4.3 & 0.4 & 0.000643 & 28 & 0.280356 & 35 & 1.1 & 1.3 & 2.3 \\
\hline $8.1^{\mathrm{b}}$ & $\mathrm{p} \mathrm{mos}$ & 372 & 202 & 0.56 & 0.005 & 1.223 & 0.013 & 0.3740 & 0.0008 & 3808 & 3 & -1 & 5.2 & 0.4 & 0.000536 & 5 & 0.280366 & 30 & 0.6 & 1.1 & 2.2 \\
\hline $10.1^{\mathrm{b}}$ & $\mathrm{pmos}$ & 147 & 55 & 0.39 & 0.081 & 1.247 & 0.014 & 0.3819 & 0.0008 & 3839 & 3 & 1 & & & 0.000474 & 11 & 0.280359 & 36 & 1.3 & 1.3 & 2.3 \\
\hline $11.1^{\mathrm{b}}$ & pe os & 188 & 85 & 0.46 & -0.009 & 1.287 & 0.035 & 0.3781 & 0.0047 & 3824 & 19 & 3 & & & 0.000674 & 11 & 0.280338 & 33 & -0.4 & 1.2 & 2.3 \\
\hline 12.1 & $\mathrm{p} \mathrm{m} \mathrm{rx}$ & 226 & 42 & 0.19 & 0.186 & 1.354 & 0.014 & 0.3546 & 0.0007 & 3727 & 3 & 5 & & & 0.000441 & 12 & 0.280373 & 34 & -0.8 & 1.2 & 2.3 \\
\hline $13.1^{\mathrm{b}}$ & $\mathrm{p} \mathrm{mos}$ & 322 & 128 & 0.41 & 0.004 & 1.218 & 0.012 & 0.3863 & 0.0012 & 3856 & 5 & 0 & 6.3 & 0.4 & 0.000471 & 21 & 0.280362 & 37 & 1.8 & 1.3 & 2.3 \\
\hline $15.1^{\mathrm{b}}$ & ov $\mathrm{m}$ os & 166 & 89 & 0.56 & 0.006 & 1.209 & 0.013 & 0.3919 & 0.0008 & 3878 & 3 & 0 & 4.5 & 0.4 & 0.000545 & 2 & 0.280353 & 46 & 1.8 & 1.6 & 2.5 \\
\hline $16.1^{\mathrm{b}}$ & $\mathrm{p} \mathrm{mos}$ & 234 & 127 & 0.56 & 0.022 & 1.291 & 0.014 & 0.4015 & 0.0087 & 3915 & 33 & 6 & & & & & & & & & \\
\hline $16.2^{\mathrm{b}}$ & $\mathrm{pmos}$ & 210 & 99 & 0.49 & -0.002 & 1.195 & 0.052 & 0.3861 & 0.0074 & 3856 & 29 & -2 & & & & & & & & & \\
\hline \multicolumn{10}{|c|}{$\begin{array}{l}\text { Weighted } \text { mean }^{\mathrm{c}} \pm 95 \% \text { confidence limits or } \text { mean }^{\mathrm{d}} \pm 1 \sigma \\
n \\
\text { MSWD }\end{array}$} & $\begin{array}{l}3849^{\mathrm{d}} \\
14 \text { of } 17\end{array}$ & 14 & & $\begin{array}{l}4.9^{\mathrm{d}} \\
9 \text { of } 9\end{array}$ & 0.7 & & & & & $\begin{array}{l}1.3^{\mathrm{c}} \\
11 \text { of } 13 \\
\mathbf{0 . 4}\end{array}$ & & 0.7 \\
\hline \multicolumn{22}{|c|}{ G97/18 Meta-tonalite $\left(65^{\circ} 00.63^{\prime} \mathrm{N} 50^{\circ} 15.04^{\prime} \mathrm{W}\right)$} \\
\hline $1.1^{\mathrm{b}}$ & $\mathrm{pm}$ os & 216 & 139 & 0.67 & 0.006 & 1.206 & 0.020 & 0.3762 & 0.0009 & 3817 & 4 & -2 & 5.5 & 0.6 & 0.000503 & 11 & 0.280358 & 23 & 0.6 & 0.8 & 2.1 \\
\hline 2.1 & $\mathrm{p} \mathrm{m}$ os & 87 & 33 & 0.39 & 0.017 & 1.253 & 0.021 & 0.3655 & 0.0013 & 3773 & 5 & 0 & 4.7 & 0.6 & 0.000320 & 13 & 0.280323 & 20 & -1.2 & 0.7 & 2.1 \\
\hline $3.1^{\mathrm{b}}$ & $\mathrm{p} \mathrm{m}$ os & 93 & 36 & 0.40 & 0.005 & 1.254 & 0.021 & 0.3720 & 0.0012 & 3800 & 5 & 1 & & & 0.000403 & 7 & 0.280320 & 23 & -0.9 & 0.8 & 2.1 \\
\hline 4.1 & $\mathrm{p} \mathrm{mos}$ & 158 & 46 & 0.30 & -0.011 & 1.344 & 0.022 & 0.3517 & 0.0015 & 3714 & 6 & 4 & 5.6 & 0.6 & 0.000396 & 22 & 0.280356 & 23 & -1.6 & 0.8 & 2.1 \\
\hline $5.1^{\mathrm{b}}$ & $\mathrm{p} \mathrm{m}$ os & 147 & 91 & 0.64 & 0.056 & 1.271 & 0.023 & 0.3758 & 0.0012 & 3815 & 5 & 2 & 4.3 & 0.6 & 0.000463 & 10 & 0.280327 & 20 & -0.4 & 0.7 & 2.1 \\
\hline $5.2^{\mathrm{b}}$ & $\mathrm{pcos}$ & 121 & 75 & 0.64 & 0.037 & 1.358 & 0.022 & 0.3758 & 0.0011 & 3815 & 4 & 7 & & & & & & & & & \\
\hline $6.1^{\mathrm{b}}$ & $\mathrm{pmos}$ & 135 & 61 & 0.47 & -0.006 & 1.327 & 0.022 & 0.3735 & 0.0013 & 3806 & 5 & 5 & 5.6 & 0.6 & 0.000423 & 29 & 0.280353 & 28 & 0.4 & 1.0 & 2.2 \\
\hline $7.1^{\mathrm{b}}$ & $\mathrm{p} \mathrm{m}$ os & 231 & 131 & 0.59 & 0.010 & 1.246 & 0.015 & 0.3790 & 0.0009 & 3828 & 3 & 1 & & & 0.000450 & 15 & 0.280317 & 29 & -0.4 & 1.0 & 2.2 \\
\hline $8.1^{\mathrm{b}}$ & $\mathrm{p} \mathrm{m}$ os & 63 & 22 & 0.36 & 0.099 & 1.240 & 0.014 & 0.3787 & 0.0016 & 3826 & 6 & 0 & & & 0.000520 & 9 & 0.280355 & 29 & 0.7 & 1.0 & 2.2 \\
\hline $9.1^{\mathrm{b}}$ & $\mathrm{p} \mathrm{m}$ os & 179 & 68 & 0.39 & 0.025 & 1.241 & 0.011 & 0.3786 & 0.0011 & 3826 & 4 & 0 & 4.7 & 0.3 & 0.000369 & 16 & 0.280317 & 31 & -0.3 & 1.1 & 2.2 \\
\hline 10.1 & $\mathrm{p} \mathrm{m}$ os & 97 & 26 & 0.28 & 0.055 & 1.341 & 0.014 & 0.3397 & 0.0013 & 3661 & 6 & 2 & 4.7 & 0.3 & 0.000517 & 8 & 0.280368 & 31 & -2.7 & 1.1 & 2.2 \\
\hline $11.1^{\mathrm{b}}$ & $\mathrm{p} \mathrm{m}$ os & 117 & 42 & 0.38 & 0.028 & 1.251 & 0.012 & 0.3758 & 0.0012 & 3815 & 5 & 1 & 5.0 & 0.3 & 0.000460 & 4 & 0.280344 & 31 & 0.2 & 1.1 & 2.2 \\
\hline
\end{tabular}




\begin{tabular}{|c|c|c|c|c|c|c|c|c|c|c|}
\hline $12.1^{\mathrm{b}}$ & $\mathrm{p} \mathrm{m}$ os & 179 & 71 & 0.41 & 0.012 & 1.270 & 0.013 & 0.3763 & 0.0010 & 3817 \\
\hline $13.1^{\mathrm{b}}$ & $\mathrm{p} \mathrm{m}$ os & 123 & 36 & 0.30 & -0.002 & 1.218 & 0.036 & 0.3847 & 0.0021 & 3850 \\
\hline $14.1^{\mathrm{b}}$ & $\mathrm{p} \mathrm{m}$ os & 135 & 59 & 0.45 & -0.002 & 1.232 & 0.011 & 0.3759 & 0.0011 & 3816 \\
\hline $15.1^{\mathrm{b}}$ & $\mathrm{p} \mathrm{m}$ os & 140 & 74 & 0.55 & 0.006 & 1.232 & 0.015 & 0.3723 & 0.0009 & 3801 \\
\hline \multicolumn{10}{|c|}{$\begin{array}{l}\text { Weighted } \text { mean }^{\mathrm{c}} \pm 95 \% \text { confidence limits or mean }{ }^{\mathrm{d}} \pm 1 \sigma \\
n\end{array}$} & $\begin{array}{l}\mathbf{3 8 1 6}^{\mathrm{d}} \\
13 \text { of } 16\end{array}$ \\
\hline \multicolumn{11}{|c|}{ MSWD } \\
\hline \multicolumn{11}{|c|}{248228 Meta-tonalite $\left(65^{\circ} 07.00^{\prime} N 49^{\circ} 57.42^{\prime} \mathrm{W}\right)$} \\
\hline $1.1^{\mathrm{b}}$ & $\mathrm{p} \mathrm{m}$ os & 117 & 44 & 0.39 & 0.014 & 1.266 & 0.015 & 0.3458 & 0.0011 & 3689 \\
\hline $2.1^{\mathrm{b}}$ & $\mathrm{p} \mathrm{m}$ os & 149 & 68 & 0.47 & 0.006 & 1.268 & 0.015 & 0.3507 & 0.0010 & 3710 \\
\hline $3.1^{\mathrm{b}}$ & $\mathrm{pcos}$ & 235 & 69 & 0.30 & 0.008 & 1.266 & 0.014 & 0.3468 & 0.0008 & 3693 \\
\hline $4.1^{\mathrm{b}}$ & $\mathrm{pcos}$ & 101 & 16 & 0.16 & 0.046 & 1.279 & 0.019 & 0.3442 & 0.0012 & 3681 \\
\hline $5.1^{\mathrm{b}}$ & ov c rx & 110 & 57 & 0.54 & 0.023 & 1.308 & 0.016 & 0.3488 & 0.0012 & 3702 \\
\hline $6.1^{\mathrm{b}}$ & $\mathrm{p} \mathrm{mos}$ & 122 & 50 & 0.42 & 0.063 & 1.277 & 0.015 & 0.3458 & 0.0017 & 3689 \\
\hline $7.1^{\mathrm{b}}$ & $\mathrm{pcos}$ & 135 & 55 & 0.42 & 0.025 & 1.271 & 0.020 & 0.3451 & 0.0011 & 3686 \\
\hline $8.1^{\mathrm{b}}$ & $\mathrm{p} \mathrm{m}$ os & 171 & 42 & 0.26 & 0.022 & 1.257 & 0.014 & 0.3465 & 0.0009 & 3692 \\
\hline $9.1^{\mathrm{b}}$ & $\mathrm{p} \mathrm{mos}$ & 167 & 89 & 0.55 & 0.010 & 1.278 & 0.015 & 0.3467 & 0.0009 & 3692 \\
\hline $10.1^{\mathrm{b}}$ & $\mathrm{p} \mathrm{mos}$ & 137 & 75 & 0.57 & 0.032 & 1.309 & 0.015 & 0.3436 & 0.0010 & 3679 \\
\hline $11.1^{\mathrm{b}}$ & $\mathrm{p} \mathrm{m}$ os & 183 & 92 & 0.52 & 0.012 & 1.262 & 0.014 & 0.3454 & 0.0009 & 3687 \\
\hline $12.1^{\mathrm{b}}$ & $\mathrm{p} \mathrm{mos}$ & 169 & 30 & 0.19 & 0.029 & 1.326 & 0.015 & 0.3476 & 0.0012 & 3697 \\
\hline $13.1^{\mathrm{b}}$ & $\mathrm{pcos}$ & 101 & 15 & 0.15 & 0.037 & 1.289 & 0.016 & 0.3464 & 0.0012 & 3691 \\
\hline $14.1^{\mathrm{b}}$ & $\mathrm{p} \mathrm{mos}$ & 205 & 35 & 0.18 & 0.008 & 1.270 & 0.014 & 0.3469 & 0.0011 & 3693 \\
\hline $15.1^{\mathrm{b}}$ & $\mathrm{p} \mathrm{mos}$ & 257 & 109 & 0.44 & 0.033 & 1.324 & 0.015 & 0.3480 & 0.0008 & 3698 \\
\hline $16.1^{\mathrm{b}}$ & $\mathrm{p} \mathrm{mos}$ & 200 & 77 & 0.40 & 0.021 & 1.300 & 0.015 & 0.3456 & 0.0009 & 3688 \\
\hline $17.1^{\mathrm{b}}$ & $\mathrm{p} \mathrm{mos}$ & 106 & 37 & 0.36 & 0.024 & 1.277 & 0.016 & 0.3466 & 0.0012 & 3692 \\
\hline $18.1^{\mathrm{b}}$ & $\mathrm{p} \mathrm{mos}$ & 216 & 33 & 0.16 & -0.003 & 1.262 & 0.014 & 0.3480 & 0.0009 & 3698 \\
\hline $18.2^{\mathrm{b}}$ & $\mathrm{p} \mathrm{m}$ os & 163 & 53 & 0.33 & 0.017 & 1.285 & 0.015 & 0.3486 & 0.0009 & 3701 \\
\hline $21.1^{\mathrm{b}}$ & $\mathrm{p} \mathrm{mos}$ & 306 & 142 & 0.48 & 0.000 & 1.273 & 0.014 & 0.3465 & 0.0019 & 3692 \\
\hline $22.1^{\mathrm{b}}$ & $\mathrm{p} \mathrm{mos}$ & 150 & 60 & 0.41 & 0.014 & 1.270 & 0.015 & 0.3481 & 0.0010 & 3699 \\
\hline $23.1^{\mathrm{b}}$ & $\mathrm{p} \mathrm{mos}$ & 46 & 14 & 0.32 & 0.000 & 1.265 & 0.018 & 0.3479 & 0.0018 & 3698 \\
\hline \multicolumn{10}{|c|}{ Weighted mean ${ }^{\mathrm{c}} \pm 95 \%$ confidence limits or mean ${ }^{\mathrm{d}} \pm 1 \sigma$} & $3693^{\mathrm{c}}$ \\
\hline$n$ & & & & & & & & & & 22 of 22 \\
\hline MSWI & & & & & & & & & & 2.4 \\
\hline \multicolumn{11}{|c|}{248202 Felsic meta-volcanic $\left(65^{\circ} 05.83^{\prime} N 50^{\circ} 00.00^{\prime} W\right)$} \\
\hline $1.1^{\mathrm{b}}$ & $\mathrm{p} \mathrm{m}$ os & 111 & 53 & 0.49 & -0.030 & 1.331 & 0.007 & 0.3739 & 0.0009 & 3807 \\
\hline $2.1^{\mathrm{b}}$ & p e os & 320 & 233 & 0.75 & -0.019 & 1.283 & 0.008 & 0.3732 & 0.0006 & 3805 \\
\hline $3.1^{\mathrm{b}}$ & $\mathrm{p} \mathrm{m}$ os & 193 & 157 & 0.84 & -0.003 & 1.241 & 0.006 & 0.3732 & 0.0007 & 3805 \\
\hline $4.1^{\mathrm{b}}$ & eq $\mathrm{m}$ os & 249 & 149 & 0.62 & -0.007 & 1.230 & 0.005 & 0.3733 & 0.0006 & 3805 \\
\hline $5.1^{\mathrm{b}}$ & ov $\mathrm{m}$ os & 93 & 54 & 0.60 & -0.013 & 1.266 & 0.007 & 0.3740 & 0.0009 & 3808 \\
\hline $6.1^{\mathrm{b}}$ & $\mathrm{p} \mathrm{cos}$ & 140 & 75 & 0.55 & 0.003 & 1.273 & 0.007 & 0.3735 & 0.0012 & 3806 \\
\hline $7.1^{\mathrm{b}}$ & $\mathrm{p} \mathrm{mos}$ & 88 & 34 & 0.40 & -0.008 & 1.286 & 0.009 & 0.3719 & 0.0011 & 3799 \\
\hline $8.1^{\mathrm{b}}$ & p c rx & 132 & 61 & 0.48 & 0.039 & 1.301 & 0.019 & 0.3747 & 0.0010 & 3811 \\
\hline $9.1^{\mathrm{b}}$ & $\mathrm{p} \mathrm{mos}$ & 114 & 59 & 0.54 & 0.001 & 1.256 & 0.007 & 0.3721 & 0.0010 & 3800 \\
\hline 10.1 & $\mathrm{p} \mathrm{mos}$ & 199 & 78 & 0.41 & 0.080 & 1.230 & 0.005 & 0.3694 & 0.0007 & 3789 \\
\hline $11.1^{\mathrm{b}}$ & eq cos & 442 & 422 & 0.99 & 0.010 & 1.230 & 0.004 & 0.3742 & 0.0005 & 3809 \\
\hline 12.1 & an c rx & 91 & 49 & 0.56 & 0.068 & 1.290 & 0.008 & 0.3644 & 0.0011 & 3768 \\
\hline $13.1^{\mathrm{b}}$ & eq $\mathrm{m}$ os & 122 & 51 & 0.43 & 0.000 & 1.248 & 0.007 & 0.3724 & 0.0010 & 3801 \\
\hline $14.1^{\mathrm{b}}$ & $\mathrm{pcos}$ & 247 & 127 & 0.53 & 0.019 & 1.259 & 0.006 & 0.3727 & 0.0007 & 3803 \\
\hline $15.1^{\mathrm{b}}$ & eq c os & 130 & 61 & 0.48 & 0.003 & 1.251 & 0.029 & 0.3732 & 0.0009 & 3804 \\
\hline $16.1^{\mathrm{b}}$ & $\mathrm{pcos}$ & 137 & 51 & 0.39 & 0.003 & 1.257 & 0.018 & 0.3727 & 0.0009 & 3802 \\
\hline $17.1^{\mathrm{b}}$ & $\mathrm{pcos}$ & 220 & 97 & 0.46 & 0.013 & 1.222 & 0.006 & 0.3739 & 0.0008 & 3807 \\
\hline $18.1^{\mathrm{b}}$ & & 9 & 71 & 0.41 & 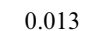 & 1.172 & 12 & & 0.0013 & 06 \\
\hline
\end{tabular}

\begin{tabular}{|c|c|c|c|c|c|c|c|c|}
\hline 5.0 & 0.3 & 0.000467 & 17 & 0.280319 & 29 & -0.7 & 1.0 & 2.2 \\
\hline 5.0 & 0.3 & 0.000462 & 9 & 0.280328 & 31 & 0.5 & 1.1 & 2.2 \\
\hline 5.2 & 0.3 & 0.000489 & 11 & 0.280314 & 30 & -0.9 & 1.1 & 2.2 \\
\hline 4.8 & 0.3 & 0.000488 & 14 & 0.280345 & 31 & -0.2 & 1.1 & 2.2 \\
\hline $5.0^{\mathrm{c}}$ & 0.2 & & & & & -0.1 & $\mathrm{C}$ & 0.6 \\
\hline 9 of 12 & & & & & & 12 of 15 & & \\
\hline 0.6 & & & & & & 0.3 & & \\
\hline
\end{tabular}

$\begin{array}{llll}\mathbf{5 . 2} & \mathbf{0 . 3} & 0.000911 & 27 \\ \mathbf{5 . 4} & \mathbf{0 . 3} & 0.000809 & 41 \\ \mathbf{5 . 3} & \mathbf{0 . 3} & 0.000786 & 22 \\ \mathbf{5 . 4} & \mathbf{0 . 3} & 0.000796 & 28 \\ \mathbf{5 . 3} & \mathbf{0 . 3} & 0.000808 & 39 \\ \mathbf{3 . 9} & \mathbf{0 . 3} & 0.000595 & 10 \\ \mathbf{4 . 9} & \mathbf{0 . 3} & & \end{array}$

$\begin{array}{ll}0.280439 & 41\end{array}$ $\begin{array}{ll}0.280454 & 36\end{array}$ $\begin{array}{ll}0.280444 & 38\end{array}$ $0.280454 \quad 44$

$\begin{array}{rrr}-\mathbf{0 . 6} & 1.5 & \mathbf{2 . 4} \\ \mathbf{0 . 7} & 1.3 & \mathbf{2 . 3} \\ \mathbf{0 . 0} & 1.3 & \mathbf{2 . 4} \\ \mathbf{0 . 1} & 1.6 & \mathbf{2 . 5} \\ \mathbf{0 . 0} & 1.3 & \mathbf{2 . 3} \\ \mathbf{- 0 . 3} & 1.0 & \mathbf{2 . 2}\end{array}$

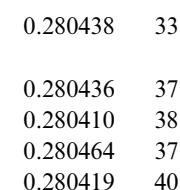
$\begin{array}{ll}0.280388 & 47 \\ 0.280372 & 43 \\ 0.280387 & 71 \\ 0.280382 & 43 \\ 0.280365 & 42 \\ 0.280438 & 65 \\ 0.280440 & 39 \\ 0.280383 & 36 \\ 0.280355 & 35 \\ 0.280311 & 44 \\ 0.280364 & 47 \\ 0.280387 & 38 \\ 0.280404 & 44 \\ 0.280358 & 53 \\ 0.280393 & 41 \\ 0.280369 & 41 \\ 0.280374 & 35 \\ 0.280385 & 42\end{array}$ (n) 
Table 1 (continued)

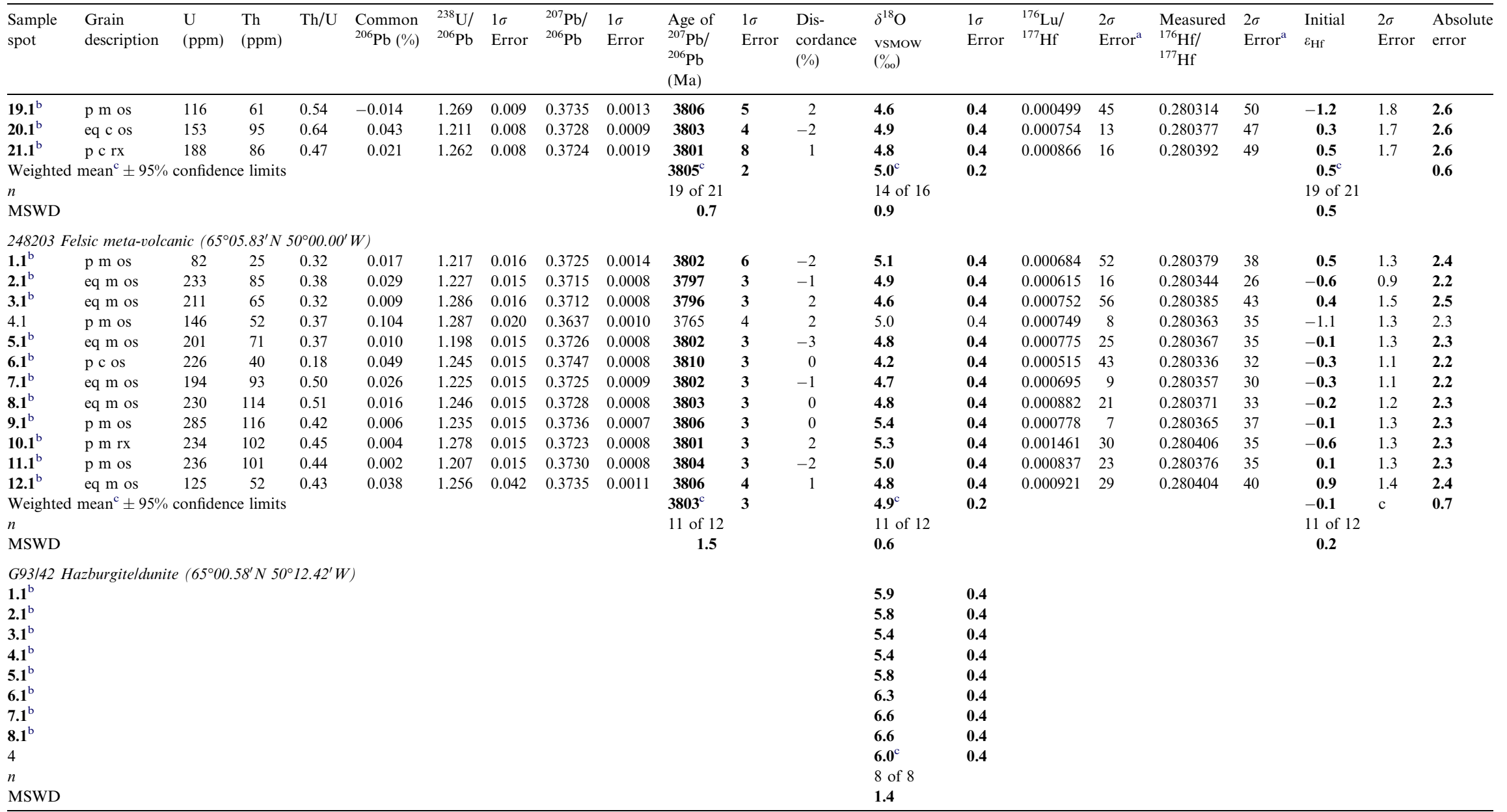

Grain descriptions: Habit: p, prismatic; ov, oval; eq, equant; an, anhedral.

Analysis site: c, core; m, middle; ed, edge.

Zonation: os, oscillatory; rx, recrystallized.

${ }^{\mathrm{a}} \times 10^{-6}$.

b Analysis used for weighted mean or mean calculations.

${ }^{c}$ Weighted mean $\pm 95 \%$ confidence limits.

${ }^{\mathrm{d}}$ Mean $\pm 1 \sigma$. 


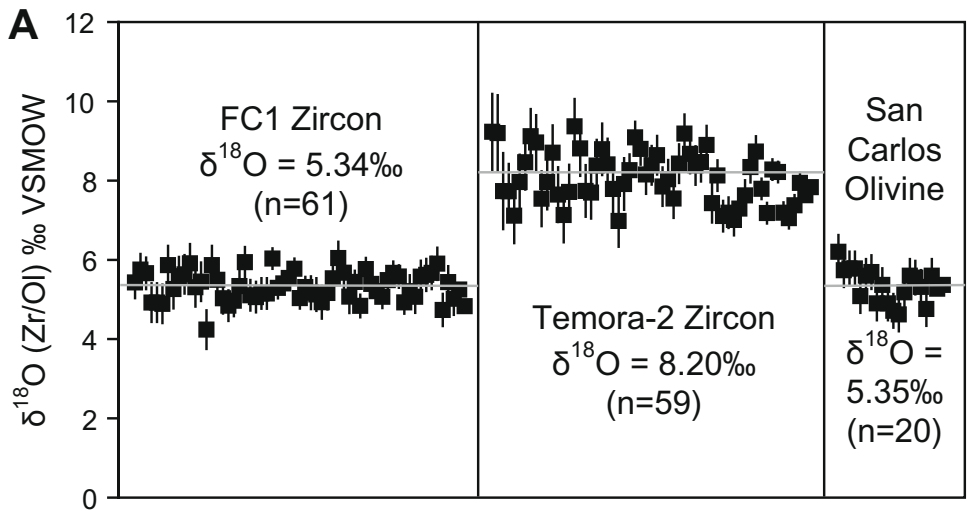

Zircon/Olivine Reference Material

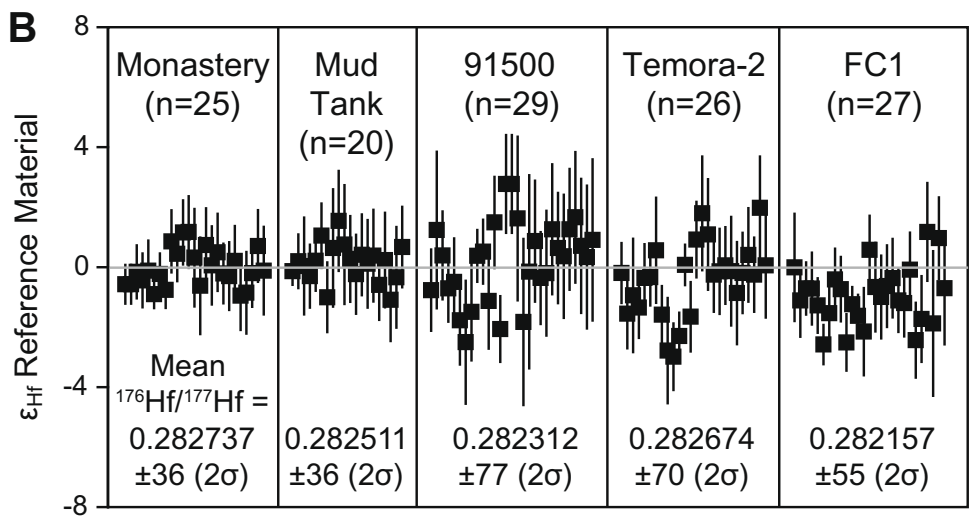

Zircon Reference Material

Fig. 4. (A) Spot-to-spot reproducibility of $\pm 1.0-0.3 \%$ during oxygen isotopic analysis of zircon and olivine reference material. (B) Zircon reference material ${ }^{176} \mathrm{Hf} /{ }^{177} \mathrm{Hf}$ ratios presented as $\varepsilon_{\mathrm{Hf}}$ deviations from values of Woodhead and Hergt (2005).

$3.9 \%$ to $5.4 \%$, with a mean of $4.9 \pm 0.5 \%$ ( $1 \sigma$, Fig. $6 \mathrm{~K})$. $\varepsilon_{\mathrm{Hf}(\mathrm{T})}$ values $(n=11)$ cluster at 0.7 to -0.7 epsilon units with a weighted mean of $0.0 \pm 0.7(95 \%$ c.l., $\mathrm{MSWD}=0.2$, Fig. 6L).

\subsection{Felsic volcanic samples 248202 and 248203}

Grains from 248202 and 248203 are small (typically $<100 \mu \mathrm{m}$ in length), prismatic, oval or equant in habit, with fine-scale oscillatory zoning cut by recrystallised domains. ${ }^{207} \mathrm{~Pb} /{ }^{206} \mathrm{~Pb}$ ages $(n=23)$ from the two samples range from 3811 to $3738 \mathrm{Ma}$, with an average $\mathrm{Th} / \mathrm{U}$ of 0.49 and weighted means of $3805 \pm 2$ and $3803 \pm 3 \mathrm{Ma}(\mathrm{MSWD}=0.7$ and 1.5, respectively, Fig. $6 \mathrm{M}$ and $\mathrm{P}$ ). A total of $28 \delta^{18} \mathrm{O}$ analyses range from $4.2 \%$ to $5.5 \%$, with indistinguishable weighted means of $5.0 \pm 0.2 \% \quad(\mathrm{MSWD}=0.9$, Fig. $6 \mathrm{~N})$ and $4.9 \pm 0.2 \%$ o (MSWD $=0.6$, Fig. 6Q). Initial $\varepsilon_{\mathrm{Hf}}$ values $(n=32)$ clustered tightly from 2.5 to -1.9 with weighted means of $0.5 \pm 0.6(\mathrm{MSWD}=0.5$, Fig. $6 \mathrm{O})$ and $-0.1 \pm 0.7$ $(\mathrm{MSWD}=0.2$, Fig. $6 \mathrm{R})$.

\subsection{G93/42}

Eight $\delta^{18} \mathrm{O}_{\mathrm{Ol}}$ analyses on $\mathrm{G} 93 / 42$ olivine (referenced to San Carlos olivine at 5.35\% ; Eiler et al., 2007) recorded a range of compositions from $5.4 \%$ to $6.6 \%$, with a weighted mean of $6.0 \pm 0.4 \%$ (MSWD $=1.4$, Fig. 7 ). A similar range of compositions has been measured within olivine from enriched mantle II (EM2) lavas $\left(\delta^{18} \mathrm{O}_{\mathrm{Ol}}=5.4-6.1 \%\right.$; Eiler et al., 1997b) interpreted as reflecting a minor component of recycled sediment in their source region, or metasomatism by sediment-derived fluids or melts (Eiler et al., 1997b).

A previous isotopic study of IGC ultramafic bodies south of the Isua supracrustal belt by Lowry et al. (2003) reported laser fluorination $\delta^{18} \mathrm{O}$ data for olivine and other mineral separates from the Ujarassuit Nûnat layered body and a nearby unlayered dunite body. Olivine analyses were normalized to a San Carlos olivine $\delta^{18} \mathrm{O}_{\mathrm{Ol}}$ composition of $4.88 \%$. With this normalization, olivine from a layered intrusion peridotite gave $\delta^{18} \mathrm{O}_{\mathrm{O} 1}$ values of $4.49-4.89 \%$, interpreted as representing late-magmatic re-equilibration resulting in small negative shifts in $\delta^{18} \mathrm{O}$. A massive, nonlayered dunite body had a higher olivine $\delta^{18} \mathrm{O}_{\mathrm{O} 1}$ value of $5.25 \%$, consistent with the mean of mantle peridotite olivine $(5.18 \pm 0.28 \%$, Mattey et al., 1994) or ocean island basalt olivine $(5.21 \pm 0.08 \%$, Eiler et al., 1995). If the ion probe ${ }^{18} \mathrm{O} /{ }^{16} \mathrm{O}$ analyses of $\mathrm{G} 93 / 42$ olivine from our study are renormalized to a San Carlos olivine $\delta^{18} \mathrm{O}_{\mathrm{Ol}}$ reference of $4.88 \%$ as used by Lowry et al. (2003), then they record a similar "mantle-like" $\delta^{18} \mathrm{O}_{\mathrm{Ol}}$ composition of $5.5 \pm 0.4 \%$. 

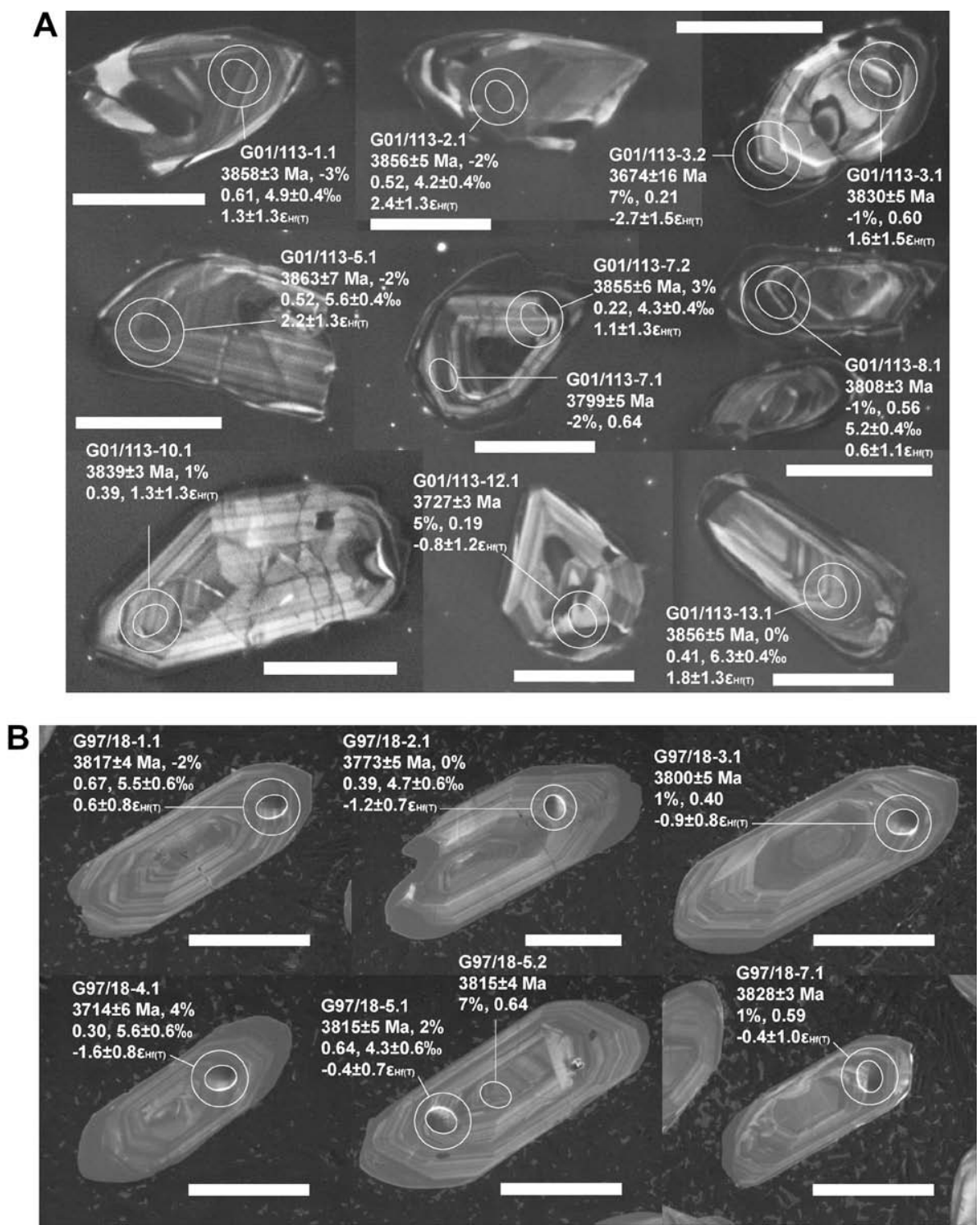

Fig. 5. Representative zircon CL images for samples (A) G01/113 and (B) G97/18 recording analysis locations, ${ }^{207} \mathrm{~Pb} /{ }^{206} \mathrm{~Pb}$ crystallisation ages, $\%$ discordance, $\mathrm{Th} / \mathrm{U}$ ratios, $\delta^{18} \mathrm{O}$ and $\varepsilon_{\mathrm{Hf}(\mathrm{T})}$ compositions. Scale bars are $100 \mu \mathrm{m}$.

\section{DISCUSSION}

\subsection{Considerations in Hf isotopic analysis}

Previous ${ }^{176} \mathrm{Hf}$ studies on Eoarchaean and Hadean zircon have covered samples sourced from ancient terranes on several continents and employed several different analytical techniques, evolving from bulk zircon analysis by IDTIMS (Patchett and Tatsumoto, 1980a), to single grain methods using ID-ICPMS (Blichert-Toft et al., 1997) to sub-grain methods using laser ablation MC-ICPMS (Harrison et al., 2005; Iizuka and Hirata, 2005), that can be combined with in situ U-Pb dating obtained by using large ion probes. Lu-Hf isotopic studies of Archaean and Hadean zircon include detrital grains of the Jack Hills meta-conglomerate from the Narryer Terrane, Yilgarn Craton, Western
Australia (Amelin et al., 1999; Harrison et al., 2005, 2008; Blichert-Toft and Albarède, 2008), the Acasta Gneiss Complex, Western Slave Province, Northwest Territories, Canada (Amelin et al., 1999, 2000), the Napier Complex, Enderby Land, East Antarctic Craton (Choi et al., 2006), Barberton Mountain Land, Kaapvaal Craton, South Africa (Amelin et al., 2000), the Eastern Pilbara Terrane, Pilbara Craton, Western Australia (Amelin et al., 2000), and the Itsaq Gneiss Complex, as studied here (Pettingill and Patchett, 1981; Vervoort et al., 1996; Vervoort and Blichert-Toft, 1999; Amelin et al., 2000; Kemp et al., 2009).

We have adopted the CHUR reference parameters of Bouvier et al. (2008). In contrast to uncertainties in CHUR estimates, which have little affect on calculated initial $\varepsilon_{\mathrm{Hf}}$ for Eoarchaean and Hadean rocks, a generally accepted recent revision of the $\lambda^{176} \mathrm{Lu}$ decay constant has required 
significant re-interpretation of $\mathrm{Hf}$ data from ancient rocks (Bennett, 2003; Amelin and Davis, 2005; Albarède et al., 2006). From 1980 to 2001, Lu-Hf isotopic studies routinely used values for $\lambda^{176} \mathrm{Lu}$ ranging from 1.93 to $1.98 \times 10^{-11} \mathrm{yr}^{-1}$ as determined from physical counting experiments (e.g. Sguigna et al., 1982) and meteorite isochrons (e.g. Patchett and Tatsumoto, 1980b; Bizzarro et al., 2003). However, new work on ancient terrestrial, high $\mathrm{Lu} / \mathrm{Hf}$ minerals and rocks dated by $\mathrm{U}-\mathrm{Pb}$ and $\mathrm{Lu}-\mathrm{Hf}$ methods suggest a mean $\lambda^{176} \mathrm{Lu}$ of $1.867 \pm 13 \times 10^{-11} \mathrm{yr}^{-1}$, revealing a $\sim 4 \%$ dichotomy between meteorite and terrestrial datasets (Scherer et al., 2001; Söderlund et al., 2004). The new values derived from terrestrial samples have been supported by $\mathrm{Lu}-\mathrm{Hf}$ and $\mathrm{U}-\mathrm{Pb}$ isochrons from phosphate minerals contained within two largely unaltered and rapidly cooled meteorites, providing $\lambda^{176} \mathrm{Lu}$ of 1.864 and $1.832 \times 10^{-11} \mathrm{yr}^{-1}$ (Amelin, 2005). As with other recent $\mathrm{Hf}$ isotopic studies, we have adopted the $\lambda^{176} \mathrm{Lu}$ decay constant of Scherer et al. (2001) and Söderlund et al. (2004). The $4 \%$ change in decay constant has a progressive effect on the calculated initial $\varepsilon_{\mathrm{Hf}}$ with increasing age, which results in large effects on low $\mathrm{Lu} / \mathrm{Hf}$ rocks and minerals older than $3.6 \mathrm{Ga}$, such as the zircon analysed here. Use of the higher ${ }^{176} \mathrm{Lu}$ decay constant would result in an apparent increase in initial $\varepsilon_{\mathrm{Hf}}$ of 3-4 units, such that the IGC samples (with the exception of G01/113) would have $\varepsilon_{\mathrm{Hf}(\mathrm{t})}=+3$ to +4 and appear to be from a depleted mantle source with long-term high $\mathrm{Lu} / \mathrm{Hf}$, rather than from a mantle with chondritic Lu/Hf.

\subsection{Insights to TTG petrogenesis from Greenland zircon}

$\varepsilon_{\mathrm{Hf}(\mathrm{T})}$

Zircon grains from samples G01/113, G97/18 and 248228 experienced only minor late recrystallisation, and their host rocks do not contain melt segregations or veins (Nutman et al., 1999, 2000, 2007b). $\varepsilon_{\mathrm{Hf}(\mathrm{T})}$ data from the igneous population in these samples plot as simple clusters representing the rock's crystallisation age with near chondritic initial ${ }^{176} \mathrm{Hf} /{ }^{177} \mathrm{Hf}$ compositions (Fig. 6F, I, and L). Samples G01/113 and G97/18 additionally record late minor recrystallisation or lead loss events, with more negative initial $\varepsilon_{\mathrm{Hf}}$ values that track single linear arrays away from the main population. In contrast, sample G01/36 has evidence of melt segregation through the tonalite matrix in the form of fine-scale veining. This coincides with younger, locally pronounced recrystallisation and overgrowth of the protolith magmatic zircon, noted from CL-guided in situ U-Pb dating (Nutman et al., 2007a; Electronic Annex EA-8). This lithological complexity appears to be reflected in the heterogeneity of the Hf isotopic data for G01/36. This may reflect the growth of new zircon during later thermal events, incorporating a mixture of Hf components from within the rock matrix as well as extraneous sources from outside the rock system, introduced by fluids or melts that may be isotopically diverse. Consequently, the different field geological and zircon CL petrography characteristics for samples G01/36 versus samples G01/113, G97/ 18 and 248228 are reflected in their contrasting zircon Hf isotopic systematics.
The slope of each zircon array from samples G01/36, $\mathrm{G} 01 / 113$ and $\mathrm{G} 97 / 18$ define ${ }^{176} \mathrm{Lu} /{ }^{177} \mathrm{Hf}$ ratios of 0.007 , 0.009 and 0.012 , respectively (Fig. 6C, F and I). Such ratios are typically interpreted to reflect the ${ }^{176} \mathrm{Lu} /{ }^{177} \mathrm{Hf}$ of the melt source reservoir that the zircons crystallised from (e.g. Griffin et al., 2004; Kemp et al., 2006), and are actually similar to that of modern mature continental crust $(0.013-$ 0.014; Taylor and McLennan, 1995) and Precambrian granitoids (0.009; Vervoort and Patchett, 1996). For these tonalites, however, ${ }^{176} \mathrm{Lu} /{ }^{177} \mathrm{Hf}$ ratios of the bulk rock have been independently determined by measuring the trace element concentrations of powdered whole rock samples (Nutman et al., 1999, 2007b) yielding lower values of $\sim 0.0017$, consistent with those typical of HREE depleted TTG magmas (Vervoort and Blichert-Toft, 1999; Blichert-Toft and Albarède, 2008). Additionally, the ${ }^{176} \mathrm{Lu} /{ }^{177} \mathrm{Hf}$ ratios of zircon from these samples $(\sim 0.0005)$ were measured during LA-MC-ICPMS analysis (Electronic Annex EA-7). These arrays have been interpreted as the products of recrystallisation and subtle $\mathrm{Pb}$-loss, where initial $\varepsilon_{\mathrm{Hf}}$ values are calculated with younger ages. While $\mathrm{Hf}$ was incorporated into the grain during igneous crystallisation, the $\mathrm{U}-\mathrm{Pb}$ ages were partially reset during subsequent thermal events, resulting in progressively negative calculations of $\varepsilon_{\mathrm{Hf}}$ at this given age (e.g. Kemp et al., 2009).

With the exception of sample G01/113 at $1.3 \pm 0.7 \varepsilon_{\mathrm{Hf}(\mathrm{T})}$ units, weighted mean initial $\varepsilon_{\mathrm{Hf}}$ values for the zircons for each tonalite and felsic volcanic sample range from $0.5 \pm 0.6$ to $-0.1 \pm 0.7 \varepsilon_{\mathrm{Hf}}$ units, all plotting within error of the CHUR reference line (Fig. 6C, F, I, L, O and R). This requires that the ultimate upper mantle source of these magmas had a time-averaged chondritic Lu/Hf ratio. The interpretation that this is a mantle source feature, rather than an artifact of an intermediate stage of evolution in a high $\mathrm{Lu} / \mathrm{Hf}$ crustal environment (e.g. Kemp et al. 2009) is based on the complete lack of geochemical evidence for older crustal components in these rocks, and the assumption that these tonalitic magmas did not form directly from the mantle but required an intermediate mafic stage (Barker and Arth, 1976; Martin, 1986; Drummond and Defant, 1990). The timescale of this intermediate stage is poorly constrained by $\mathrm{Nd}$ and $\mathrm{Hf}$ isotopic data as most mafic magmas, such as those that produce oceanic crust, have $\mathrm{Lu} / \mathrm{Hf}$ and $\mathrm{Sm} / \mathrm{Nd}$ ratios similar to that of their upper mantle sources. Thus substantial differences in the $\mathrm{Hf}$ and $\mathrm{Nd}$ isotopic compositions of oceanic crust as compared to their upper mantle source may not be generated on tens to hundreds of million year time scales, because such mafic rocks do not rapidly evolve away from chondritic $\varepsilon_{\mathrm{Hf}(\mathrm{T})}$. Although it would be possible to produce magmas of apparent chondritic composition by the fortuitous mixing of melts derived from both depleted mantle (high $\varepsilon_{\mathrm{Hf}(\mathrm{T})}$ ) and ancient enriched crustal reservoirs (low $\left.\varepsilon_{\mathrm{Hf}(\mathrm{T})}\right)$ this would probably result in a broad range of positive to negative initial $\varepsilon_{\mathrm{Hf}}$, in contrast to the consistently chondritic compositions observed in the tonalites produced over a 180 million year period.

Additionally, the source of the tonalites must not only have had a chondritic ${ }^{176} \mathrm{Lu} /{ }^{177} \mathrm{Hf}$ ratio from 4.56 to $3.88 \mathrm{Ga}$ (the age of the oldest tonalite) but must also have retained this chondritic composition for at least the next 

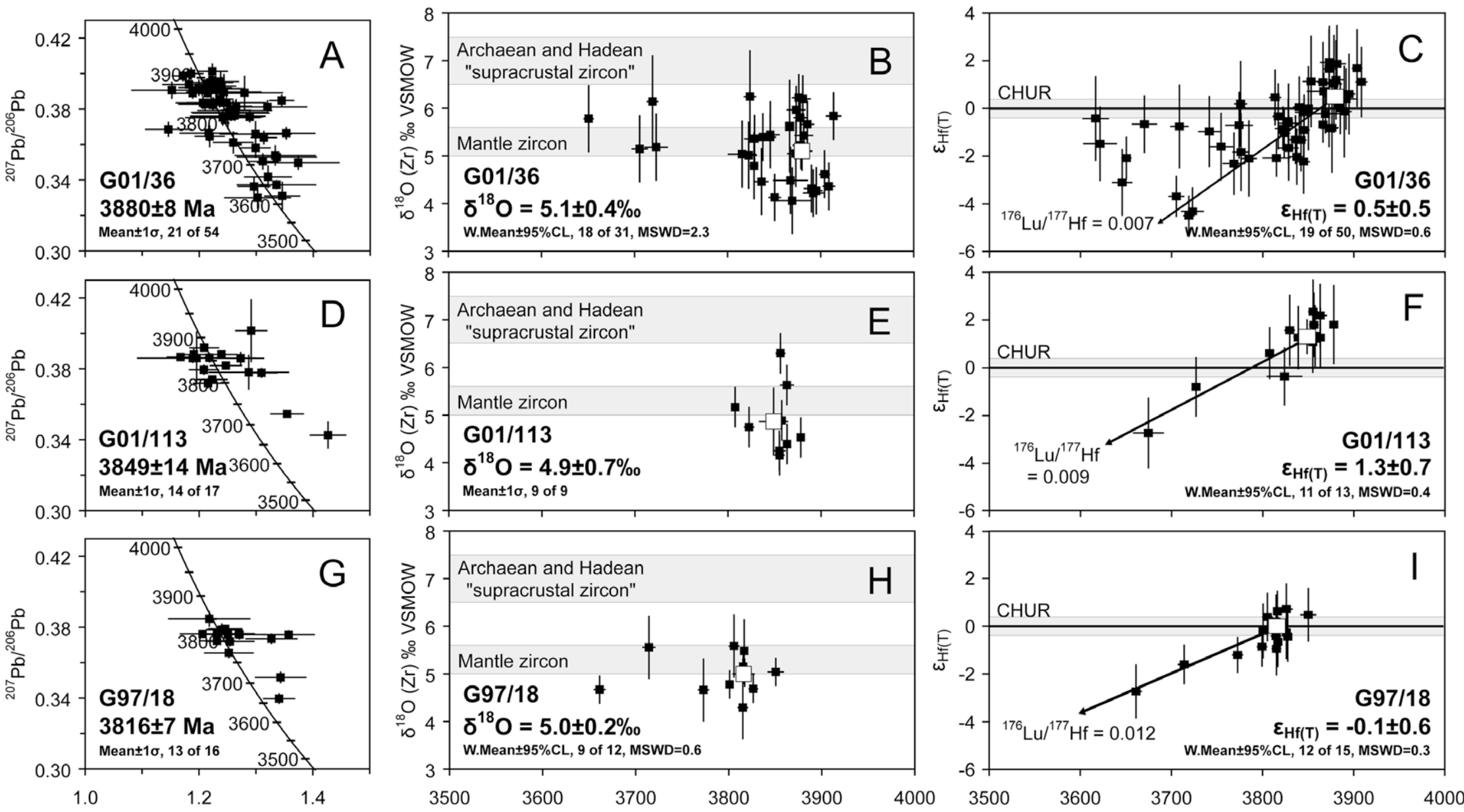

Fig. 6. Terra-Wasserburg diagrams and plots of $\delta^{18} \mathrm{O}$ and $\varepsilon_{\mathrm{Hf}(\mathrm{T})}$ against corresponding ${ }^{207} \mathrm{~Pb} /{ }^{206} \mathrm{~Pb}$ crystallisation ages for zircon analysis. Terra-Wasserburg data-point error crosses are at the $2 \sigma$ level. $\delta^{18} \mathrm{O}$ and $\varepsilon_{\mathrm{Hf}(\mathrm{T})}$ uncertainties are $1 \sigma$ and $2 \sigma$, respectively, while ${ }^{207} \mathrm{~Pb} /{ }^{206} \mathrm{~Pb}$ ages are at $1 \sigma$ level. Weighted mean ${ }^{207} \mathrm{~Pb} /{ }^{206} \mathrm{~Pb}, \delta^{18} \mathrm{O}$ and $\varepsilon_{\mathrm{Hf}(\mathrm{T})} \mathrm{values}$ for the igneous populations of each sample are represented as white squares. Fields for mantle zircon, Archaean-Hadean "supracrustal zircon" and CHUR from Valley et al. (1998), Cavosie et al. (2005a) and Bouvier et al. (2008). 

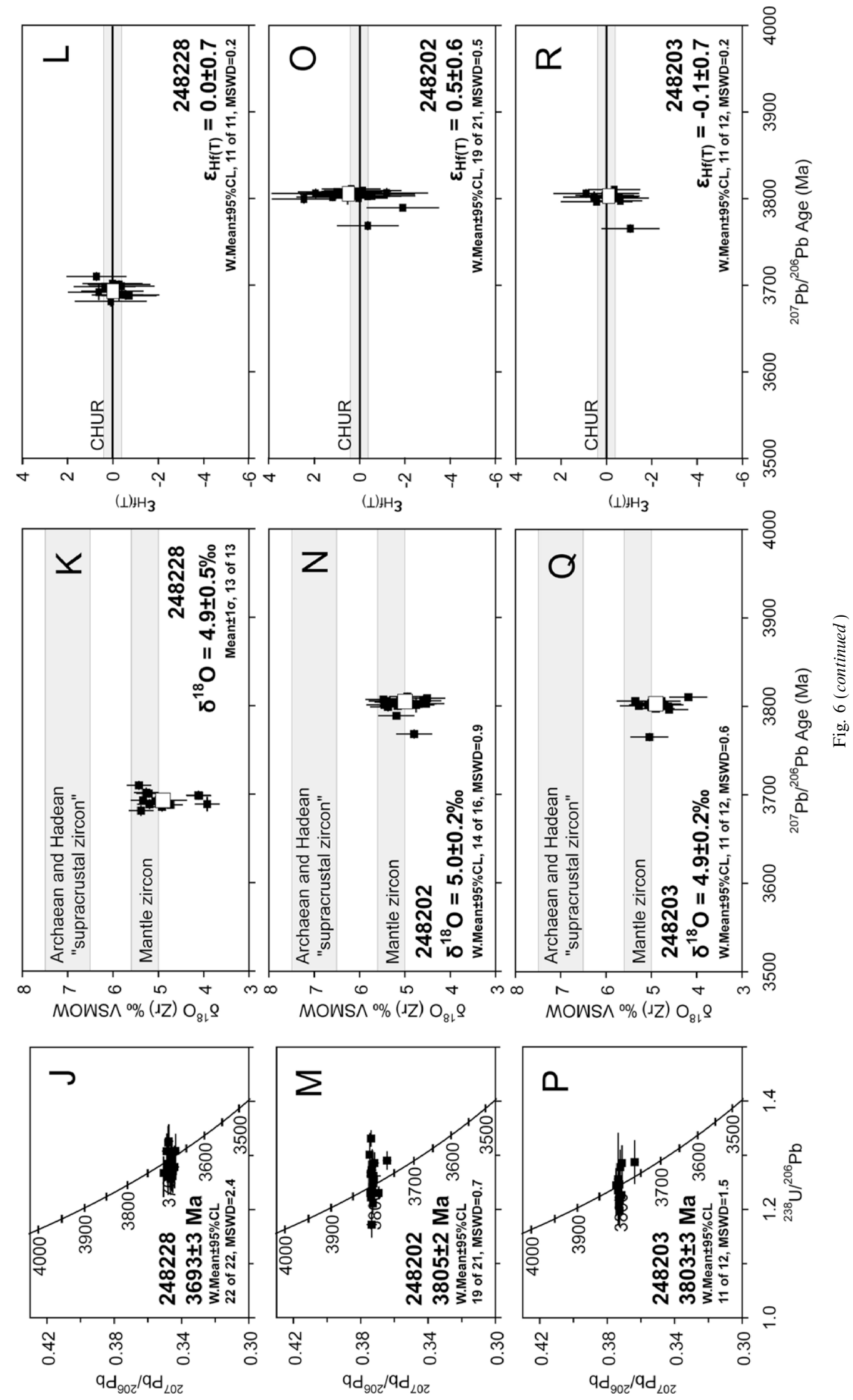


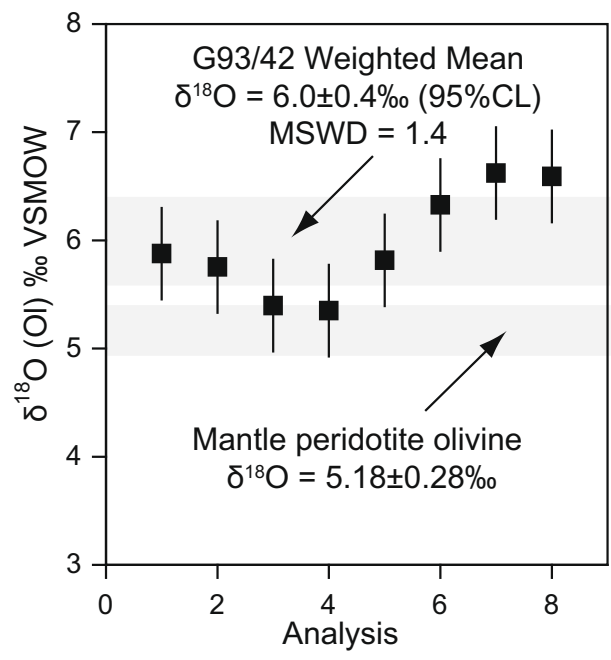

Fig. 7. $\delta^{18} \mathrm{O}$ compositions of $\mathrm{G} 93 / 42$ olivine with field for mantle peridotite olivine from Mattey et al. (1994).
180 million years, as the signature is recorded by samples ranging in age from 3.88 to $3.69 \mathrm{Ga}$. This observation, based on Lu-Hf data from Archaean zircons, implies that there was not a voluminous continent-forming, or mantle differentiation episode leading to $\mathrm{Lu} / \mathrm{Hf}$ fractionation at $\sim 3.9 \mathrm{Ga}$, associated, for example, with a period of heavy meteorite bombardment such as the postulated coeval lunar "terminal cataclysm" (Tera et al., 1974; Ryder, 2002). The MORB source mantle today has a high $\mathrm{Lu} / \mathrm{Hf}$ ratio $(=0.369)$ largely resulting from the extraction of low $\mathrm{Lu} /$ Hf continental crust (e.g. Workman and Hert, 2005). If the upper mantle source of the IGC tonalites became similarly depleted at $3.9 \mathrm{Ga}$, then by $3.7 \mathrm{Ga}$ it would have evolved to $\varepsilon_{\mathrm{Hf}(\mathrm{T})}=+2$. Values this positive are not seen in the Hf data presented here.

This evidence for a chondritic $\mathrm{Lu} / \mathrm{Hf}$ isotopic composition in the source of Archaean continents is in contrast to previous interpretation from $\mathrm{Hf}$ isotopic studies of the IGC (Vervoort et al., 1996; Vervoort and Blichert-Toft, 1999; Amelin et al., 2000), which suggested that these rocks were
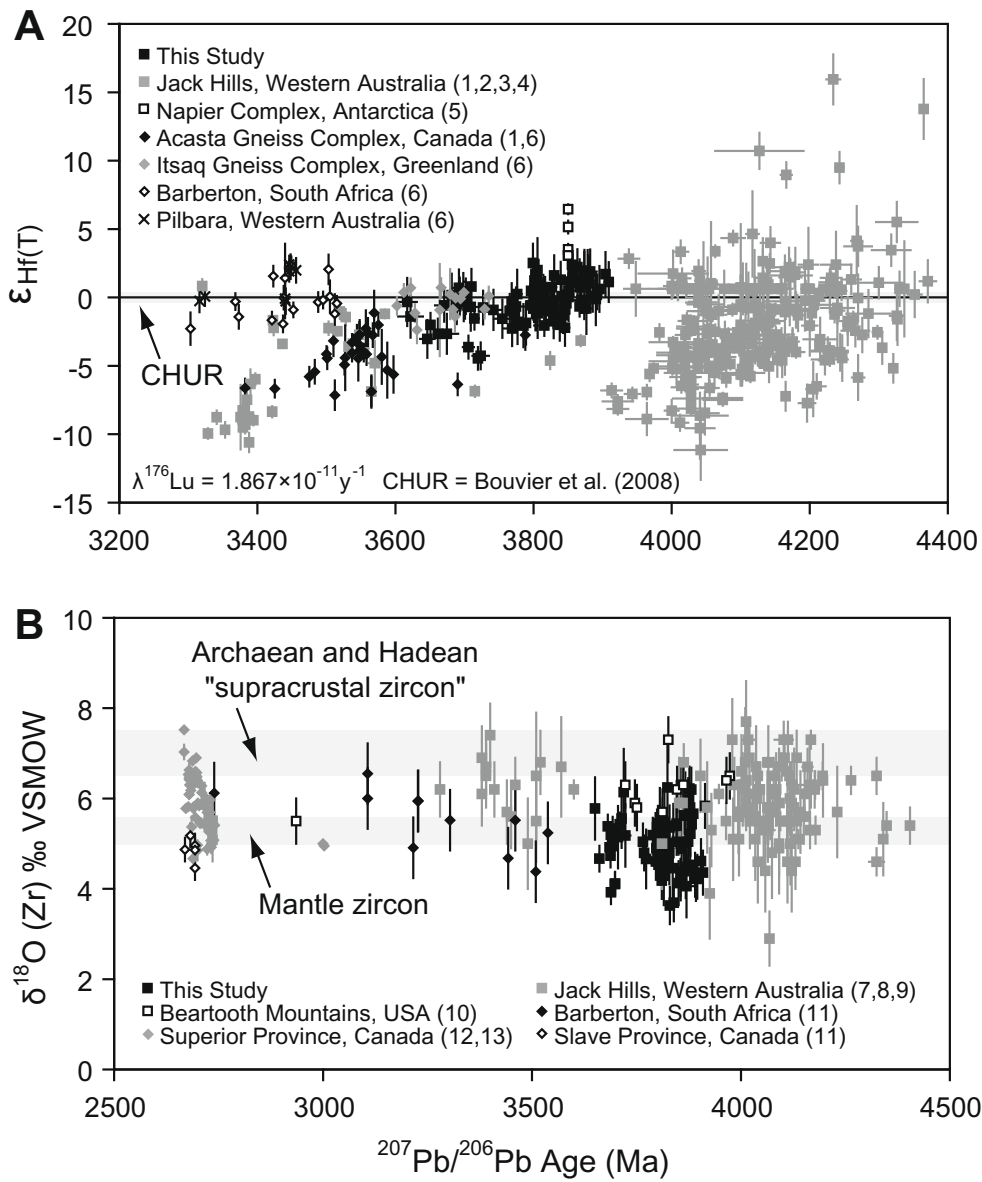

Fig. 8. (A) Single zircon $\varepsilon_{\mathrm{Hf}(\mathrm{T})}$ data with ${ }^{207} \mathrm{~Pb} /{ }^{206} \mathrm{~Pb}$ ages from the Hadean to Paleoarchaean. All measured ${ }^{176} \mathrm{Hf} /{ }^{177} \mathrm{Hf}$ ratios were recalculated to $\varepsilon_{\mathrm{Hf}(\mathrm{T})}$ with CHUR parameters from Bouvier et al. (2008) and $\lambda^{176} \mathrm{Lu}$ of $1.867 \times 10^{-11} \mathrm{yr}^{-1}$ from Scherer et al. (2001) and Söderlund et al. (2004). (B) $\delta^{18} \mathrm{O}$ compositions of dated Hadean to Archaean zircon. Field for mantle zircon from Valley et al. (1998) and Archaean-Hadean "supracrustal zircon" from Cavosie et al. (2005a). Data sourced from: (1) Amelin et al. (1999), (2) Harrison et al. (2005), (3) Harrison et al. (2008), (4) Blichert-Toft and Albarède (2008), (5) Choi et al. (2006), (6) Amelin et al. (2000), (7) Peck et al. (2001), (8) Cavosie et al. (2005), (9) Trail et al. (2007), (10) Valley et al. (2005), (11) King (2001), (12) King et al. (1998), and (13) King et al. (2000). 
derived from melting of a depleted upper mantle reservoir that had a long-term, high ${ }^{176} \mathrm{Lu} /{ }^{177} \mathrm{Hf}$ ratio. While datasets from this and previous studies yield similar results when the data are recalculated using the same CHUR parameters and ${ }^{176} \mathrm{Lu}$ decay constants, this reinterpretation mainly reflects recent revision to the ${ }^{176} \mathrm{Lu}$ decay constant used in $\varepsilon_{\mathrm{Hf}(\mathrm{T})}$ calculations, and also the improvement of analytical methods facilitating combined $\mathrm{U}-\mathrm{Pb}$ and $\mathrm{Lu} / \mathrm{Hf}$ analysis on portions of single zircon grains. In order to examine the broader context of these and previous results on Eoarchaean and Hadean zircon, Fig. 8A compiles published single-grain $\varepsilon_{\mathrm{Hf}(\mathrm{T})}$ analyses, recalculated with CHUR parameters from Bouvier et al. (2008) and $\lambda^{176} \mathrm{Lu}$ of $1.867 \times 10^{-11} \mathrm{yr}^{-1}$ from Scherer et al. (2001) and Söderlund et al. (2004).

Recent studies on the Jack Hills zircon initially reported both highly positive and negative $\varepsilon_{\mathrm{Hf}(\mathrm{T})}$ values (Harrison et al., 2005; Blichert-Toft and Albarède, 2008) suggesting that the Hadean mantle experienced an early and widespread differentiation. However, a follow-up study then failed to detect further positive results (Harrison et al., 2008), suggesting consistency with the earlier interpretation of Amelin et al. (1999) that the Hadean zircons formed by the remelting of older crustal components. A similar petrogenesis is envisaged for zircon from the Acasta Gneiss Complex (Amelin et al., 1999, 2000). Distinct positive $\varepsilon_{\mathrm{Hf}(\mathrm{T})}$ values were measured during single-grain solution analyses of Gage Ridge zircon from the Napier Complex, Antarctica (Choi et al., 2006). However, the grains analysed were highly discordant and have complex age structures, potentially complicating this information. In contrast, samples from other Eoarchaean areas including the Itsaq Gneiss Complex, Barberton and Pilbara (Amelin et al., 2000) are dominated by near-chondritic compositions in the oldest grains from each suite. All datasets trend towards dominant proportions of negative $\varepsilon_{\mathrm{Hf}(\mathrm{T})}$ values in younger analyses, indicating an important shift to processes of crustal remelting and differentiation in the evolution of each complex.

\subsection{The oxygen isotopic composition of Eoarchaean mantle, sedimentary and oceanic reservoirs}

To investigate the significance of $\delta^{18} \mathrm{O}$ signatures of Eoarchaean granitoids, it is necessary to consider the $\delta^{18} \mathrm{O}$ compositions of components that may have contributed to their formation. Various lines of evidence support the concept that the Earth's mantle has not experienced a secular evolution of $\delta^{18} \mathrm{O}$. These include oxygen mass balance calculations for the growth and evolution of the continental crust (Valley et al., 2005), which demonstrate that the Earth's oxygen budget is overwhelmingly held by the mantle. Also direct $\delta^{18} \mathrm{O}$ measurements of Archaean materials including $2.7 \mathrm{Ga}$ zircon from Superior Province TTG magmas (King et al., 1998), 3.2 Ga olivine inclusions contained within syngenetic diamonds (Mattey et al., 1994) and $\sim 3.8 \mathrm{Ga}$ olivine from an unlayered dunite (Lowery et al., 2003) all pointing to the consistency of mantle compositions over time.

The new olivine data from sample G93/42, taken to represent the best estimate of a variably depleted $\sim 3.8 \mathrm{Ga}$ upper mantle composition, are broadly consistent with these results (Fig. 7). This further demonstrates that the ultimate mantle source of Eoarchaean tonalites was not significantly different from that of the present day. While modern mantle peridotite and ocean island basalt olivine record $\delta^{18} \mathrm{O}_{\mathrm{Ol}}$ compositions from $5.0 \%$ to $5.4 \%$ (Mattey et al., 1994; Eiler et al., 1995), G93/42 olivine $\left(\delta^{18} \mathrm{O}_{\mathrm{Ol}}\right.$ range $=5.4-6.6 \%$, weighted mean $=6.0 \pm 0.4 \%$ ) covers a similar range of compositions to that of EM2 lavas $\left(\delta^{18} \mathrm{O}_{\mathrm{Ol}}=5.4-6.1 \%\right.$; Eiler et al., 1997b) and may indicate that the Eoarchaean upper mantle was variably re-enriched in ${ }^{18} \mathrm{O}$ during magmatism from a minor contribution of recycled sediment, or by metasomatism from sediment derived fluids or melts. Alternatively the composition of G93/42 olivine may reflect minor alteration in response to tectonothermal events later during the Archaean.

Despite the fragmentation of Earth's sedimentary rock record, particularly through the Archaean, it is possible to resolve that the relative proportion of different types of sedimentary rocks have changed through time (Veizer and Mackenzie, 2003). Archaean sediments are dominated by greenstone belt sequences which record lower average $\delta^{18} \mathrm{O}_{\mathrm{WR}}$ values $(9-12 \%$; Longstaffe and Schwarcz, 1977; Lowe, 1994) as compared to Phanerozoic clastic sequences $(10-20 \%)$, pelagic clays $(15-25 \%)$, carbonates $(25-32 \%)$ and siliceous oozes $(35-42 \%$; Kolodny and Epstein, 1976). Owing to their less extreme compositional range, Archaean sediments would in theory be less efficient at shifting the $\delta^{18} \mathrm{O}_{\mathrm{WR}}$ of a primitive magma to more positive values as compared to some modern sediments. However, their ability to generate positive shifts of $\delta^{18} \mathrm{O}_{\mathrm{WR}}$ in Archaean and Hadean magmas is widely observed by $\delta^{18} \mathrm{O}$ zircon compositions of up to $7.5 \%$ in a minority of detrital grains from the Jack Hills (Peck et al., 2001; Wilde et al., 2001; Cavosie et al., 2005a; Trail et al., 2007) and the Beartooth Mountains (Valley et al., 2005; Fig. 8B).

Secular shifts in the $\delta^{18} \mathrm{O}$ of the Earth's oceans have also been debated (Walker and Lohmann, 1989; Wallmann, 2001; Knauth and Lowe, 2003; Kasting et al., 2006). The oceanic reservoir with a $\delta^{18} \mathrm{O}$ of $\sim 0 \%$ (e.g. Muehlenbachs, 1998 ) is buffered by mid ocean ridge hydrothermal circulation (Gregory and Taylor, 1981). While small variations on the order of $\pm 1 \%$ would have probably occurred in response to continental glaciation over geological time, significant shifts are not consistent with the regularity of $\delta^{18} \mathrm{O}_{\mathrm{WR}}$ recorded in ophiolite sequences through the geological record (Gregory and Taylor, 1981; Muehlenbachs, 1986, 1998).

\subsection{Insights to Eoarchaean tonalite petrogenesis from $\delta^{18} \mathrm{O}$}

The $\delta^{18} \mathrm{O}$ compositions of zircon from all samples range from $6.3 \%$ to $3.9 \%$ and straddle the field for mantle zircon (Fig. 6B, E, H, K, N and Q). Samples G01/36 and G97/18 show no indication of $\delta^{18} \mathrm{O}_{\mathrm{Zr}}$ disturbance associated with recrystallisation as indicated by the consistency of isotopic compositions, even in grains with lower ${ }^{207} \mathrm{~Pb} /{ }^{206} \mathrm{~Pb}$ ages (Fig. 6B and $\mathrm{H}$ ). The restricted range of $\delta^{18} \mathrm{O}_{\mathrm{Zr}}$ observed in the Eoarchaean zircon samples analysed spanning the interval 3.88-3.69 Ga is in accord with a global compilation of $\delta^{18} \mathrm{O}_{\mathrm{Zr}}$ from 1200 rocks spanning most of Earth's geolog- 
ical record, with samples from the Hadean and Archaean exhibiting a restricted range of zircon compositions from $\sim 4.0 \%$ to $7.5 \%$ (Valley et al., 2005; Fig. 8B). Specifically, zircon compositions from this study are consistent with those of other Archaean TTG suites from Barberton (King, 2001), the Superior Province (King et al., 1998, 2000) and the Slave Province (King, 2001).

Significantly, no samples from the IGC plot within the field defined by Archaean and Hadean "supracrustal zircon" $\left(\delta^{18} \mathrm{O}_{\mathrm{Zr}}=6.5-7.5 \%\right.$; Fig. 6 of Cavosie et al., 2005a). The simplest explanation for this feature of the dataset is that the sources of the tonalitic and felsic volcanic magmas had negligible amounts of supracrustal material that had experienced low-temperature to moderate-temperature water interaction. This suggests that ${ }^{18} \mathrm{O}$-enriched pelagic sediments or weathered continental crust were not involved or were insignificant in the production of these Archaean magmas. Similarly, pillow basalt or sheeted dike components from shallow hydrothermally altered oceanic crust with $\delta^{18} \mathrm{O}_{\mathrm{WR}}$ values from $7 \%$ to $15 \%$ (Gregory and Taylor, 1981; Alt et al., 1986) were not a significant source. Such values are relevant to the Eoarchaean, as shown by $\delta^{18} \mathrm{O}_{\mathrm{WR}}$ values of $6.5-9.9 \%$ for $\sim 3.7 \mathrm{Ga}$ amphibolitised pillow basalts from the Isua supracrustal belt (Furnes et al., 2007). This new, first-order observation suggests that if oceanic crust was the mafic source of the TTG magmas, a pelagic sediment drape and/or the uppermost section of basaltic crust either dewatered sub-solidus during shallow subduction, or was scraped off the rest of the subducted or buried slab. This has implications for the structure of ancient convergent plate boundaries. If the upper section of the oceanic crust is scraped-off via a decollement along a structural weakness within the relatively warm and young mafic crustal section, it might form a fold and thrust belt near the plate boundary. Weathering and erosion of such rocks would produce mafic pelitic sediments, which could then be found interlayered with intermediate to felsic volcanic rocks derived from arc-like sources. Supporting this, within the Isua supracrustal belt there are prominent units of such rocks interpreted as being derived from weathered mafic rocks (Nutman et al., 1984; Bolhar et al., 2005) that are interlayered with felsic volcanic rocks dated at $\sim 3.7$ Ga (Nutman et al., 1996, 1997). Alternative explanations for this feature could be that any ${ }^{18} \mathrm{O}$-enriched components that failed to be removed from the down-going slab were not involved during melting by some unknown selection process, that the volume of contaminants was not significant enough to shift the composition of the magma to more positive values, or that ${ }^{18} \mathrm{O}$ enriched and ${ }^{18} \mathrm{O}$-depleted contaminants were always present in equilibrium proportions such as to average out the mean composition of the mafic crust.

A more precise characterization of each sample's $\delta^{18} \mathrm{O}_{\mathrm{Zr}}$ value is given by their means and weighted means, which lie in a narrow range from $4.9 \pm 0.7 \%$ to $5.1 \pm 0.4 \%$ (Fig. $6 \mathrm{~B}$, $\mathrm{E}, \mathrm{H}, \mathrm{K}, \mathrm{N}$ and $\mathrm{Q}$ ). These averages all lie within error, but towards the lower limit of the field defined for zircon derived from the mantle or melts equilibrated at similar temperatures $\left(\delta^{18} \mathrm{O}_{\mathrm{Zr}}=5.3 \pm 0.3 \%\right.$, Valley et al., 1998). This suggests that the tonalite and felsic volcanic magmas were largely formed by the melting of an unaltered or weakly hydrated gabbroic source. The average $\delta^{18} \mathrm{O}_{\mathrm{WR}}$ for such lithologies is $5.5 \pm 0.2 \%$ (Eiler, 2001) and would theoretically crystallise zircon with a $\delta^{18} \mathrm{O}_{\mathrm{Zr}}$ of $5.3 \%$. The subtly lower average $\delta^{18} \mathrm{O}_{\mathrm{Zr}}$ compositions of $4.9-5.1 \%$ recorded by each IGC sample may reflect a systematic component of ${ }^{18} \mathrm{O}$-depleted material within the mafic source to the TTG magmas.

To investigate this, let us consider a juvenile tonalitic sample with $70 \mathrm{wt} \% \mathrm{SiO}_{2}$ and $\delta^{18} \mathrm{O}_{\mathrm{Zr}}$ of $5.3 \%$. This sample would have a $\delta^{18} \mathrm{O}_{\mathrm{WR}}$ of $7.1 \%$, based on zircon - whole rock fractionation of Valley et al. (1994, 2005). Such a tonalite melt would be solely generated by the partial melting of an unaltered mafic source with $\sim 50 \mathrm{wt} \% \quad \mathrm{SiO}_{2}$ and $\delta^{18} \mathrm{O}_{\mathrm{WR}}$ of $5.5 \%$. The $1.6 \%$ shift between $\delta^{18} \mathrm{O}_{\mathrm{WR}}$ compositions is due to the contrasting $\mathrm{SiO}_{2}$ contents of the magmas, with the tonalite containing a larger modal proportion of higher $\delta^{18} \mathrm{O}$ phases (e.g. quartz and feldspar). The tonalite $\delta^{18} \mathrm{O}_{\mathrm{Zr}}$ and mafic rock $\delta^{18} \mathrm{O}_{\mathrm{WR}}$ values remain relatively constant as zircon - whole rock fractionation increases at nearly identical rates to $\delta^{18} \mathrm{O}_{\mathrm{WR}}$ (Valley et al., 2003). The average $\delta^{18} \mathrm{O}_{\mathrm{Zr}}$ of $5.0 \%$ recorded across all four tonalite samples can be used to calculate an estimate of the contribution of ${ }^{18} \mathrm{O}$-depleted crust that could mix with an unaltered mafic crust $\left(\delta^{18} \mathrm{O}_{\mathrm{WR}}=5.5 \%\right)$ to produce a tonalite melt with $\delta^{18} \mathrm{O}_{\mathrm{WR}}$ of $6.8 \%$. The ${ }^{18} \mathrm{O}$-depleted component is assumed to be a high-temperature altered, cumulate gabbro with $\delta^{18} \mathrm{O}_{\mathrm{WR}}$ of $4.0 \%$ (Gregory and Taylor, 1981; Staudigel et al., 1995) as this is the most volumetrically significant low- $\delta^{18} \mathrm{O}$ component in oceanic crustal sections as represented by ophiolites. By mass balance the ${ }^{18} \mathrm{O}$-depleted material would constitute a maximum of $\sim 20 \%$ of the source mafic rock which produced the tonalite magma. Thus the $\delta^{18} \mathrm{O}_{\mathrm{Zr}}$ values slightly lower than mantle values may indicate a volumetrically minor component of subducted, altered oceanic crust, is involved in the genesis of the TTG. This would also be in accord with the general consensus from experimental petrology (e.g. Rapp et al., 1991; Rapp and Watson, 1995) that the melted mafic source to the TTG magmas is partially hydrated. The remaining $\sim 80 \%$ component of the mafic protolith may consist of unaltered gabbros from structurally lower in the oceanic crust, beyond depths of hydrothermal alteration. Low $\delta^{18} \mathrm{O}$ pore-water within oceanic crust $\left(\delta^{18} \mathrm{O}=\right.$ from $0 \%$ to $-3 \%$ ) can also exist to several hundred meters (Perry et al., 1976) although such fluids are unlikely to be preserved at sufficient temperatures or pressures to be considered a feasible ${ }^{18} \mathrm{O}$-depleted component.

\subsection{Is oceanic crust the source of Eoarchaean TTG melts?}

Adakitic magmas are thought to be derived from highdegree slab melts and analogous to the Archaean TTG (Defant and Drummond, 1990; Martin, 1999). Bindeman et al. (2005) analysed the oxygen isotopic compositions of olivine phenocrysts from a suite of modern adakite lavas, obtaining calculated $\delta^{18} \mathrm{O}_{\mathrm{WR}}$ compositions ranging from $6.18 \%$ to $7.19 \%$ and averaging $6.60 \%$. Such compositions are only marginally higher than that of the primitive mantle and were interpreted to reflect the Si-rich compositions and low liquidus temperatures of the lavas, rather than to con- 


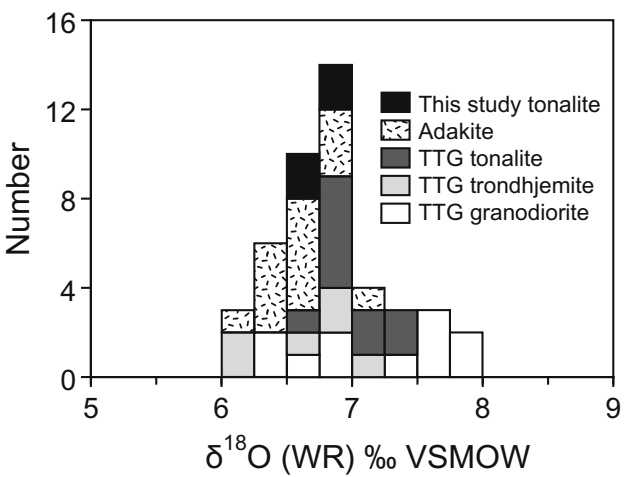

Fig. 9. Histogram of calculated $\delta^{18} \mathrm{O}_{\mathrm{WR}}$ compositions for IGC tonalites, modern adakites (Bindeman et al., 2005) and Archaean TTG rocks (King et al., 1998, 2000). Zircon-melt partition ratios for IGC and TTG magmas from Valley et al. (1994, 2005). $\mathrm{SiO}_{2}$ contents taken from Nutman et al. (1999, 2007b) and King et al. (1998, 2000). Olivine-melt fractionation for adakites is based on fractionation method one of Bindeman et al. (2005) with $57 \mathrm{wt} \%$ $\mathrm{SiO}_{2}$.

tamination by ${ }^{18} \mathrm{O}$-enriched sources. Calculated mean $\delta^{18} \mathrm{O}_{\mathrm{WR}}$ for the Greenland tonalite magmas $\mathrm{G} 01 / 36, \mathrm{G} 01 /$ 113 , G97/18 and 248228 ranges from $6.7 \%$ to $6.9 \%$ and lie within the range of modern adakites (Fig. 9), suggesting a similar origin. Calculated tonalite $\delta^{18} \mathrm{O}_{\mathrm{WR}}$ compositions from this study are also consistent with those of other Archaean TTG tonalites from the Superior Province (King et al., 1998, 2000; Fig. 9), while TTG trondhjemites and granodiorites from the same suites record a subtly broader range of compositions. While the appropriateness of associating the petrogenesis of Archaean TTG directly with that of Cenozoic adakites has been challenged (Smithies, 2000), these data support the observation of Bindeman et al. (2005) that based on the consistency of $\delta^{18} \mathrm{O}_{\mathrm{WR}}$ compositions, at least some Archaean TTG and modern adakite magmas may be generated by similar mechanisms, from broadly similar sources.

The $\mathrm{O}$ and $\mathrm{Hf}$ isotopic compositions of zircon from Archaean TTG magmas presented here could plausibly be generated by melting mafic crust in geological settings unrelated to convergent margins. Mid ocean ridge spreading environments record zircon $\delta^{18} \mathrm{O}$ compositions in equilibrium with mantle oxygen (Cavosie et al., 2005b) while a young upwelling asthenosphere could deliver a steady supply of material to the melting region. The limitation to this relatively low-pressure model is that melting would be unlikely to have taken place within the stability field of garnet. The melting of an overthickened oceanic plateau has also been suggested as a suitable scenario (White et al., 1999), however, accommodating the long-term chondritic Lu/Hf character of the reservoir may be problematic. The melting of an overthickened or floundering mafic underplate may also be considered (Petford and Atherton, 1996; Petford and Gallagher, 2001; Bolhar et al., 2008). However, mechanisms to hydrate the source material remain complicated. Hence, we favor the interpretation that the mafic protolith to the TTG magmas was shallowly subducted or buried oceanic crust.

Models for flat subduction of Archaean oceanic crust have been previously proposed as mechanisms to induce TTG magmatism (e.g. Smithies and Champion, 2000; Nutman et al., 2007a). Fig. 10 schematically illustrates an Eoarchaean convergent margin where young, hot, oceanic crust becomes shallowly subducted beneath a more mature arc crust. Imbricate stacking of multiple, oceanic crust layers would be more likely to introduce compositional heterogeneity and become jammed, while flat subduction would be less prone to such complications so is the mechanism

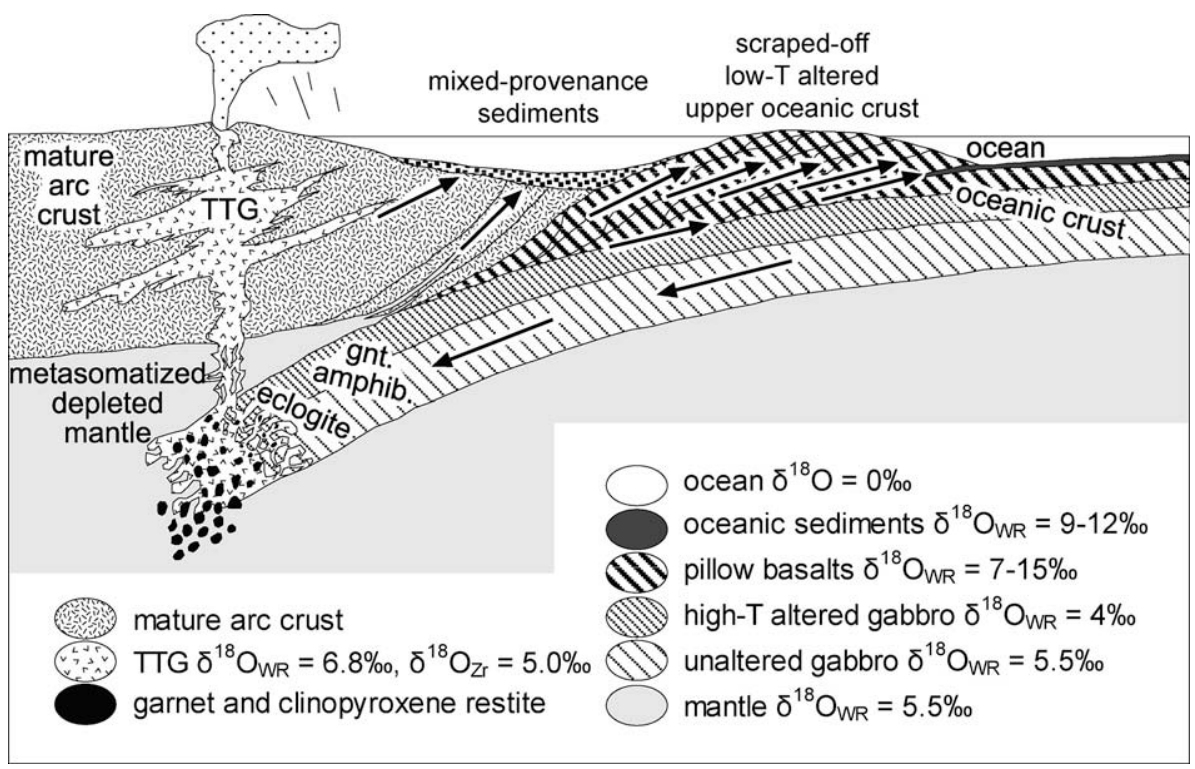

Fig. 10. Schematic diagram of key components involved in the flat subduction of Eoarchaean oceanic crust melting to produce early TTG magmas. 
explored in this discussion. ${ }^{18} \mathrm{O}$-enriched uppermost oceanic crustal sections are scraped off in an accretionary-wedgestyle thrust sequence that with the nearby felsic arc crust provides mixed-provenance sediments to adjacent basins. At the leading edge of an acute mantle wedge, thin slivers of upper mantle are caught up within the telescoping arc sequence. High-temperature altered and unaltered gabbros from deeper in the oceanic crustal section are transported further into the subduction system, converting to garnet amphibolite and eclogite prior to hydrous partial melting to form TTG melts. Magmas may ascend through a short section of metasomatised and depleted mantle before intruding the early continental crust to crystallise as tonalites or erupt as felsic volcanic lavas.

\subsection{Volcanic samples 248202 and 248203}

Because of the mantle-like $\mathrm{O}$ and $\mathrm{Hf}$ characteristics of the zircon in volcanic samples 248202 and 248203, their petrogenetic origin was probably similar to those of the TTG tonalites. Some of the bulk rock chemical features (e.g. severe $\mathrm{Na}_{2} \mathrm{O}$ depletion and carbonate addition) were imposed by post igneous crystallisation processes to which the generally chemically inert zircons were immune. These zircon signatures suggests felsic igneous protoliths that were altered either shortly after eruption most likely by weathering in the volcanic basin or less likely later during superimposed tectonothermal events, and do not reflect igneous assimilation of altered wall rocks.

\section{CONCLUSIONS}

${ }^{176} \mathrm{Hf} /{ }^{177} \mathrm{Hf}$ zircon analyses record initial mean compositions that are largely within error of CHUR estimates, indicating the long-term source of TTG magmas was characterized by chondritic Lu/Hf. Chondritic Lu/Hf sources to TTG melts over a 180 million year period suggest that processes of crustal growth operated incrementally during the Hadean and Eoarchaean. Either there was no formation of extensive continental crust in the early Hadean or continental crust was extensively recycled during the Earth's first billion years.

Olivine $\delta^{18} \mathrm{O}$ from a weakly-hydrated mantle-derived $\sim 3.8 \mathrm{Ga}$ harzburgite/dunite indicates continuity of the oxygen isotopic composition the Eoarchaean and modern mantle. Zircon from tonalitic and felsic volcanic magmas records mean $\delta^{18} \mathrm{O}$ compositions within error of zircon derived from the mantle or melts generated at similar temperatures, with no indication of disturbance to $\delta^{18} \mathrm{O}$ during late recrystallisation. Calculated whole rock $\delta^{18} \mathrm{O}$ compositions of tonalite melts are identical to those of modern adakites consistent with a similar origin. The mafic source that melted to produce TTG tonalite magmas may have contained up to $20 \%$ volumetric component of hydrothermally altered gabbro. Such mafic source rocks are widely found in the lower section of oceanic crustal sections. We postulate that elevated $\delta^{18} \mathrm{O}$ contaminants such as shallow oceanic crust and pelagic or continental sediments were scraped off the flatly subducted or buried mafic slab, and not ingested during TTG melting.

\section{ANALYTICAL METHODS}

\subsection{Grain mounting and imaging}

Zircon and olivine grains analysed here were taken from mineral separates extracted from rock samples collected for previous studies (see above). An exception was sample 492120, for which a new zircon separate was made specifically for this work. All zircon and olivine crystals were isolated from hand samples by clean crushing, heavy liquid and magnetic separation techniques. Approximately 100 grains from each sample were transferred onto double sided adhesive tape with a fine-tipped needle under a binocular microscope. Zircon was aligned with its c-axis horizontal, but olivine was positioned with random orientations. Zircon and olivine samples were mounted close to their respective reference materials, SL13 zircon, FC1 zircon, Temora-2 zircon and San Carlos olivine that were dispersed over the mount surface.

New generation SHRIMP megamounts were constructed to minimize geometric fractionation during $\mathrm{O}$ isotopic analysis (Ickert et al., 2008). The need for a different mount geometry precluded the use of the existing SHRIMP mounts for these samples and required that new $\mathrm{U}-\mathrm{Pb}$ analyses be undertaken for each sample in order that the age, $\mathrm{O}$ and $\mathrm{Hf}$ analyses were all from the same zircon domains. All grains were cast in epoxy and polished with a rotary polisher and $1 \mu \mathrm{m}$ diamond paste to expose crystal mid-sections. Prior to each $\mathrm{U}-\mathrm{Pb}$ or $\mathrm{O}$ analytical session, polished analytical surfaces were sequentially cleaned in an ultrasonic bath with petroleum spirit, ethanol, diluted laboratory detergent, $1 \mathrm{M} \mathrm{HCl}(1 \times$ quartz distilled $)$, and deionized $(18 \mathrm{M} \Omega) \mathrm{H}_{2} \mathrm{O}$ before being dried in a $60^{\circ} \mathrm{C}$ oven. A $100-120 \AA \mathrm{Au}$ or $\mathrm{Al}$ conductive layer was then evaporated onto the analytical surface and electronically checked for uniform and adequate conductivity before loading into the instrument. A $100 \AA \mathrm{Au}$ coat was used for all U-Pb analyses, and a $120 \AA \mathrm{Al}$ coat was used for subsequent $\mathrm{O}$ analyses.

Prior to $\mathrm{U}-\mathrm{Pb}$ analysis, the zircon was imaged with reflected light, transmitted light and SEM cathodoluminesence (CL) spectroscopy. This allowed identification of grain cracks, mineral inclusions and two-dimensional growth and recrystallisation textures to guide spot placement onto least-disturbed, oscillatory zoned, igneous growth domains. Following $\mathrm{U}-\mathrm{Pb}$ analysis, the zircons were again imaged with reflected light to record the precise location of the $\sim 2 \mu \mathrm{m}$ deep age determination sputtered pits to assist future beam-positioning. Mounts were then lightly re-polished, removing $\sim 5 \mu \mathrm{m}$ of zircon to expose a second 'fresh' surface for $\mathrm{O}$ isotopic analysis, free of topography from earlier pits, or extraneous $\mathrm{O}$ implanted by the $\mathrm{O}_{2}^{-}$primary beam during the earlier $\mathrm{U}-\mathrm{Pb}$ work (Benninghoven et al., 1987). Prior to O analysis this second analytical surface was imaged with CL to check for continuity of the zircon oscillatory growth zoning between the first and second surfaces. Following $\mathrm{O}$ analysis, the second analytical surface was re-imaged by an SEM in secondary electron mode to ensure that the $\sim 2 \mu \mathrm{m}$ deep, $\mathrm{O}$ analytical pits had been placed directly above the pits 
for age determination, that the profile of the $\mathrm{Cs}^{+}$primary beam had been well focused, and that the $\mathrm{O}$ analysis was performed on pristine zircon (Cavosie et al., 2005a). For Hf isotopic analysis by MC-ICPMS, the laser, which penetrates $\sim 50 \mu \mathrm{m}$ into the zircon, was subsequently centered directly over the pit formed during $\mathrm{O}$ analysis and within the same oscillatory growth domain. This method most reliably correlated the zircon $\mathrm{O}$ and $\mathrm{Hf}$ isotopic composition with a crystallisation age, given the limiting tradeoff between spatial resolution and analytical precision. An example of selected zircon imaging produced for a single grain is illustrated in Fig. 3. Olivine grains were simply imaged in reflected and transmitted light prior to $\mathrm{O}$ isotopic analysis.

\subsection{U-Pb geochronology with SHRIMP RG}

Zircon U-Pb ages were measured using the SHRIMP RG ion microprobe at the Research School of Earth Sciences (RSES), the Australian National University. The methods employed here are in standard use and described in detail by Stern (1998) and Williams (1998) and are summarized as follows. A 2-4 nA mass filtered $\mathrm{O}_{2}^{-}$primary beam was focused to a $\sim 30 \mu \mathrm{m}$ (long axis) elliptical spot and the beam rastered for $120 \mathrm{~s}$ to clean the mount surface prior to data acquisition. The magnet was stepped through peaks of ${ }^{90} \mathrm{Zr}_{2}{ }^{16} \mathrm{O},{ }^{204} \mathrm{~Pb},{ }^{206} \mathrm{~Pb},{ }^{207} \mathrm{~Pb},{ }^{208} \mathrm{~Pb},{ }^{238} \mathrm{U}$, ${ }^{232} \mathrm{Th}^{16} \mathrm{O}$ and ${ }^{238} \mathrm{U}^{16} \mathrm{O}$. FC1 zircon reference material was analysed once every three unknowns. Data were reduced using the Excel ${ }^{\mathrm{MM}}$ macro SQUID (Ludwig, 2001). Zircon reference materials SL13 (Claoué-Long et al., 1995; $\mathrm{U}=238 \mathrm{ppm}$ ) and FC1 (Paces and Miller, 1993; ${ }^{206} \mathrm{~Pb} /{ }^{238} \mathrm{U}$ age $=1099.0 \pm 0.5 \mathrm{Ma}$ ) were used for $\mathrm{U}$ abundance and ${ }^{206} \mathrm{~Pb} /{ }^{238} \mathrm{U}$ calibrations, respectively. Decay constants and the atomic ${ }^{238} \mathrm{U} /{ }^{235} \mathrm{U}$ ratio of 137.88 recommended by the IUGS Subcommission on Geochronology (Steiger and Jäger, 1977) were used to calculate ages. Corrections for common $\mathrm{Pb}$ were based on small amounts of measured ${ }^{204} \mathrm{~Pb}$ with isotopic compositions corresponding to a $\mathrm{Pb}$ growth model age of $3700 \mathrm{Ma}$ (Stacey and Kramers, 1975). Analytical uncertainties for individual spots are reported as $1 \sigma$ within-spot errors. From the six samples dated, a total of 142 spots yielded $\mathrm{U}-\mathrm{Pb}$ ages that were $>90 \%$ concordant. These areas on each grain were selected for further $\mathrm{O}$ and $\mathrm{Hf}$ isotopic analysis.

To calculate the igneous age for each sample as a single value, analyses were culled to selectively remove outliers. Outliers were statistically and/or geologically identified as younger $\mathrm{U}-\mathrm{Pb}$ ages that were products of local recrystallisation or $\mathrm{Pb}$-loss. The population of unrejected analyses from each sample was then pooled to produce crystallisation ages with the Excel ${ }^{\mathrm{TM}}$ macro Isoplot (Ludwig, 2003) in either of two ways. (1) In samples 248228, 248202 and 248203, weighted mean ages were calculated as the mean square of weighted deviates (MSWD's) for these samples was $<2.5$, (2.4, 0.7 and 1.5, respectively). Weighted mean 95\% confidence limit uncertainties for each respective sample were calculated from the inverse square of the assigned within-spot errors. (2) In samples G01/36, G01/113 and G97/18 where weighted mean MSWD's were $>2.5$, mean ages were instead calculated for each sample. Mean $1 \sigma$ uncertainties were calculated from one standard deviation of the population age. All weighted mean or mean ${ }^{207} \mathrm{~Pb} /{ }^{206} \mathrm{~Pb}$ ages are in agreement with earlier published geochronology on these samples.

\subsection{Oxygen isotopic analysis with SHRIMP II multi- collector}

Zircon and olivine oxygen isotopic compositions were determined using the SHRIMP II multi-collector ion microprobe at the RSES over 11 analytical sessions. A session for $\mathrm{O}$ isotopic analysis is defined as an uninterrupted period of data collection, with the same standard calibration. Sessions are separated by cold restarts, mount changes, interruptions to operation, or a major retuning of the instrument's primary or secondary beam. Instrumental conditions (Ickert et al., 2008) were typically set with a $3.5 \mathrm{nA}, 15 \mathrm{keV} \mathrm{Cs}^{+}$primary beam focused to an elliptical $30 \mu \mathrm{m}$ (long axis) spot, sampling $\sim 2 \mathrm{ng}$ of mineral per analysis. Surface charge was neutralized by a $45^{\circ}$ incident, broadly focused, moderate energy $(1.1 \mathrm{keV}) \mathrm{e}^{-}$beam, delivering $\sim 1 \mu \mathrm{A}$ of electrons from a Kimball Physics ELG-5 electron gun at a working distance of $20 \mathrm{~mm}$. The electron gun is mounted off the extraction lens housing and floated at primary column potential. The $10 \mathrm{kV}$ secondary extraction yields $\sim 320 \mathrm{pA}$ of secondary current, or $\sim 4.0 \times$ $10^{6}$ cps of ${ }^{18} \mathrm{O}$ and $\sim 2.0 \times 10^{9} \mathrm{cps}$ of ${ }^{16} \mathrm{O}$ on zircon. Isotopic ratios were produced by simultaneous measurement of ${ }^{18} \mathrm{O}^{-}$and ${ }^{16} \mathrm{O}^{-}$ions by dual Faraday cups with $10^{11} \Omega$ and $10^{10} \Omega$ resistors, respectively. Background counts of $\sim 3.5 \times 10^{3} \mathrm{cps}$ on ${ }^{18} \mathrm{O}$ and $\sim 1.2 \times 10^{4} \mathrm{cps}$ on ${ }^{16} \mathrm{O}$ were measured and subtracted during setup configuration. A $150 \mu \mathrm{m}$ source slit and $300 \mu \mathrm{m}$ collector slits limit beam truncation to $<5 \%$, providing a mass resolution of $\sim 2500$ at $1 \%$ peak height. This is sufficient to separate potential isobaric interferences on ${ }^{18} \mathrm{O}^{-}$from ${ }^{17} \mathrm{OH}^{-},{ }^{16} \mathrm{OD}^{-}$and ${ }^{16} \mathrm{OH}_{2}^{-}$. A $180 \mathrm{~s}$ pre-sputter and secondary auto-tuning in $z$ - and $y$-directions (horizontal and vertical along the beam line for extracted secondary ions) preceded ratio measurements. Data acquisition consisted of one set of 10 scans, each with $10 \mathrm{~s}$ integration times, leading to total count times of $\sim 100 \mathrm{~s}$ and complete analyses within approximately $5 \mathrm{~min}$. Within this time period within-spot precision, based on counting statistics for both samples and reference materials reached near theoretical limits of $\pm 0.3 \%(1 \sigma)$. Operating conditions were held constant during a single given session.

Each reference material's measured ${ }^{18} \mathrm{O} /{ }^{16} \mathrm{O}$ ratios, drift, within-spot and spot-to-spot precisions are summarized in Electronic Annex EA-3. Over the 11 analytical sessions, sample analyses were calibrated against 140, time integrated, bracketing analyses of reference materials $\mathrm{FC1}$ zircon (co-genetic with AS3 zircon, $\delta^{18} \mathrm{O}=5.34 \pm 0.03 \%$, ${ }^{18} \mathrm{O} /{ }^{16} \mathrm{O}=0.0020159$, Trail et al., 2007), Temora-2 zircon $\left(\delta^{18} \mathrm{O}=8.20 \pm 0.01 \%{ }^{\circ},{ }^{18} \mathrm{O} /{ }^{16} \mathrm{O}=0.0020216\right.$, Valley, 2003; Black et al., 2004) or matrix matched San Carlos olivine $\left(\mathrm{Mg} \#=90, \delta^{18} \mathrm{O}=5.35 \%,{ }^{18} \mathrm{O} /{ }^{16} \mathrm{O}=0.0020159\right.$, Norman et al., 2006; Eiler et al., 2007). All ${ }^{18} \mathrm{O} /{ }^{16} \mathrm{O}$ ratios are 
presented as $\delta^{18} \mathrm{O}$ notation, expressed as deviations from Vienna standard mean ocean water (VSMOW, ${ }^{18} \mathrm{O} /{ }^{16} \mathrm{O}=0.0020052$, Baertschi, 1976) in parts per thousand. Instrumental drift in all but two sessions was $<0.05 \%$ per analysis and corrected for using a linear fit. Electron-induced secondary ion emission (EISIE) was monitored before and after analysis, and found to provide a systematic and insignificantly minor contribution to the total secondary signal (typically $<10^{6} \mathrm{cps}$ of ${ }^{16} \mathrm{O}$ at analysis end). Spot-to-spot reproducibility of nominally homogeneous reference materials for a single session ranged from $\pm 1.0 \%$ to $\pm 0.3 \%$ o ( $1 \sigma$; Fig. $4 \mathrm{~A}$, Electronic Annex EA-3). Spot-to-spot precision was always worse than within-spot precision and was subsequently considered to be the best measure of precision for any given analysis. Analytical accuracy is indicated by the results from Temora- 2 functioning as a secondary reference material in sessions 4,5 , 6 and 7. Across all four sessions Temora-2 records a mean $\delta^{18} \mathrm{O}$ composition of $8.0 \pm 0.6 \%(1 \sigma)$ which lies within analytical error of the published value of $8.20 \pm 0.01 \%$ o $(1 \sigma$; Valley, 2003; Black et al., 2004).

Oxygen isotopic compositions for each sample correspond to grains with age determinations. For tonalite and felsic volcanic samples, weighted mean or mean compositions were calculated from the same zircon spots that were used for to provide pooled ${ }^{207} \mathrm{~Pb} /{ }^{206} \mathrm{~Pb}$ ages. For sample $\mathrm{G} 93 / 42$ all olivine analyses were included. For samples G01/36, G97/18, 248202, 248203 and G93/42 weighted mean calculations were made as MSWD's were all $<2.5$ (2.3, 0.6, 0.9, 0.6 and 1.4 , respectively). Weighted mean $95 \%$ confidence limit uncertainties were calculated from the inverse square of the assigned error from each analysis. For samples G01/113 and 248228 where weighted mean MSWD's were $>2.5$, mean ages were calculated with $1 \sigma$ uncertainties from one standard deviation of the pooled population.

\subsection{Zircon hafnium abundances with LA-ICPMS}

As the amount of oxygen isotope fractionation during ion-microprobe analysis can be matrix dependent (e.g. Peck et al., 2001), we determined $\mathrm{HfO}_{2}$ concentrations for three standard zircon reference materials and two selected samples to assess matrix variability. Following the acquisition of ${ }^{18} \mathrm{O} /{ }^{16} \mathrm{O}$ data on SHRIMP II, $\mathrm{HfO}_{2}$ concentrations were measured using the RSES Aligent 7500 ICPMS equipped with a Lamda Physik LPX 1201 UV ArF eximer laser and Ar-He flushed sample cell (Eggins et al., 1998). The laser was operated at $22 \mathrm{kV}$ with $120 \mathrm{~mJ}$ energy per pulse at $4 \mathrm{~Hz}$. Each acquisition consisted of a $20 \mathrm{~s}$ background followed by a $150 \mathrm{~s}$ collection period. Blocks of 10 unknowns were bracketed by analyses of NIST 612 glass reference material. Raw counts were converted to concentrations using "LABRAT 0.93" written for Lab VIEW by A. Kallio. Corrections for mass bias in the samples were made using NIST 612. Zircon $\mathrm{HfO}_{2}$ abundances were normalized to stoichiometric (32.77wt \%) $\mathrm{SiO}_{2}$. Mean $\mathrm{HfO}_{2}$ concentrations for FC1 $(1.2 \pm 0.2 \mathrm{wt} \%, 95 \%$ c.1., $n=18)$, Temora-2 $(1.0 \pm 0.1 \mathrm{wt} \%, 95 \% \quad$ c.l., $n=9)$ and 91500 $(0.6 \pm 0.1 \mathrm{wt} \%, 95 \%$ c.l., $n=5)$ are all in agreement with published values for these reference materials of
$1.20 \pm 0.11 \mathrm{wt}^{\mathrm{O}} \% \quad$ (Black et al., 2004), $0.98 \pm 0.01 \mathrm{wt} \%$ (Black et al., 2004) and $0.695 \mathrm{wt} \%$ (Wiedenbeck et al., 2004), respectively. Samples G01/36 (1.4 $\pm 0.1 \mathrm{wt} \%$, 95\% c.1., $n=3)$ and $\mathrm{G} 97 / 18(1.6 \pm 0.1 \mathrm{wt} \%, 95 \%$ c.1., $n=18)$ contain similar $\mathrm{HfO}_{2}$ abundances to reference materials (FC1 and Temora-2) demonstrating that corrections for variations in instrumental mass fractionation (IMF) resulting from large variations in Hf content (Eiler et al., 1997a) were not necessary. A more comprehensive test of the sensitivity of oxygen isotopic IMF to matrix effects in SHRIMP II was presented by Ickert et al. (2008).

\subsection{Hafnium isotopic analysis with LA-MC-ICPMS}

Zircon hafnium isotopic compositions were determined over three analytical sessions using the RSES ThermoFinnigan Neptune multi-collector ICPMS coupled to a ArF $\lambda=193 \mathrm{~nm}$ eximer 'HelEx' laser ablation system following methods described by Harrison et al. (2005). The laser was focused to a $47 \mu \mathrm{m}$ diameter circular spot firing at $5 \mathrm{~Hz}$ with an energy density at the sample surface of $\sim 10 \mathrm{~J} /$ $\mathrm{cm}^{2} .{ }^{171} \mathrm{Yb},{ }^{173} \mathrm{Yb},{ }^{174} \mathrm{Hf},{ }^{175} \mathrm{Lu},{ }^{176} \mathrm{Hf},{ }^{177} \mathrm{Hf},{ }^{178} \mathrm{Hf},{ }^{179} \mathrm{Hf}$ and ${ }^{181} \mathrm{Ta}$ isotopes were simultaneously measured in static-collection mode on 9 Faraday cups with $10^{11} \Omega$ resistors. Amplifier gains were calibrated at the start of each session. A large zircon crystal from the Monastery kimberlite was used to tune the mass spectrometer to optimum sensitivity. Analysis of a gas blank and a suite of secondary reference zircons (Monastery, Mud Tank, 91500, Temora-2 and FC1; Woodhead and Hergt, 2005) was systematically performed after every 10-12 samples. Data were acquired in $1 \mathrm{~s}$ integrations over $100 \mathrm{~s}$, but time slices were later cropped to periods maintaining steady ${ }^{176} \mathrm{Hf} /{ }^{177} \mathrm{Hf}$ signals during data reduction on a custom Excel ${ }^{\mathrm{TM}}$ spreadsheet written by S. Eggins. Total Hf signal intensity typically fell from 5 to $2 \mathrm{~V}$ during a single analysis.

The measured ${ }^{178} \mathrm{Hf} /{ }^{177} \mathrm{Hf},{ }^{174} \mathrm{Hf} /{ }^{177} \mathrm{Hf},{ }^{176} \mathrm{Lu} /{ }^{177} \mathrm{Hf}$ and ${ }^{176} \mathrm{Hf} /{ }^{177} \mathrm{Hf}$ ratios with $2 \sigma$ uncertainties for each of the 122 reference zircon analyses are presented in Electronic Annex EA-4. No corrections were applied to the data to normalize the measured ${ }^{176} \mathrm{Hf} /{ }^{177} \mathrm{Hf}$ ratios to published solution values. Mass bias was corrected using an exponential law (Russell et al., 1978; Chu et al., 2002; Woodhead et al., 2004) and a compositions for ${ }^{179} \mathrm{Hf} /{ }^{177} \mathrm{Hf}$ of 0.732500 (Patchett et al., 1981). As a quality check of this procedure ${ }^{178} \mathrm{Hf} /{ }^{177} \mathrm{Hf}$ ratios for all zircon reference materials and samples are reported $(n=244)$. A mean value of $1.467247 \pm 88(2 \sigma)$ lies within uncertainty of values published by Thirlwall and Anczkiewicz (2004).

$\mathrm{Yb}$ and Lu mass bias factors were assumed to be identical and normalized using an exponential correction to a ${ }^{173} \mathrm{Yb} /{ }^{171} \mathrm{Yb}$ ratio of 1.123456 (Thirlwall and Anczkiewicz, 2004). The intensity of the ${ }^{176} \mathrm{Hf}$ peak was accurately determined by removing isobaric interferences from ${ }^{176} \mathrm{Lu}$ and ${ }^{176} \mathrm{Yb}$. Interference-free ${ }^{175} \mathrm{Lu}$ and ${ }^{173} \mathrm{Yb}$ were measured and the interference peaks subtracted according to reported isotopic abundances of 0.02645 for ${ }^{176} \mathrm{Lu} /{ }^{175} \mathrm{Lu}$ and 0.786956 for ${ }^{176} \mathrm{Yb} /{ }^{173} \mathrm{Yb}$ (Thirlwall and Anczkiewicz, 2004). Owing to the substantial ${ }^{174} \mathrm{Yb}$ interference at mass $174,{ }^{174} \mathrm{Hf} /{ }^{177} \mathrm{Hf}$ ratios are also reported to demonstrate the 
effectiveness of the $\mathrm{Yb}$ interference correction procedure. An average ratio of $0.008653 \pm 85(2 \sigma)$ is in agreement with values published by Thirlwall and Anczkiewicz (2004).

Zircon ${ }^{176} \mathrm{Lu} /{ }^{177} \mathrm{Hf}$ ratios should be accurately determined by LA-MC-ICPMS to enable corrections for ingrowth of radiogenic ${ }^{176} \mathrm{Hf}$. Average measured ${ }^{176} \mathrm{Lu} /{ }^{177} \mathrm{Hf}$ ratios within reference zircon (Monastery, 0.000013; Mud Tank, 0.000051; 91500, 0.000362; Temora-2, 0.001041; FC1, 0.001043; Electronic Annex EA-4) are in good agreement with the solution values reported by Woodhead and Hergt (2005) of $0.000009,0.000042,0.000311,0.001090$ and 0.001262 , respectively. The range of ${ }^{176} \mathrm{Lu} /{ }^{177} \mathrm{Hf}$ measured in the reference zircons brackets the mean ${ }^{176} \mathrm{Lu} /{ }^{177} \mathrm{Hf}$ ratios from samples G01/36 (0.000459), G01/ 113 (0.000537), G97/18 (0.000449), 248228 (0.000755), $248202(0.000765)$ and $248203(0.000805)$.

The mean ${ }^{176} \mathrm{Hf} /{ }^{177} \mathrm{Hf}$ ratios for the five reference zircons (Monastery: 0.282737 \pm 36 ; Mud Tank: 0.282511 \pm 36 ; 91500: $\quad 0.282312 \pm 77$; Temora-2: $0.282674 \pm 70 ; \quad \mathrm{FC} 1$ : $0.282157 \pm 55,2 \sigma)$ deviate from published solution values of Woodhead and Hergt (2005) by $0.0,0.1,0.2,-0.4$ and $-1.0 \varepsilon_{\mathrm{Hf}}$ units, respectively (Fig. 4B, Electronic Annex EA4). The mean of all ${ }^{176} \mathrm{Hf} /{ }^{177} \mathrm{Hf}$ analyses for each reference zircon lies within $2 \sigma$ uncertainty of their respective solution value. No correlation exists between ${ }^{176} \mathrm{Hf} /{ }^{177} \mathrm{Hf}$ and ${ }^{178} \mathrm{Hf} /{ }^{177} \mathrm{Hf},{ }^{174} \mathrm{Hf} /{ }^{177} \mathrm{Hf}$ or ${ }^{176} \mathrm{Lu} /{ }^{177} \mathrm{Hf}$ ratios for any zircon reference materials, including high $\mathrm{Lu} / \mathrm{Hf}$ Temora-2 and FC1 (Electronic Annex EA-5). This indicates that calculations for mass bias and $\mathrm{Yb}$ interference corrections were accurately applied. The -1 epsilon unit discrepancy between measured and published ${ }^{176} \mathrm{Hf} /{ }^{177} \mathrm{Hf}$ ratios in $\mathrm{FC1}$ reference material may in part relate to the variability in ${ }^{176} \mathrm{Hf} /{ }^{177} \mathrm{Hf}$ solution analyses reported by Woodhead and Hergt (2005). That is, there is likely real variation in the Hf isotopic composition of this reference zircon population. Low Lu/Hf reference materials Monastery and Mud Tank zircon $\left({ }^{176} \mathrm{Lu} /{ }^{177} \mathrm{Hf} \sim 0.00003\right)$ average 0.0 and $0.1 \varepsilon_{\mathrm{Hf}}$ units offset, respectively, while 91500 with ${ }^{176} \mathrm{Lu} /{ }^{177} \mathrm{Hf}$ ratios $\sim 0.0003$, most similar to those of the zircon samples analysed in this study $\left({ }^{176} \mathrm{Lu} /{ }^{177} \mathrm{Hf}=0.0004-0.0008\right)$ averages $\varepsilon_{\mathrm{Hf}}=0.2$.

For the unknown zircons, initial ${ }^{176} \mathrm{Hf} /{ }^{177} \mathrm{Hf}$ ratios for each spot were calculated using their individual SHRIMP measured ${ }^{207} \mathrm{~Pb} /{ }^{206} \mathrm{~Pb}$ ages, present day CHUR compositions of ${ }^{176} \mathrm{Hf} /{ }^{177} \mathrm{Hf}=0.282785 \pm 11, \quad{ }^{176} \mathrm{Lu} /{ }^{177} \mathrm{Hf}=$ $0.0336 \pm 1$ (Bouvier et al., 2008), and a $\lambda^{176} \mathrm{Lu}$ decay constant of $1.867 \pm 8 \times 10^{-11} \mathrm{yr}^{-1}$ (Scherer et al., 2001; Söderlund et al., 2004). For zircons from each rock, weighted mean initial Hf isotopic compositions were also calculated and correspond with their igneous crystallisation age and oxygen isotopic composition, in that all were measured from the same zircon domains. Again only analyses that were used for earlier weighted mean or mean age determinations were included. For all samples weighted mean calculations were made as MSWD's were all $<1$. Within-spot uncertainties for each analysis are typically from \pm 1.0 to $\pm 2.0 \varepsilon_{\mathrm{Hf}}$ units at the $2 \sigma$ level. Several sources of uncorrelated error may exist within these LA-MC-ICPMS analyses that do not account for the external scatter seen in some reference zircons (e.g. 91500). Therefore, a conservative approach is taken to estimate the absolute uncertainty of each spot that is used to calculate weighted mean $\varepsilon_{\mathrm{Hf}}$ compositions. Within-spot errors for individual analyses are summed in quadrature with an estimate of external reproducibility from the zircon reference materials. This is taken to be $\pm 1.9 \varepsilon_{\mathrm{Hf}}$ units, based on the average external reproducibility of all five reference materials over all session (Monastery $= \pm 1.3 \varepsilon_{\mathrm{Hf}}$, Mud Tank $=$ $\pm 1.3 \varepsilon_{\mathrm{Hf}}, \quad 91500= \pm 2.7 \varepsilon_{\mathrm{Hf}}, \quad$ Temora- $= \pm 2.5 \varepsilon_{\mathrm{Hf}}, \quad \mathrm{FC} 1=$ $1.9 \varepsilon_{\mathrm{Hf}}$; (Fig. 4B, Electronic Annex EA-4). Within-spot errors are quoted in the text, and figures, while the inverse square of assigned absolute errors are used to calculate the weighted mean ( $\pm 95 \%$ c.l.) compositions for each sample.

\section{ACKNOWLEDGMENTS}

We thank Jeff Vervoort, Tony Kemp and one anonymous reviewer for their constructive reviews that improved this manuscript and the patient editorial handling of Alan Brandon. Samples were collected during field work funded by ANU: G93/42, G97/18, G01/36 and G01/113; or by Geological Survey of Denmark and Greenland (GEUS) and the NATO advanced studies institute: 248202, 248203, 248228 and 492120. GEUS is thanked for permission to publish data on these samples. We thank Bud Baadsgaard for supplying zircon separates of 248202, 248203 and 248228. All analytical work was supported by the Australian Research Council Grants DP0342798 and DP0342794 and was undertaken while Hiess was a Ph.D. student at ANU supported by APA and Jaeger scholarships. We thank Shane Paxton and Jon Mya for zircon separations; Ryan Ickert and Peter Holden for contributions to SHRIMP O analysis development; Malcolm McCulloch for access to the Neptune; Les Kinsley for assistance with running LA-MCICPMS; Steve Eggins for providing a template for Hf data reduction; Chuck McGee for technical assistance with LA-ICPMS analysis; Antti Kallio for providing LABRAT software; Yuri Amelin, Bob Rapp, Joerg Herman and Trevor Ireland for helpful discussions.

\section{APPENDIX A. SUPPLEMENTARY DATA}

Supplementary data associated with this article can be found, in the online version, at doi:10.1016/ j.gca.2009.04.019.

\section{REFERENCES}

Albarède F., Scherer E. E., Blichert-Toft J., Rosing M., Simionovici A. and Bizzarro M. (2006) $\Gamma$-Ray irradiation in the early Solar System and the conundrum of the ${ }^{176} \mathrm{Lu}$ decay constant. Geochim. Cosmochim. Acta 70, 1261-1270.

Alt J. C., Muehlenbachs K. and Honnorez J. (1986) An oxygen isotopic profile through the upper kilometer of the oceanic crust, DSDP Hole 504B. Earth Planet. Sci. Lett. 80, 217-229.

Amelin Y. (2005) Meteorite phosphates show constant ${ }^{176} \mathrm{Lu}$ decay rate since 4557 million years ago. Science 310, 839-841.

Amelin Y. and Davis W. J. (2005) Geochemical test for branching decay of ${ }^{176}$ Lu. Geochim. Cosmochim. Acta 69, 465-473.

Amelin Y., Lee D.-C., Halliday A. N. and Pidgeon R. T. (1999) Nature of the Earth's earliest crust from hafnium isotopes in single detrital zircons. Nature 399, 252-255.

Amelin Y., Lee D. C. and Halliday A. N. (2000) Early-middle Archaean crustal evolution deduced from $\mathrm{Lu}-\mathrm{Hf}$ and $\mathrm{U}-\mathrm{Pb}$ isotopic studies of single zircon grains. Geochim. Cosmochim. Acta 64, 4205-4225. 
Atherton M. P. and Petford N. (1993) Generation of sodium-rich magmas from newly underplated basaltic crust. Nature $\mathbf{3 6 2}$, 144-146.

Baadsgaard H. (1983) U/Pb isotope systematics on minerals from the gneiss complex at Isukasia, West Greenland. Rapp. Grønlands Geol. Unders. 112, 35-42.

Baadsgaard H., Nutman A. P., Bridgwater D., McGregor V. R., Rosing M. T. and Allaart J. H. (1984) The zircon geochronology of the Akilia association and the Isua supracrustal belt, West Greenland. Earth Planet. Sci. Lett. 68, 221-228.

Baadsgaard H., Nutman A. P., Rosing M. T., Bridgwater D. and Longstaffe F. J. (1986) Alteration and metamorphism of Amitsoq gneisses from the Isukasia area, West Greenland: recommendations for isotope studies of the early crust. Geochim. Cosmochim. Acta 50, 2165-2172.

Baertschi P. (1976) Absolute ${ }^{18} \mathrm{O}$ content of standard mean ocean water. Earth Planet. Sci. Lett. 31, 341-344.

Barker F. and Arth J. G. (1976) Generation of trondhjemitictonalitic liquids and Archean bimodal trondhjemite-basalt suites. Geology 4, 596-600.

Bennett V. (2003) Compositional evolution of the mantle. Treat. Geochem. 2, 493-519.

Bennett V. C., Nutman A. P. and McCulloch M. T. (1993) Nd isotopic evidence for transient, highly depleted mantle reservoirs in the early history of the Earth. Earth Planet. Sci. Lett. 119, 299-317.

Bennett V. C., Nutman A. P. and Esat T. M. (2002) Constraints on mantle evolution from ${ }^{187} \mathrm{Os} /{ }^{188} \mathrm{Os}$ isotopic compositions of Archean ultramafic rocks from southern West Greenland $(3.8 \mathrm{Ga})$ and Western Australia $(3.46 \mathrm{Ga})$. Geochim. Cosmochim. Acta 66, 2615-2630.

Bennett V. C., Brandon A. D. and Nutman A. P. (2007) Coupled ${ }^{142} \mathrm{Nd}-{ }^{143} \mathrm{Nd}$ isotopic evidence for Hadean mantle dynamics. Science 318, 1907-1910.

Benninghoven A., Rüdenauer F. G. and Werner H. W. (1987) Secondary Ion Mass Spectrometry: Basic Concepts, Instrumental Aspects, Applications and Trends. Wiley, Hoboken, NJ.

Bindeman I. N., Eiler J. M., Yogodzinski G. M., Tatsumi Y., Stern C. R., Grove T. L., Portnyagin M., Hoernle K. and Danyushevsky L. V. (2005) Oxygen isotope evidence for slab melting in modern and ancient subduction zones. Earth Planet. Sci. Lett. 235, 480-496.

Bizzarro M., Baker J. A., Haack H., Ulfbeck D. and Rosing M. (2003) Early history of Earth's crust-mantle system inferred from hafnium isotopes in chondrites. Nature 421, 931-933.

Black L. P., Kamo S. L., Allen C. M., Davis D. W., Aleinikoff J. N., Valley J. W., Mundil R., Campbell I. H., Korsch R. J., Williams I. S. and Foudoulis C. (2004) Improved ${ }^{206} \mathrm{~Pb} /{ }^{238} \mathrm{U}$ microprobe geochronology by the monitoring of a traceelement-related matrix effect; SHRIMP, ID-TIMS, ELA-ICPMS and oxygen isotope documentation for a series of zircon standards. Chem. Geol. 205, 115-140.

Blichert-Toft J. and Albarède F. (2008) Hafnium isotopes in Jack Hills zircons and the formation of the Hadean crust. Earth Planet. Sci. Lett. 265, 686-702.

Blichert-Toft J., Chauvel C. and Albarède F. (1997) Separation of $\mathrm{Hf}$ and $\mathrm{Lu}$ for high-precision isotope analysis of rock samples by magnetic sector-multiple collector ICP-MS. Contrib. Mineral. Petrol. 127, 248-260.

Bolhar R., Kamber B. S., Moorbath S., Whitehouse M. J. and Collerson K. D. (2005) Chemical characterization of Earth's most ancient clastic metasediments from the Isua Greenstone Belt, southern West Greenland. Geochim. Cosmochim. Acta 69, 1555-1573.

Bolhar R., Weaver S. D., Whitehouse M. J., Palin J. M., Woodhead J. D. and Cole J. W. (2008) Sources and evolution of arc magmas inferred from coupled $\mathrm{O}$ and $\mathrm{Hf}$ isotope systematics of plutonic zircons from the Cretaceous Separation Point Suite (New Zealand). Earth Planet. Sci. Lett. 268, 312-324.

Bouvier A., Vervoort J. D. and Patchett J. (2008) The Lu-Hf and $\mathrm{Sm}-\mathrm{Nd}$ isotopic composition of CHUR: constraints from unequilibrated chondrites and implications for the bulk composition of the terrestrial planets. Earth Planet. Sci. Lett. doi:10.1016/j.epsl.2008.06.010.

Caro G., Bourdon B., Birck J.-L. and Moorbath S. (2003) ${ }^{146} \mathrm{Sm}-{ }^{142} \mathrm{Nd}$ evidence from Isua metamorphosed sediments for early differentiation of the Earth's mantle. Nature 423, 428-432.

Caro G., Bourdon B., Birck J.-L. and Moorbath S. (2006) Highprecision ${ }^{142} \mathrm{Nd} /{ }^{144} \mathrm{Nd}$ measurements in terrestrial rocks: constraints on the early differentiation of the Earth's mantle. Geochim. Cosmochim. Acta 70, 164-191.

Cates N. L. and Mojzsis S. J. (2006) Chemical and isotopic evidence for widespread Eoarchean metasedimentary enclaves in southern West Greenland. Geochim. Cosmochim. Acta 70, $4229-4257$.

Cavosie A. J., Valley J. W., Wilde S. A. and E.I.M.F. (2005a) Magmatic $\delta^{18} \mathrm{O}$ in $4400-3900 \mathrm{Ma}$ detrital zircons: a record of the alteration and recycling of crust in the early Archean. Earth Planet. Sci. Lett. 235, 663-681.

Cavosie A. J., Kita N. T. and Valley J. W. (2005b) Magmatic $\delta^{18} \mathrm{O}$ in zircons from gabbros and serpentinized peridotite at the Mid-Atlantic Ridge (ODP leg 153). Eos Trans. AGU. Fall Meet. Suppl., San Francisco. \#OS33A-1453 (abstract).

Chauvel C. and Blichert-Toft J. (2001) A hafnium isotope and trace element perspective on melting of the depleted mantle. Earth Planet. Sci. Lett. 190, 137-151.

Choi S. H., Mukasa S. B., Andronikov A. V., Osanai Y., Harley S. L. and Kelly N. M. (2006) Lu-Hf systematics of the ultra-high temperature Napier Metamorphic Complex in Antarctica: evidence for the early Archean differentiation of Earth's mantle. Earth Planet. Sci. Lett. 246, 305-316.

Chu N. C., Taylor R. N., Chavagnac V., Nesbitt R. W., Boella R. M., Milton J. A., German C. R., Bayon G. and Burton K. (2002) Hf isotope ratio analysis using multi-collector inductively coupled plasma mass spectrometry: an evaluation of isobaric interference corrections. J. Anal. Atom. Spectrom. 17, $1567-1574$.

Claoué-Long J. C., Compston W., Roberts J. and Fanning C. M. (1995) Two Carboniferous ages: a comparison of SHRIMP zircon dating with conventional zircon ages and ${ }^{40} \mathrm{Ar} /{ }^{39} \mathrm{Ar}$ analysis. In Geochronology, Time Scales and Global Stratigraphic Correlation, vol. 54 (eds. W. A. Berggren, D. V. Kent, M. P. Aubry and J. Hardenbol), pp. 3-21. Geochronology, Time Scales and Global Stratigraphic Correlation. SEPM Special Publication.

Compston W., Kinny P. D., Williams I. S. and Foster J. J. (1986) The age and $\mathrm{Pb}$ loss behaviour of zircons from the Isua supracrustal belt as determined by ion microprobe. Earth Planet. Sci. Lett. 80, 71-81.

Condie K. C. (1981) Archean Greenstone Belts. Elsevier, Amsterdam.

Criss R. E. and Taylor H. R. J. (1986) Meteoric-hydrothermal systems. In Stable Isotopes in High Temperature Geological Processes, vol. 16 (eds. J. W. Valley, H. P. J. Taylor and O. N. J. R). Reviews in Mineralogy, pp. 373-424.

Defant M. J. and Drummond M. S. (1990) Derivation of some modern arc magmas by melting of young subducted lithosphere. Nature 347, 662-665.

Drummond M. S. and Defant M. J. (1990) A model for trondhjemite-tonalite-dacite genesis and crustal growth via slab melting: Archaean to modern comparisons. J. Geophys. Res. 95(B13), 21503-21521. 
Dymek R. F., Brothers S. C. and Schiffries C. M. (1988) Petrogenesis of ultramafic metamorphic rocks from the 3800 Ma Isua Supracrustal Belt, West Greenland. J. Petrol. 29, 1353-1397.

Eggins S. M., Kinsley L. P. J. and Shelley J. M. G. (1998) Deposition and element fractionation processes during atmospheric pressure laser sampling for analysis by ICP-MS. Appl. Surf. Sci. 127-129, 278-286.

Eiler J. M. (2001) Oxygen isotope variations of basaltic lavas and upper mantle rocks. In Stable Isotope Geochemistry, vol. 43 (eds. J. W. Valley and D. R. Cole). Reviews in Mineralogy and Geochemistry, pp. 319-364.

Eiler J. M., Farley K. A., Valley J. W., Stolper E. M., Hauri E. H. and Craig H. (1995) Oxygen isotope evidence against bulk recycled sediment in the mantle sources of Pitcairn Island lavas. Nature 377, 138-141.

Eiler J. M., Graham C. and Valley J. W. (1997a) SIMS analysis of oxygen isotopes: matrix effects in complex minerals and glasses. Chem. Geol. 138, 221-244.

Eiler J. M., Farley K. A., Valley J. W., Hauri E., Craig H., Hart S. R. and Stolper E. M. (1997b) Oxygen isotope variations in ocean island basalt phenocrysts. Geochim. Cosmochim. Acta 61, 2281-2293.

Eiler J. M., Schiano P., Valley J. W., Kita N. T. and Stolper E. M. (2007) Oxygen-isotope and trace element constraints on the origins of silica-rich melts in the subarc mantle. Geochem. Geophys. Geosyst. 8, Q09012. doi:10.1029/2006GC001503.

Frei R. and Polat A. (2007) Source heterogeneity for the major components of $3.7 \mathrm{Ga}$ Banded Iron Formations (Isua Greenstone Belt, Western Greenland): Tracing the nature of interacting water masses in BIF formation. Earth Planet. Sci. Lett. 253, 266-281.

Friend C. R. L. and Nutman A. P. (2005) Complex 3670-3500 Ma orogenic episodes superimposed on juvenile crust accreted between 3850 and $3690 \mathrm{Ma}$, Itsaq Gneiss Complex, southern West Greenland. J. Geol. 113, 375-397.

Friend C. R. L., Nutman A. P. and McGregor V. R. (1987) LateArchaean tectonics in the Faeringehavn - Tre Brodre area, south of Buksefjorden, southern West Greenland. J. Geol. Soc. Lond. 144, 369-376.

Friend C. R. L., Bennett V. C. and Nutman A. P. (2002) Abyssal peridotites $>3800 \mathrm{Ma}$ from southern West Greenland; field relationships, petrography, geochronology, whole-rock and mineral chemistry of dunite and harzburgite inclusions in the Itsaq Gneiss Complex. Contrib. Mineral. Petrol. 143, 71-92.

Furnes H., de Wit M., Staudigel H., Rosing M. and Muehlenbachs K. (2007) A vestige of Earth's oldest ophiolite. Science 315, 1704-1707.

Gill J. B. (1981) Orogenic Andesites and Plate Tectonics. Springer, Berlin.

Gregory R. T. and Taylor H. P. (1981) An oxygen isotope profile in a section of Cretaceous oceanic crust, Samail Ophiolite, Oman: evidence for $\delta^{18} \mathrm{O}$ buffering of the oceans by deep $(>5 \mathrm{~km})$ seawater-hydrothermal circulation at mid-ocean ridges. $J$. Geophys. Res. 86(B4), 2737-2755.

Griffin W. L., McGregor V. R., Nutman A., Taylor P. N. and Bridgwater D. (1980) Early Archaean granulite-facies metamorphism south of Ameralik, West Greenland. Earth Planet. Sci. Lett. 50, 59-74.

Griffin W. L., Belousova E. A., Shee S. R., Pearson N. J. and O'Reilly S. Y. (2004) Archean crustal evolution in the northern Yilgarn Craton: U-Pb and Hf-isotope evidence from detrital zircons. Precamb. Res. 131, 231-282.

Harrison T. M., Blichert-Toft J., Muller W., Albarède F., Holden P. and Mojzsis S. J. (2005) Heterogeneous Hadean hafnium: evidence of continental crust at 4.4-4.5 Ga. Science 310, 19471950.
Harrison T. M., Schmitt A. K., McCulloch Malcolm T. and Lovera O. M. (2008) Early $(\geqslant 4.5 \mathrm{Ga})$ formation of terrestrial crust: $\mathrm{Lu}-\mathrm{Hf}, \delta^{18} \mathrm{O}$, and Ti thermometry results for Hadean zircons. Earth Planet. Sci. Lett. 268, 476-486.

Hiess J., Nutman A. P., Bennett V. C. and Holden P. (2008) Ti-inzircon thermometry applied to contrasting Archean metamorphic and igneous systems. Chem. Geol. 247, 323-338.

Honda M., Nutman A. P. and Bennett V. C. (2003) Xenon compositions of magmatic zircons in 3.64 and 3.81 Ga metagranitoids from Greenland; a search for extinct ${ }^{244} \mathrm{Pu}$ in ancient terrestrial rocks. Earth Planet. Sci. Lett. 207, 69-82.

Hoskin P. W. O. and Schaltegger U. (2003) The composition of zircon and igneous and metamorphic petrogenesis. In Zircon, vol. 53 (eds. J. M. Hanchar and P. W. O. Hoskin). Reviews in Mineralogy and Geochemistry, pp. 27-62.

Ickert R. B., Hiess J., Williams I. S., Holden P., Ireland T. R., Chappell B. W., Lanc P., Schram N., Foster J. J. and Clement S. W. (2008) Determining high precision, in situ, oxygen isotope ratios with a SHRIMP II: analyses of MPI- DING silicate-glass reference materials and zircon from contrasting granites. Chem. Geol. 257, 114-128.

Iizuka T. and Hirata T. (2005) Improvements of precision and accuracy in in situ $\mathrm{Hf}$ isotope microanalysis of zircon using the laser ablation-MC-ICPMS technique. Chem. Geol. 220, 121137.

Jahn B. M., Glikson A. Y., Peucat J. J. and Hickman A. H. (1981) REE geochemistry and Isotopic data of Archean silicic volcanics and granitoids from the Pilbara Block, Western Australia: implications for the early crustal evolution. Geochim. Cosmochim. Acta 45, 1633-1652.

Kamber B. S., Ewart A., Collerson K. D., Bruce M. C. and McDonald G. D. (2002) Fluid-mobile trace element constraints on the role of slab melting and implications for Archaean crustal growth models. Contrib. Mineral. Petrol. 144, 38-56.

Kasting J. F., Howard M. T., Wallmann K., Veizer J., Shields G. and Jaffrés J. (2006) Paleoclimates, ocean depth, and the oxygen isotopic composition of seawater. Earth Planet. Sci. Lett. 252, $82-93$.

Kay R. W. (1978) Aleutian magnesian andesites; melts from subducted Pacific Ocean crust. J. Volcanol. Geotherm. Res. 4, 117-132.

Kemp A. I. S., Hawkesworth C. J., Paterson B. A. and Kinny P. D. (2006) Episodic growth of the Gondwana supercontinent from hafnium and oxygen isotopes in zircon. Nature 439, 580-583.

Kemp A. I. S., Hawkesworth C. J., Foster G. L., Paterson B. A., Woodhead J. D., Hergt J. M., Gray C. M. and Whitehouse M. J. (2007) History of granitic rocks from $\mathrm{Hf}-\mathrm{O}$ isotopes in zircon. Science 315, 980-983.

Kemp A. I. S., Foster G. L., Scherstén A., Whitehouse M. J., Darling J. and Storey C. (2009) Concurrent $\mathrm{Pb}-\mathrm{Hf}$ isotope analysis of zircon by laser ablation multi-collector ICP-MS, with implications for the crustal evolution of Greenland and the Himalayas. Chem. Geol. 261, 244-260.

King E. M. (2001) Oxygen isotope study of magmatic source and alteration of granitic rocks in the Western United States and Superior Province, Canada, Ph.D. thesis, University of Wisconsin.

King E. M., Valley J. W., Davis D. W. and Edwards G. R. (1998) Oxygen isotope ratios of Archean plutonic zircons from granite-greenstone belts of the Superior Province: indicator of magmatic source. Precamb. Res. 92, 365-387.

King E. M., Valley J. W. and Davis D. W. (2000) Oxygen isotope evolution of volcanic rocks at the Sturgeon Lake volcanic complex, Ontario. Can. J. Earth Sci. 37, 39-50.

Kinny P. D. and Maas R. (2003) Lu-Hf and Sm-Nd isotope systems in zircon. In Zircon, vol. 53 (eds. J. M. Hanchar and P. 
W. O. Hoskin). Reviews in Mineralogy and Geochemistry, pp. 327-341.

Knauth L. P. and Lowe D. R. (2003) High Archean climatic temperature inferred from oxygen isotope geochemistry of cherts in the $3.5 \mathrm{Ga}$ Swaziland Supergroup, South Africa. Geol. Soc. Am. Bull. 115, 566-580.

Kolodny Y. and Epstein S. (1976) Stable isotope geochemistry of deep sea cherts. Geochim. Cosmochim. Acta 40, 1195-1209.

Longstaffe F. J. and Schwarcz H. P. (1977) ${ }^{18} \mathrm{O} /{ }^{16} \mathrm{O}$ of Archean clastic metasedimentary rocks: a petrogenetic indicator for Archean gneisses? Geochim. Cosmochim. Acta 41, 1303-1312.

Lowe D. R. (1994) Archean greenstone-related sedimentary rocks. In Archean Crustal Evolution (ed. K. C. Condie), pp. 121-169.

Lowry D., Appel P. W. U. and Rollinson H. R. (2003) Oxygen isotopes of an early Archaean layered ultramafic body, southern West Greenland: implications for magma source and postintrusion history. Precamb. Res. 126, 273-288.

Ludwig K. R. (2001) Squid 1.02 User's Manual, Berkley Geochronology Centre, Special Publication No. 2, Rev. June 20, 19p.

Ludwig K. R. (2003) Isoplot 3.00 User's Manual: A Geochronological Toolkit for Microsoft Excel, Berkeley Geochronological Center, Special Publication No. 4, Rev. May 30, 70p.

Martin H. (1986) Effect of steeper Archean geothermal gradient on geochemistry of subduction-zone magmas. Geology 14, 753-756.

Martin H. (1987) Petrogenesis of Archaean trondhjemites, tonalites and granodiorites from eastern Finland; major and trace element geochemistry. J. Petrol. 28, 921-953.

Martin H. (1999) Adakitic magmas: modern analogues of Archaean granitoids. Lithos 46, 411-429.

Martin H., Chauvel C. and Jahn B. M. (1983) Major and trace element geochemistry and crustal evolution of Archaean granodioritic rocks from eastern Finland. Precamb. Res. 21, 159-180.

Martin H., Smithies R. H., Rapp R., Moyen J.-F. and Champion D. (2005) An overview of adakite, tonalite-trondhjemitegranodiorite (TTG), and sanukitoid; relationships and some implications for crustal evolution. Lithos 79, 1-24.

Mattey D., Lowry D. and Macpherson C. (1994) Oxygen isotope composition of mantle peridotite. Earth Planet. Sci. Lett. 128, 231-241.

McGregor V. R. (1973) The early Precambrian gneisses of the Godthaab district, West Greenland. Philos. Trans. R. Soc. Lond. A 273, 343-358.

Moorbath S., O'Nions R. K., Pankhurst R. J., Gale N. H. and McGregor V. R. (1972) Further rubidium-strontium age determinations on the very early Precambrian rocks of Godthåb district: West Greenland. Nat. Phys. Sci. 240, 78-82.

Moyen J. F. and Stevens G. (2006) Experimental constraints on TTG petrogenesis: implications for Archean geodynamics. In Archean Geodynamics and Environments, vol. 164 (eds. K. Benn, J.-C. Mareschal and K. C. Condie). American Geophysical Union.

Muehlenbachs K. (1986) Alteration of the oceanic crust and the ${ }^{18} \mathrm{O}$ history of seawater. In Stable Isotopes in High Temperature Geological Processes, vol. 16 (eds. J. W. Valley, H. P. Taylor and J. R. O'Neil). Reviews in Mineralogy, pp. 425-444.

Muehlenbachs K. (1998) The oxygen isotopic composition of the oceans, sediments and the seafloor. Chem. Geol. 145, 263273.

Norman M. D., McCulloch M. T., O’Neill H. St. C. and Yaxley G. M. (2006) Magnesium isotopic analysis of olivine by laserablation multi-collector ICP-MS: composition dependent matrix effects and a comparison of the Earth and Moon. $J$. Anal. At. Apectrom. 21, 50-54.

Nutman A. P. and Bridgwater D. (1986) Early Archaean Amitsoq tonalites and granites of the Isukasia area, southern West Greenland; development of the oldest-known sial. Contrib. Mineral. Petrol. 94, 137-148.
Nutman A. P. and Friend C. R. L. (2007) Adjacent terranes with ca. 2715 and $2650 \mathrm{Ma}$ high-pressure metamorphic assemblages in the Nuuk region of the North Atlantic Craton, southern West Greenland: Complexities of Neoarchaean collisional orogeny. Precamb. Res. 155, 159-203.

Nutman A. P. and Hiess J. (2009) A granitic inclusion suite within igneous zircons from a $3.81 \mathrm{Ga}$ tonalite (W. Greenland): implications for Hadean crustal evolution studies using detrital zircons. Chem. Geol. 261, 76-81.

Nutman A. P., Allaart J. H., Bridgwater D., Dimroth E. and Rosing M. (1984) Stratigraphic and geochemical evidence for the depositional environment of the early Archaean Isua Supracrustal Belt, southern West Greenland. Precamb. Res. 25, 365-396.

Nutman A. P., McGregor V. R., Friend C. R. L., Bennett V. C. and Kinny P. D. (1996) The Itsaq Gneiss Complex of southern West Greenland; the world's most extensive record of early crustal evolution (3900-3600 Ma). Precamb. Res. 78, 1-39.

Nutman A. P., Bennett V. C., Friend C. R. L. and Rosing M. T. (1997) $\sim 3710$ and $\geqslant 3790 \mathrm{Ma}$ volcanic sequences in the Isua (Greenland) supracrustal belt; structural and $\mathrm{Nd}$ isotope implications. Chem. Geol. 141, 271-287.

Nutman A. P., Bennett V. C., Friend C. R. L. and Norman M. D. (1999) Meta-igneous (non-gneissic) tonalites, quartzdiorites from an extensive ca. $3800 \mathrm{Ma}$ terrain south of the Isua supracrustal belt, southern West Greenland; constraints on early crust formation. Contrib. Mineral. Petrol. 137, 364-388.

Nutman A. P., Bennett V. C., Friend C. R. L. and McGregor V. R. (2000) The early Archaean Itsaq Gneiss Complex of southern West Greenland; the importance of field observations in interpreting age and isotopic constraints for early terrestrial evolution. Geochim. Cosmochim. Acta 64, 3035-3060.

Nutman A. P., Friend C. R. L. and Bennett, Jr., V. C. (2002) Evidence for 3650-3600 Ma assembly of the northern end of the Itsaq gneiss complex, Greenland: implication for early Archaean tectonics. Tectonics 21, 1-28.

Nutman A. P., Friend C. R. L., Horie K. and Hidaka H. (2007a) The Itsaq Gneiss Complex of southern West Greenland and the construction of Eoarchaean crust at convergent plate boundaries. In Earth's Oldest Rocks (eds. M. J. van Kranendonk, R. H. Smithies and V. C. Bennett). Elsevier, Amsterdam, pp. 187-218.

Nutman A. P., Bennett V. C., Friend C. R. L., Horie K. and Hidaka H. (2007b) $\sim 3850$ Ma tonalites in the Nuuk region, Greenland: geochemistry and their reworking within an Eoarchaean gneiss complex. Contrib. Mineral. Petrol. 154, 385-408.

Paces J. B. and Miller J. D. (1993) Precise U-Pb age of Duluth Complex and related mafic intrusions, northeastern Minnesota: Geochronological insights to physical, petrogenetic, paleomagnetic, and tectonomagnetic processes associated with the $1.1 \mathrm{Ga}$ midcontinent rift system. J. Geophys. Res. 98(B8), 13997-14013.

Patchett P. J. and Tatsumoto M. (1980a) A routine high-precision method for Lu-Hf isotope geochemistry and chronology. Contrib. Mineral. Petrol. 75, 263-267.

Patchett P. J. and Tatsumoto M. (1980b) Lu-Hf total-rock isochron for the eucrite meteorites. Nature 288, 571-574.

Patchett P. J., Kouvo O., Hedge C. E. and Tatsumoto M. (1981) Evolution of continental crust and mantle heterogeneity: evidence from Hf isotopes. Contrib. Mineral. Petrol. 78, 279-297.

Peck W. H., Valley J. W., Wilde S. A. and Graham C. M. (2001) Oxygen isotope ratios and rare earth elements in $3.3-4.4 \mathrm{Ga}$ zircons: ion microprobe evidence for high $\delta^{18} \mathrm{O}$ continental crust and oceans in the Early Archean. Geochim. Cosmochim. Acta 65, 4215-4229.

Perry E. A., Gieskes J. M. and Lawrence J. R. (1976) Mg, Ca and ${ }^{18} \mathrm{O} /{ }^{16} \mathrm{O}$ exchange in the sediment-pore water system, Hole 149 , DSDP. Geochim. Cosmochim. Acta 40, 413-423. 
Petford N. and Atherton M. (1996) Na-rich partial melts from newly underplated basaltic crust: the Cordillera Blanca Batholoith. Peru. J. Petrol. 37, 1491-1521.

Petford N. and Gallagher K. (2001) Partial melting of mafic (amphibolitic) lower crust by periodic influx of basaltic magma. Earth Planet. Sci. Lett. 193, 483-499.

Pettingill H. S. and Patchett P. J. (1981) Lu-Hf total-rock age for the Amitsoq gneisses, West Greenland. Earth Planet. Sci. Lett. 55, 150-156.

Polat A. and Hofmann A. W. (2003) Alteration and geochemical patterns in the 3.7-3.8 Ga Isua greenstone belt, West Greenland. Precamb. Res. 126, 197-218.

Polat A., Hofmann A. W. and Rosing M. T. (2002) Boninitelike volcanic rocks in the $3.7-3.8 \mathrm{Ga}$ Isua greenstone belt, West Greenland: geochemical evidence for intra-oceanic subduction zone processes in the early Earth. Chem. Geol. 184, 231-254.

Rapp R. P. and Watson E. B. (1995) Dehydration Melting of Metabasalt at 8-32-kbar - implications for Continental Growth and Crust-Mantle Recycling. J. Petrol. 36, 891931.

Rapp R. P., Watson E. B. and Miller C. F. (1991) Partial melting of amphibolite/eclogite and the origin of Archean trondhjemites and tonalites. Precamb. Res. 51, 1-25.

Rapp R. P., Shimizu N. and Norman M. D. (2003) Growth of early continental crust by partial melting of eclogite. Nature $\mathbf{4 2 5}$, 605-609.

Rollinson H. (2007) Recognising early Archaean mantle: a reappraisal. Contrib. Mineral. Petrol. 154, 241-252.

Russell W. A., Papanastassiou D. A. and Tombrello T. A. (1978) $\mathrm{Ca}$ isotope fractionation on the Earth and other solar system material. Geochim. Cosmochim. Acta 42, 1075-1090.

Ryder G. (2002) Mass flux in the ancient Earth-Moon system and benign implications for the origin of life on Earth. J. Geophys. Res. 107(E4), 5022. doi:10.1029/2001JE001583.

Scherer E., Munker C. and Mezger K. (2001) Calibration of the lutetium-hafnium clock. Science 293, 683-687.

Sguigna A. P., Larabee A. J. and Waddington J. C. (1982) The half-life of ${ }^{176} \mathrm{Lu}$ by a $\gamma-\gamma$ coincidence measurement. Can. J. Phys. 60, 361-364.

Smithies R. H. (2000) The Archaean tonalite-trondhjemite-granodiorite (TTG) series is not an analogue of Cenozoic adakite. Earth Planet. Sci. Lett. 182, 115-125.

Smithies R. H. and Champion D. C. (2000) The Archaean high-Mg diorite suite: links to tonalite-trondhjemite-granodiorite magmatism and implications for early Archaean crustal growth. $J$. Petrol. 41, 1653-1671.

Söderlund U., Patchett P. J., Vervoort J. D. and Isachsen C. E. (2004) The ${ }^{176}$ Lu decay constant determined by Lu-Hf and U$\mathrm{Pb}$ isotope systematics of Precambrian mafic intrusions. Earth Planet. Sci. Lett. 219, 311-324.

Stacey J. S. and Kramers J. D. (1975) Approximation of terrestrial lead isotope evolution by a two-stage model. Earth Planet. Sci. Lett. 26, 207-221.

Staudigel H., Davies G. R., Hart S. R., Marchant K. M. and Smith B. M. (1995) Large scale isotopic $\mathrm{Sr}, \mathrm{Nd}$ and $\mathrm{O}$ isotopic anatomy of altered oceanic crust: DSDP/ODP sites 417/418. Earth Planet. Sci. Lett. 130, 169-185.

Steiger R. H. and Jäger E. (1977) Subcommission on geochronology: convention on the use of decay constants in geo- and cosmochronology. Earth Planet. Sci. Lett. 36, 359-362.

Stern R. A. (1998) High-resolution SIMS determination of radiogenic trace-isotope ratios in minerals. In Modern Approaches to Ore and Environmental Mineralogy, vol. 27 (eds. L. J. Cabri and D. J. Vaughan). Mineralogical Association of Canada Short Course Series, pp. 41-268.
Taylor S. R. and McLennan S. M. (1995) The geochemical evolution of the continental crust. Rev. Geophys. 33, 241265.

Tera F., Papanastassiou D. A. and Wasserburg G. J. (1974) Isotopic evidence for a terminal lunar cataclysm. Earth Planet. Sci. Lett. 22, 1-21.

Thirlwall M. F. and Anczkiewicz R. (2004) Multidynamic isotope ratio analysis using $\mathrm{MC}-\mathrm{ICP}-\mathrm{MS}$ and the causes of secular drift in $\mathrm{Hf}, \mathrm{Nd}$ and $\mathrm{Pb}$ isotope ratios. Int. J. Mass Spectrom. 235, 59-81.

Trail D., Mojzsis S. J., Harrison T. M., Schmitt A. K., Watson E. B. and Young E. D. (2007) Constraints on Hadean zircon protoliths from oxygen isotopes, Ti-thermometry, and rare earth elements. Geochem. Geophys. Geosyst. 8, Q06014. doi:10.1029/2006GC001449.

Valley J. W. (2003) Oxygen isotopes in zircon. In Zircon, vol. 53 (eds. J. M. Hanchar and P. W. O. Hoskin). Reviews in Mineralogy and Geochemistry, pp. 343-385.

Valley J. W., Chiarenzelli J. R. and McLelland J. M. (1994) Oxygen isotope geochemistry of zircon. Earth Planet. Sci. Lett. 126, 187-206.

Valley J. W., Kinny P. D., Schulze D. J. and Spicuzza M. J. (1998) Zircon megacrysts from kimberlite: oxygen isotope variability among mantle melts. Contrib. Mineral. Petrol. 133, 1-11.

Valley J. W., Bindeman I. N. and Peck W. H. (2003) Empirical calibration of oxygen isotope fractionation in zircon. Geochim. Cosmochim. Acta 67, 3257-3266.

Valley J., Lackey J., Cavosie A., Clechenko C., Spicuzza M., Basei M., Bindeman I., Ferreira V., Sial A., King E., Peck W., Sinha A. and Wei C. (2005) 4.4 billion years of crustal maturation: oxygen isotope ratios of magmatic zircon. Contrib. Mineral. Petrol. 150, 561-580.

Veizer J. and Mackenzie F. T. (2003) Evolution of sedimentary rocks. Treat. Geochem. 7, 369-407.

Vervoort J. D. and Blichert-Toft J. (1999) Evolution of the depleted mantle: Hf isotope evidence from juvenile rocks through time. Geochim. Cosmochim. Acta 63, 533-556.

Vervoort J. D. and Patchett J. P. (1996) Behavior of hafnium and neodymium isotopes in the crust: constraints from Precambrian crustally derived granites. Geochim. Cosmochim. Acta 60, 37173733.

Vervoort J. D., Patchett P. J., Gehrels G. E. and Nutman A. P. (1996) Constraints on early Earth differentiation from hafnium and neodymium isotopes. Nature 379, 624-627.

Walker J. C. G. and Lohmann K. C. (1989) Why the oxygen isotopic composition of seawater changes with time? Geophys. Res. Lett. 16, 323-326.

Wallmann K. (2001) The geological water cycle and the evolution of marine $\delta^{18} \mathrm{O}$ values. Geochim. Cosmochim. Acta 65, 24692485.

White R. V., Tarney J., Kerr A. C., Saunders A. D., Kempton P. D., Pringle M. S. and Klaver G. T. (1999) Modification of an oceanic plateau, Aruba, Dutch Caribbean: implications for the generation of continental crust. Lithos 46, 43-68.

Whitehouse M. J. and Kamber B. S. (2002) On the overabundance of light rare earth elements in terrestrial zircons and its implication for Earth's earliest magmatic differentiation. Earth Planet. Sci. Lett. 204, 333-346.

Whitehouse M. J., Kamber B. S. and Moorbath S. (1999) Age significance of $\mathrm{U}-\mathrm{Th}-\mathrm{Pb}$ zircon data from early Archaean rocks of West Greenland - a reassessment based on combined ion-microprobe and imaging studies. Chem. Geol. 160, 201-224.

Wiedenbeck M., Hanchar J. M., Peck W. H., Sylvester P., Valley J., Whitehouse M., Kronz A., Morishita Y., Nasdala L., Fiebig J., Franchi I., Girard J. P., Greenwood R. C., 
Hinton, Kita N., Mason P. R. D., Norman M., Ogasawara M., Piccoli P. M., Rhede D., Satoh H., Schulz-Dobrick B., Skår Ø., Spicuzza M. J., Terada K., Tindle A., Togashi S., Vennemann T., Xie Q. and Zheng Y.-F. () Further characterization of the zircon 91500 crystal. Geostand. Geoanal. Res. 28, 9-39.

Wilde S. A., Valley J. W., Peck W. H. and Graham C. M. (2001) Evidence from detrital zircons for the existence of continental crust and oceans on the Earth $4.4 \mathrm{Gyr}$ ago. Nature 409, 175-178.

Williams I. S. (1998) U-Th-Pb geochronology by ion microprobe. Rev. Econ. Geol. 7, 1-35.

Woodhead J. and Hergt J. (2005) A preliminary appraisal of seven natural zircon reference materials for in situ $\mathrm{Hf}$ isotope determination. Geostand. Geoanal. Res. 29, 183-195.
Woodhead J., Hergt J., Shelley M., Eggins S. and Kemp R. (2004) Zircon Hf-isotope analysis with an excimer laser, depth profiling, ablation of complex geometries, and concomitant age estimation. Chem. Geol. 209, 121-135.

Workman R. K. and Hert S. R. (2005) Major and trace element composition of the depleted MORB mantle (DMM). Earth Planet. Sci. Lett. 231, 53-72.

Xu J.-F., Shinjo R., Defant M. J., Wang Q. and Rapp R. P. (2002) Origin of Mesozoic adakitic intrusive rocks in the Ningzhen area of East China: partial melting of delaminated lower continental crust? Geology 30, 1111-1114.

Associate editor: Alan D. Brandon 